Collection SFN 10 (2010) 379-423

(C) Owned by the authors, published by EDP Sciences, 2010

DOI: $10.1051 / \mathrm{sfn} / 2010006$

\title{
Diffusion inélastique de neutrons par temps de vol
}

\author{
J. Ollivier ${ }^{1}$ et J.-M. Zanotti ${ }^{2}$ \\ ${ }^{1}$ Institut Laue-Langevin, 6 rue J. Horowitz, 38042 Grenoble Cedex, France \\ ${ }^{2}$ Laboratoire Léon Brillouin (CEA-CNRS), CEA Saclay, 91191 Gif-sur-Yvette Cedex, France
}

\begin{abstract}
Résumé. Des événements dynamiques microscopiques contrôlent de nombreux processus physiques qui régissent les propriétés de la matière condensée: transport, magnétisme, catalyse et même, fonction d'objets biologiques. La diffusion inélastique des neutrons est une sonde irremplaçable de ces phénomènes. Elle donne accès aux mouvements atomiques et moléculaires sur des distances de quelques angstroems à quelques dizaines de nanomètres, sur des échelles temporelles de quelques dixièmes de picoseconde à la microseconde. Dans ce cadre, les différentes techniques de diffusion inélastique de neutrons par temps de vol occupent une position centrale. Après avoir introduit les principes et les éléments clés d'une mesure en temps de vol, cet article présente les principales classes de spectromètres et détaille leurs caractéristiques. Un accent particulier est mis sur les forces, les faiblesses et les complémentarités des différentes technologies.
\end{abstract}

\section{INTRODUCTION}

Une mesure par temps de vol consiste à déterminer l'énergie cinétique d'une particule, en évaluant sa vitesse i.e. le temps mis pour parcourir une distance connue. Cette méthode, d'autant plus robuste qu'elle est simple, au moins dans son principe, a marqué l'histoire de la Physique. En 1849, H. Fizeau [1,2] a estimé la vitesse de la lumière, avec une précision remarquable, en mesurant le temps de parcours entre Suresnes et Montmartre d'un faisceau lumineux pulsé par une roue dentée. Seulement trois ans après la découverte du neutron par Chadwick en 1932, c'est également une mesure par temps de vol qui a permis à Dunning et al. de [3] de montrer que conformément aux prédictions de E. Fermi, l'énergie d'un faisceau de neutrons après modération par de la paraffine, se situait dans une gamme d'énergie, dite thermique, bien plus faible que l'énergie cinétique initiale des neutrons produits lors du processus de fission nucléaire.

Déjà, l'élément clé de cet ancêtre des spectromètres à neutrons à temps de vol était un système de choppers (de l'anglais to chop: hacher), constitué d'une paire de disques en aluminium distants de quelques dizaines de centimètres, couverts de secteurs de cadmium, et entrainés par un arbre commun ${ }^{1}$. Après la seconde guerre mondiale, cette technique a été mise à profit pour déterminer l'énergie des neutrons de fission émis par les premiers réacteurs nucléaires expérimentaux. Fermi, et ses collaborateurs avaient innové, notamment en développant un collimateur tournant. Ce chopper de Fermi [5] consistait en un empilement vertical de feuilles d'aluminium et de cadmium, en rotation dans le faisceau. Une fois les réacteurs mis au point, ce dispositif fut utilisé pour étudier les modérateurs : graphite puis eau légère et eau lourde.

C'est dans les années 1950 que les aspects fondamentaux des processus dynamiques dans la matière condensée ont, à la fois, utilisé et motivé le développement des techniques basées sur la diffraction des mono-cristaux (deux-axes et trois-axes) et/ou sur les mesures par temps de vol. Cependant

\footnotetext{
${ }^{1}$ On utilise le fait que l'aluminium est transparent aux neutrons alors que le cadmium est un puissant absorbant. L'alternance dans le faisceau des parties transparentes et absorbantes permet de produire des bouffées de neutrons. Un chopper moderne est présenté sur la figure 11.

This is an Open Access article distributed under the terms of the Creative Commons Attribution-Noncommercial License 3.0, which permits unrestricted use, distribution, and reproduction in any noncommercial medium, provided the original work is properly cited.
} 
une électronique performante manquait encore pour (i) assurer un contrôle suffisamment fin de la rotation et du phasage des choppers, (ii) pour l'acquisition et (iii) le traitement du grand volume de données inhérent à ce type de mesure [4]. Après une première tentative de mise au point d'un système couplant deux choppers de Fermi, par P. Egelstaff à Harwell en 1956, les premiers instruments réellement exploitables ne sont apparus que dans les années 1960. Dans le même temps, grâce aux premiers spectromètres trois-axes construits à Chalk-River (Canada) en 1955, B.N. Brockhouse ${ }^{2}$ jetait les bases de la spectroscopie inélastique par diffusion de neutrons telle que nous la connaissons aujourd'hui.

\section{LE TEMPS DE VOL : POUR QUELLE PHYSIQUE ?}

\subsection{Petit rappel de physique}

Dans le cadre de la dualité onde-corpuscule, à un neutron d'énergie $E=\frac{1}{2} m_{n} \cdot v^{2}$, est associée une onde de longueur d'onde :

$$
\lambda=\frac{h}{m_{n} \cdot v}
$$

où $h=6.62 .10^{-34} \mathrm{~J} . \mathrm{s}, m_{n}=1.67 .10^{-27} \mathrm{~kg}$ et $v$, sont la constante de Planck, la masse et la vitesse du neutron ${ }^{3,4}$. Ainsi l'énergie d'un neutron de quelques angstrœms de longueur d'onde est-elle de quelques meV. C'est cette double adéquation entre à la fois (i) la longueur d'onde/l'énergie d'un neutron et (ii) l'ordre de grandeur des distances interatomiques/l'énergie des excitations dans la matière condensée, qui confère à la diffusion de neutrons son statut de technique spectroscopique de premier ordre. L'interaction du faisceau incident avec la matière conduit à un processus de diffusion ${ }^{5}$ dont résulte un échange de moment $\vec{Q}$ et d'énergie $\hbar \omega$ entre les neutrons incidents et l'échantillon. Le facteur de structure dynamique ${ }^{6}, S(\vec{Q}, \omega)$, qui décrit la dépendance angulaire et la distribution en énergie de l'intensité diffusée permet alors d'accéder, simultanément, à la structure et à la dynamique de l'échantillon. On montre que cette grandeur est reliée à la probabilité de trouver un atome de l'échantillon à une position $\vec{r}^{\prime}$, à un temps t, sachant que ce même atome (ou un autre) était à $\vec{r}$ à $\mathrm{t}=0$.

\subsection{Phénomènes accessibles par diffusion de neutrons}

La technique de temps de vol permet l'étude de phénomènes dynamiques d'origines nucléaires ou magnétiques (Fig. 1). On distingue la zone quasi-élastique qui est caractéristique des phénomènes de relaxation et diffusion. Centrée autour du pic élastique, elle peut s'étendre sur plusieurs dizaines de meV, par exemple sur des relaxations de spins, contrairement à ce que laisse supposer son nom. Au delà ce cette gamme en énergie, les signaux correspondent à l'échange de quanta d'énergie entre les neutrons

\footnotetext{
2 Co-lauréat, avec C.G. Shull, du prix Nobel de physique 1994 pour ses travaux menant à l'élaboration des techniques de diffusion des neutrons utilisées dans l'étude de la matière.

3 On en déduit la relation de base: $\tau[\mu \mathrm{s}]=C . \lambda[\AA]$. $D[\mathrm{~m}]$ où $\mathrm{C}=m_{n} / h=252.8 \mu \mathrm{s} . \mathrm{m}^{-1} \AA^{-1}$ qui permet de relier la longueur d'onde $\lambda$ et le temps de vol $\tau$ sur la distance $D$.

${ }^{4}$ L'énergie d'un neutron de longueur d'onde $\lambda[\AA]$ est $E[m e V]=\frac{1}{2} \frac{h^{2}}{m_{n}} \frac{1}{\lambda^{2}}=\frac{81.805}{\lambda^{2}}=11.605 \mathrm{~T}[\mathrm{~K}]$. Ainsi, un neutron de 1.8 $\AA$ porte une énergie de $25 \mathrm{meV}=200 \mathrm{~cm}^{-1}=300 \mathrm{~K}$ i.e. l'énergie thermique à température ambiante (d'où la dénomination "neutron thermique").

5 Il s'agit d'une interaction quantique dont nous pouvons, ici, faire abstraction pour ne prendre en compte que sa conséquence: les noyaux cibles se comportent comme des sources ponctuelles secondaires de neutrons.

${ }^{6}$ En toute rigueur, on devrait écrire $S(\vec{Q}, \hbar \omega)$. Mais un usage malheureux, auquel nous nous plions nous aussi, veut que dans le facteur de structure dynamique, l'énergie soit désignée par $\omega$ et non pas $\hbar \omega$.
} 


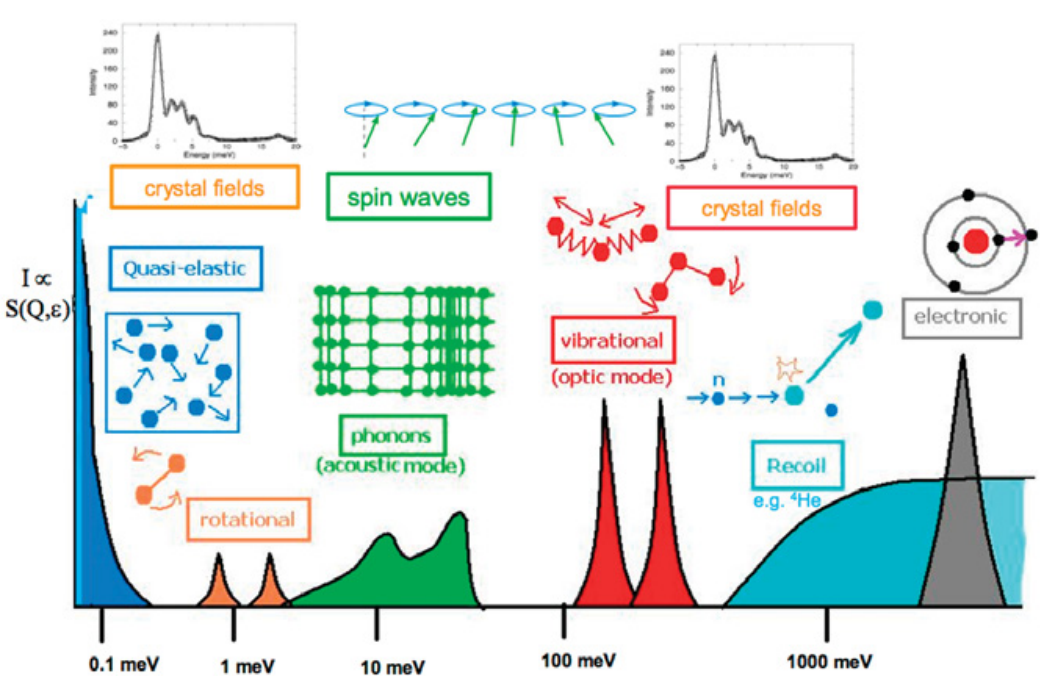

Figure 1. Schématisation des facteurs de structure dynamique, $S(Q, \omega)$, d'une sélection de phénomènes accessibles par diffusion inélastique de neutrons (d'après [8]).

et l'échantillon. Cette gamme de transfert d'énergie est souvent qualifiée de spectroscopique (neutron spectroscopy).

\subsection{Gammes spatiales et temporelles sondées par temps de vol}

Sa relative simplicité de mise en oeuvre et sa robustesse rendent la technique de temps de vol très pertinente pour mesurer la distribution en énergie d'un faisceau polychromatique de neutrons. Elle permet de couvrir[9] les gammes des neutrons épithermiques, chauds (quelques centaines de meV), thermiques $(25 \mathrm{meV}, 300 \mathrm{~K})$ et froids (quelques $\mathrm{meV}$ ) couramment utilisées pour l'étude de la matière condensée (voir Tableau 2), mais également le domaine des très hautes énergies. Ce dernier domaine dépasse le cadre de cet ouvrage, mais à titre d'exemple, on peut citer le spectromètre n_ToF du CERN (Genève) qui permet d'analyser des énergies de $1 \mathrm{eV}$ à $250 \mathrm{MeV}$ grâce à une base de temps de vol de $185 \mathrm{~m}$ [6]. Des spectromètres neutrons à temps de vol sont également utilisés sur les réacteurs à fusion nucléaire pour déterminer, le ratio deutérium/tritium dans le plasma en fusion (voir par exemple [7]).

La figure 2 précise le champ d'action des spectromètres à temps de vol dans le paysage instrumental en neutronique. Ils permettent de couvrir la gamme étendue de l'espace $(Q, \omega)$, intermédiaire entre les instruments à filtres $(3$ axes $)$ et les instruments à haute résolution comme les spectromètres à rétrodiffusion (backscattering) ou à écho de spin. Cette représentation fait cependant abstraction des temps de comptage nécessaires à la mesure d'un signal exploitable et de certaines limitations techniques: la quantité d'échantillon et/ou le faible pouvoir diffusant, peuvent, par exemple, être un obstacle à une mesure en rétrodiffusion; en spin-echo l'absence de polarisation - induite par un rapport défavorable des diffusions cohérente et incohérente - peut rendre la mesure de la dynamique du système impossible dans certains domaines de vecteur de diffusion.

La gamme temporelle sondée par temps de vol fait appel à une notion instrumentale fondamentale dont il sera abondamment question par la suite : la résolution spatiale et la résolution en énergie. En effet, dans la pratique, les grandeurs de distances et de temps sur lesquelles est basée la mesure sont définies à une incertitude près. Une fonction d'appareil résulte de ces imperfections. Elle limite non seulement la précision de la mesure mais aussi les gammes de temps accessibles (cf. section 3.3). 


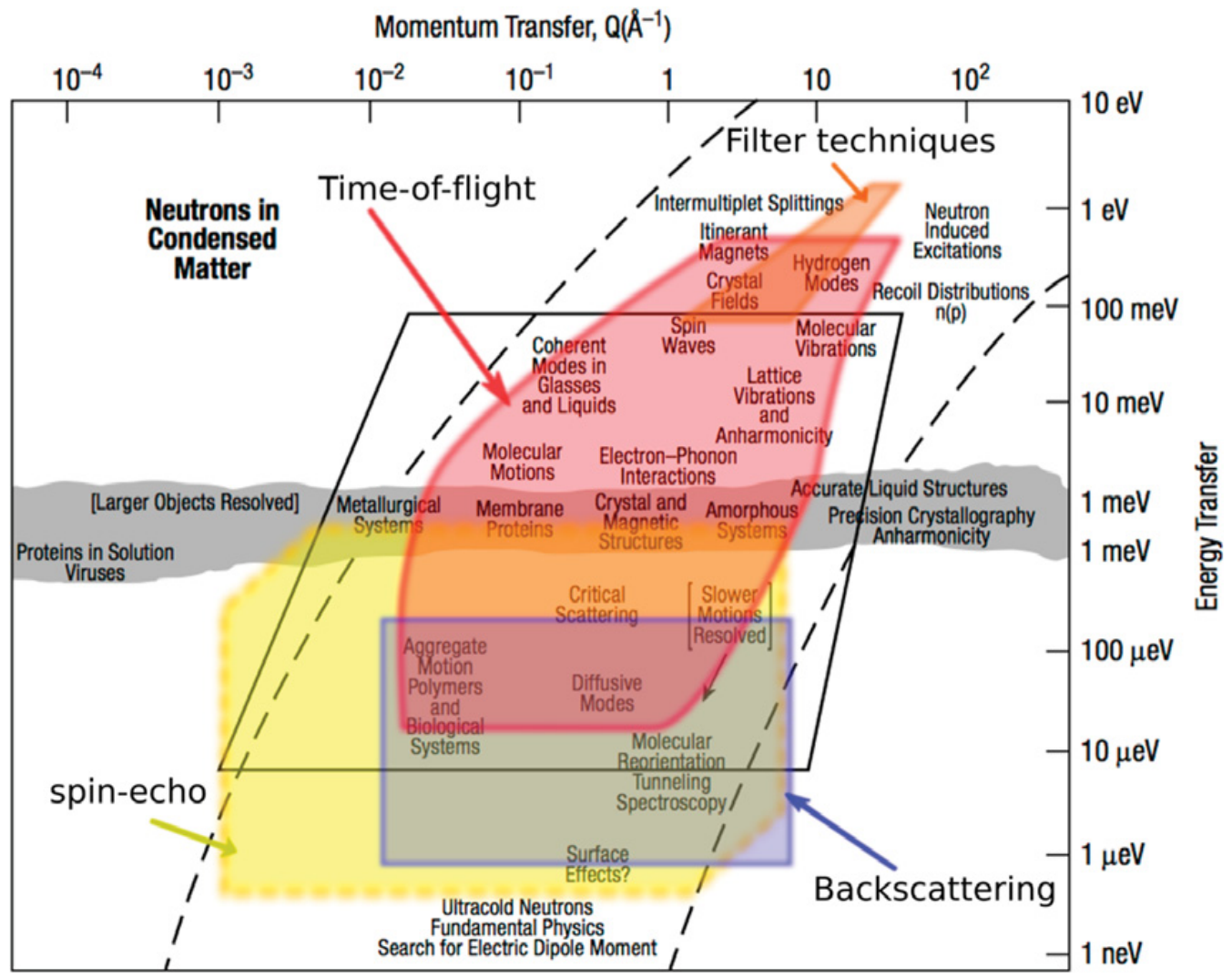

Figure 2. Echelles spatiales $\left(r[\AA] \approx \frac{2 \pi}{Q\left[^{-1}\right]}\right)$ et temporelles $(t[\mathrm{ps}] \approx 0.658 / \hbar \omega[\mathrm{meV}])$ associées aux principaux phenomènes physiques potentiellement observables par diffusion inélastique de neutrons. Les régions de l'espace $(Q, \omega)$ accessibles par les principales familles d'instruments (techniques à filtres, 3 axes, temps de vol, rétrodiffusion et spin-echo) sont précisées (d'après R. Pynn, LANSC).

\section{PRINCIPE D'UNE MESURE PAR TEMPS DE VOL}

La figure 3 décrit le principe d'une expérience de diffusion inélastique de neutrons par temps de vol. Les neutrons incidents d'un faisceau monochromatique d'énergie :

$$
E_{i}=\frac{\hbar^{2} k_{i}^{2}}{2 m_{n}}, k_{i}=\frac{2 \pi}{\lambda_{i}}
$$

sont diffusés, après interaction avec l'échantillon, avec une énergie :

$$
E_{f}=\frac{\hbar^{2} k_{f}^{2}}{2 m_{n}}, k_{f}=\frac{2 \pi}{\lambda_{f}}
$$

dans une direction faisant un angle $\phi=2 \theta$ avec le faisceau incident. Lors de ce processus ils subissent un transfert de moment :

$$
\vec{Q}=\vec{k}_{i}-\vec{k}_{f}
$$

et un échange d'énergie :

$$
\hbar \omega=E_{i}-E_{f}
$$




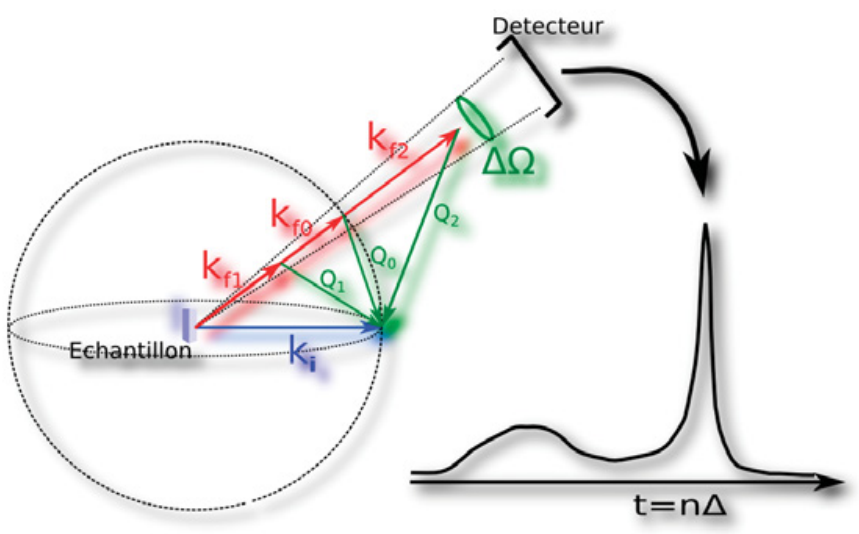

Figure 3. Schéma de principe d'une mesure inélastique par temps de vol: à une origine arbitraire des temps, un échantillon est brièvement éclairé par une bouffée de neutrons. On enregistre alors sur un détecteur équipé d'une horloge interne, la répartition du temps d'arrivée des neutrons diffusés: le spectre en temps de vol $\frac{d^{2} \sigma}{d \Omega d t}$ représenté ici en noir. L'échelle de temps est discrétisée en une succession de $\mathrm{n}$ canaux de temps de vol de largeur temporelle $\Delta \tau$ constante: $t=n . \Delta \tau$. L'interaction neutron-échantillon étant en général inélastique, les variables d'espace et d'énergie sont liées par la relation 3.25. Les trois configurations possibles sont représentées: $\left\|\vec{k}_{f_{1}}\right\|<\left\|\vec{k}_{i}\right\|:$ diffusion inélastique avec perte d'énergie du neutron (Stokes), $\left\|\vec{k}_{f_{0}}\right\|=\left\|\vec{k}_{i}\right\|:$ diffusion élastique, $\left\|\vec{k}_{f_{2}}\right\|>\left\|\vec{k}_{i}\right\|$ : diffusion inélastique avec gain d'énergie du neutron (anti-Stokes).

avec l'échantillon ${ }^{7}$. Les équations 3.3 et 3.4 constituent les lois de conservation du moment et de l'énergie au cours de la diffusion.

\subsection{Le temps de vol}

L'étape clef de la détermination de $S(\vec{Q}, \omega)$ par les méthodes de temps de vol consiste à mesurer l'énergie de chaque neutron diffusé, par l'évaluation de son temps de parcours, son temps de vol t, sur une distance connue. Dans le cadre de l'exemple présenté ici, il s'agit d'une simple mesure de $v_{f}$, la vitesse des neutrons diffusés, sur la distance échantillon-détecteur $L_{S D}$ :

$$
v_{f}=\frac{L_{S D}}{t}
$$

En d'autres termes, le spectre en temps de vol :

$$
\frac{d^{2} \sigma}{d \Omega d t}
$$

est la mesure, par unité d'angle solide $d \Omega$, de la fonction de distribution du temps d'arrivée, $t=n \Delta \tau$ ( $\Delta \tau$ est l'unité temporelle d'échantillonnage en temps), des neutrons sur le(s) détecteur(s) (Fig. 3). On montre [10] que la section efficace différentielle par élément d'énergie $d \omega$ et d'angle solide $d \Omega$ est directement reliée ${ }^{8}$ au facteur de structure dynamique $S(\vec{Q}, \omega)[10]$ :

$$
\frac{d^{2} \sigma}{d \Omega d \omega}=N \frac{k_{f}}{k_{i}} \frac{\sigma}{4 \pi} S(\vec{Q}, \omega)
$$

\footnotetext{
${ }^{7}$ Les signes de $\vec{Q}$ et $\hbar \omega$ sont fixés par convention.

8 On fait abstraction, ici, de l'existence de sections de diffusion cohérente et incohérente, pour ne considérer qu'une section de diffusion arbitraire $\sigma$.
} 


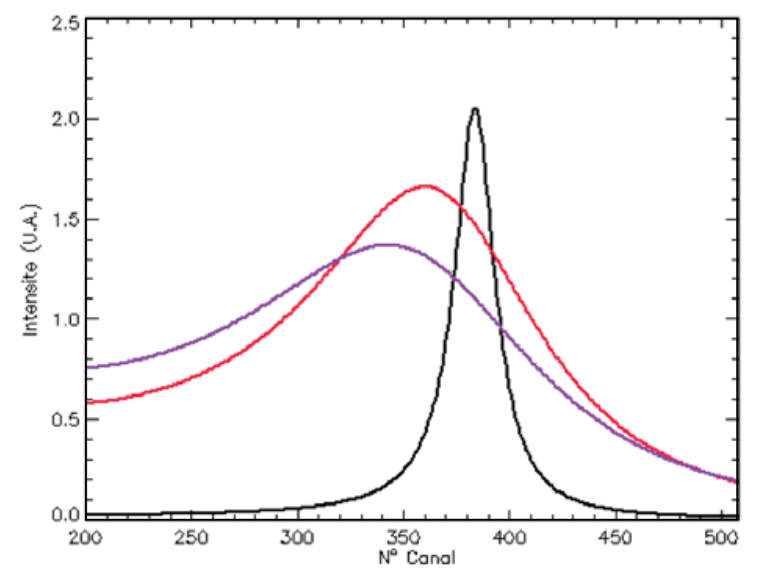

Figure 4. Exemple de spectre en temps de vol "brut" : dans la pratique on utilise un axe des abscises en numéro de canal, n (l'échelle en temps de vol t est obtenue par simple multiplication par la largeur d'un canal (channel width) $: t[\mu s]=n . \Delta \tau[\mu s])$. On a représenté un signal correspondant à un facteur de structure dynamique de forme lorentzienne $S(Q, \omega)=\frac{\Gamma}{\pi} /\left(\Gamma^{2}+\omega^{2}\right)$. Ce signal de type quasi-élastique est centré à transfert d'énergie nul, correspondant ici au canal élastique $n_{\text {élastique }}=385$. Trois valeurs de $\Gamma$ sont représentées : $0.05,0.3,0.4 \mathrm{meV}$ en noir, rouge et bleu respectivement (ici le spectre est simulé). En fonction de sa largeur à mi-hauteur, $\Gamma$, le spectre brut peut apparaître comme un signal inélastique, i.e. décalé par rapport au canal élastique (section 3.2). C'est une conséquence du terme en $t^{4}$ de l'équation 3.9.

En écrivant l'expression 3.6 sous la forme :

$$
\frac{d^{2} \sigma}{d \Omega d t}=\frac{d \omega}{d t} \frac{d^{2} \sigma}{d \Omega d \omega}
$$

en considérant que $E_{f}=\frac{1}{2} m_{n} v_{f}^{2}$, puis en combinant les équations 3.2 et 3.5 avec 3.7 , on obtient :

$$
\frac{d^{2} \sigma}{d \Omega d t}=\frac{N \sigma}{h \sqrt{E_{i}}}\left(\frac{m_{n}}{2}\right)^{\frac{3}{2}} \frac{L_{S D}^{3}}{t^{4}} S(\vec{Q}, \omega)
$$

Le spectre de temps de vol est donc directement lié au facteur de structure dynamique, mais sur des données brutes ce dernier apparaît "déformé" par un terme en temps de vol à la puissance 4 ème . Ceci peut avoir d'importantes conséquences sur l'allure des spectres bruts (Fig. 4). Le poids de ce terme en $1 / t^{4}$ devenant significatif quand t est grand, la soustraction très précise du bruit de fond électronique est essentielle. Ce point est discuté dans la section 3.4.

\subsection{Mise en oeuvre}

Ce sont les spectromètres de type "temps de vol-temps de vol" (voir aussi la section 5.1) qui permettent d'illustrer le plus simplement la mise en oeuvre d'une expérience de diffusion inélastique de neutrons par temps de vol. Les deux étapes essentielles de la mesure, la monochromatisation du faisceau incident puis l'analyse en énergie du faisceau diffusé font appel au même principe : la discrimination en énergie des neutrons selon le temps mis pour parcourir une distance connue, généralement de l'ordre de quelques mètres.

Monochromatisation : Un premier chopper muni d'une seule fente, tournant à la vitesse $\Omega_{c}$, «hache» le faisceau blanc ${ }^{9}$ incident en pulses temporels courts avec une fréquence $\nu[H z]=\Omega_{c}[\operatorname{tr} / \min ] / 60$

9 Par analogie avec la lumière, on désigne par faisceau blanc un faisceau présentant un spectre étendu en longueurs d'ondes et donc une distribution d'énergies. A l'opposé un faisceau monochromatique n'est composé que d'une seule longueur d'onde. 


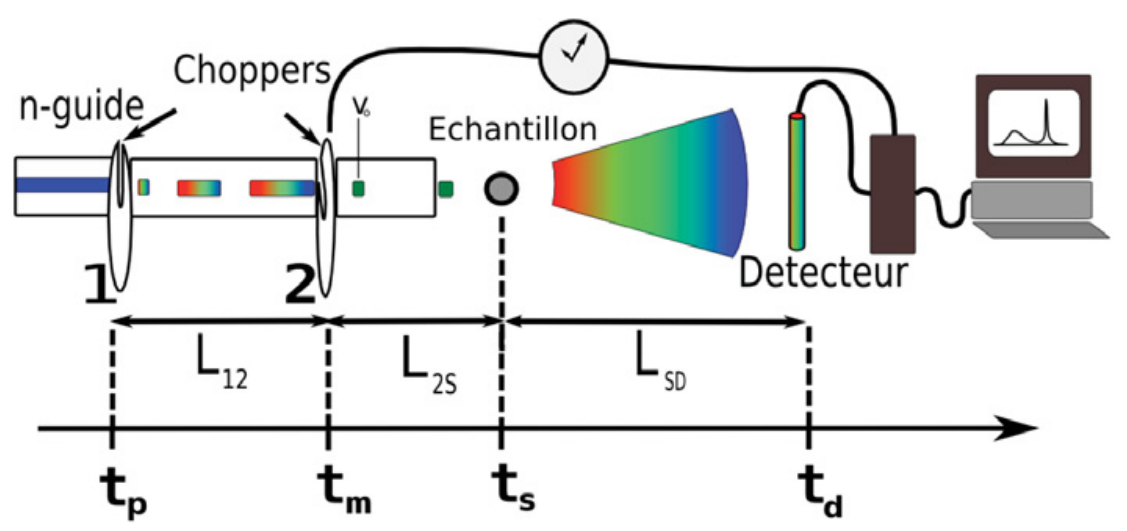

Figure 5. Schéma de principe d'un spectromètre à temps de vol illustrant les deux étapes cruciales de la mesure : la monochromatisation du faisceau blanc incident, puis l'analyse en temps de vol au niveau du détecteur (détails section 3.2.) Par souci de simplicité un seul détecteur est représenté. Sur un spectromètre réel, la mesure en temps de vol se fait simultanément sur plusieurs centaines de détecteurs (plus de 400 par exemple sur Mibémol, LLB, voir environ $10^{5}$ cellules indépendantes sur IN5, ILL avec les détecteurs à localisation).

(Fig. 5). A une distance $L_{12}$, un second chopper, identique au premier, mais déphasé d'un angle $\Phi$, reproduit cette opération de hachage du faisceau avec un décalage temporel $\delta[s]=\Phi / \nu[H z]$ modulo $1 / \nu$. Le choix de la valeur de $\Phi$ permet de sélectionner la longueur d'onde désirée. Seuls les neutrons de longueur d'onde :

$$
\lambda_{i}=\frac{h}{m_{n}} \frac{1}{L_{S D}} \frac{\Phi}{2 \pi \nu_{c}}
$$

qui parcourent la distance $L_{S D}$ pendant le temps $\delta$ sont transmis par le second chopper, les autres sont absorbés.

Analyse : L'instant de passage du faisceau par le deuxième chopper définit une référence des temps de la mesure (Fig. 5) à laquelle on déclenche l'horloge interne de la détection. Les neutrons diffusés par l'échantillon sont enregistrés en fonction de leur temps d'arrivée sur le détecteur. C'est la probabilité d'un échange d'énergie entre le neutron et l'échantillon, décrite par le facteur de structure dynamique $S(Q, \omega)$, qui induit cet étalement temporel des neutrons sur la distance échantillon-détecteur $L_{S D}$. Les neutrons ayant gagné de l'énergie arrivent les premiers au niveau du détecteur, suivis des neutrons diffusés élastiquement qui arrivent dans le canal (de temps de vol) "élastique" (Fig. 4). Enfin les neutrons ayant cédé de l'énergie à l'échantillon arrivent les derniers.

\subsection{Notion de résolution en énergie}

Le spectromètre élémentaire décrit au paragraphe précédent permet également d'illustrer une notion essentielle en spectroscopie inélastique par temps de vol : la notion de résolution en énergie. Les fentes des choppers n'étant pas infiniment fines, et les vitesses de rotation n'étant pas infinies, la longueur d'onde et donc l'énergie des neutrons sélectionnés par le système de choppers ne sont définies qu'à quelques pourcents. Si on considère une interaction neutron-échantillon purement élastique, l'incertidude sur la vitesse des neutrons incidents induit une incertitude sur leur temps d'arrivée. Le pic élastique n'est pas infiniment fin, mais présente une largeur intrinsèque : la résolution en énergie de l'instrument également appelée fonction d'appareil (un cas réel est représenté sur la figure 6). C'est essentiellement ce paramètre qui contrôle le temps de corrélation le plus long mesurable dans une configuration instrumentale donnée. 


\subsubsection{Un calcul analytique simple}

Au prix de quelques approximations ${ }^{10}$, un raisonnement assez simple permet de calculer analytiquement la résolution. Les vitesses des neutrons avant $\left(v_{i}\right)$ et après $\left(v_{f}\right)$ l'échantillon s'expriment en fonction des paramètres définis sur la figure 5 :

$$
\begin{aligned}
v_{i} & =\frac{L_{12}}{\left(t_{m}-t_{p}\right)}=\frac{L_{12}+L_{2 S}}{\left(t_{s}-t_{p}\right)} \\
v_{f} & =\frac{L_{S D}}{\left(t_{d}-t_{s}\right)}
\end{aligned}
$$

d'autre part :

$$
t_{s}=t_{p}+\frac{L_{12}+L_{2 S}}{v_{i}}=\left(1+\frac{L_{2 S}}{L_{12}}\right) t_{m}-\left(\frac{L_{2 S}}{L_{12}}\right) t_{p}
$$

On exprime donc le transfert d'énergie en fonction des temps de parcours :

$$
\begin{aligned}
\hbar \omega & =\frac{1}{2} m_{n}\left(v_{i}^{2}-v_{f}^{2}\right) \\
& =\frac{1}{2} m_{n}\left[\frac{L_{12}^{2}}{\left(t_{m}-t_{p}\right)^{2}}-\frac{L_{S D}^{2}}{\left(t_{d}-\left(1+\frac{L_{2 S}}{L_{12}}\right) t_{m}+\left(\frac{L_{2 S}}{L_{12}}\right) t_{p}\right)^{2}}\right]
\end{aligned}
$$

L'incertitude sur le transfert d'énergie résulte alors des incertitudes sur les mesures des temps $t_{i, i=p, m, s, d}$. En supposant que ces incertitudes sont assimilables à des variables normales non corrélées et qu'elles s'additionnent donc en quadrature :

$$
\delta \hbar \omega=\left[\sum_{i=p, m, s, d}\left(\frac{\partial \hbar \omega}{\partial t_{i}}\right)^{2} \delta t_{i}^{2}\right]^{\frac{1}{2}}
$$

Calculant les dérivées partielles dans (3.14) et définissant $C=m_{n} / h=252.8 \mu \mathrm{s} . \mathrm{m}^{-1} \AA^{-1}$ :

$$
\begin{aligned}
t_{m}-t_{p} & =C L_{12} \lambda_{i} \\
t_{d}-t_{m} & =C\left(L_{2 S} \lambda_{i}+L_{S D} \lambda_{f}\right) \\
t_{d}-t_{s} & =C L_{S D} \lambda_{f}
\end{aligned}
$$

on obtient :

$$
\delta \hbar \omega=\frac{m}{C^{3} L_{12} L_{S D} \lambda_{f}^{3}}\left\{\begin{array}{l}
{\left[L_{2 S}+L_{S D}\left(\frac{\lambda_{f}}{\lambda_{i}}\right)^{3}\right]^{2} \delta t_{p}^{2}+} \\
{\left[\begin{array}{l}
\left.L_{12}+L_{2 S}+L_{S D}\left(\frac{\lambda_{f}}{\lambda_{i}}\right)^{3}\right]^{2} \delta t_{m}^{2}+ \\
L_{12}^{2} \delta t_{d}^{2}
\end{array}\right\}}
\end{array}\right.
$$

Les incertitudes $\delta t_{p}$ et $\delta t_{m}$ s'identifient aux temps d'ouverture des premier et dernier choppers et $\delta t_{d}$ à l'incertitude sur le temps de vol échantillon-détecteur. En gardant à l'esprit que $\lambda_{f} / \lambda_{i}=(1-$ $\left.\hbar \omega / E_{i}\right)^{-1 / 2}$, il apparaît donc que $\delta \hbar \omega$ dépend fortement de $\hbar \omega$.

\footnotetext{
10 On assimile les sources d'incertitudes à des variables normales non corrélées dont les écarts-types s'additionnent en quadrature (cf. Eq. (3.15)).
} 
En dérivant l'énergie cinétique du neutron diffusé et avec $\delta t^{2}=\sum_{i} \delta t_{i}^{2}$ on obtient une relation entre la résolution en énergie, en longueur d'onde et en temps. La relation 3.17 est rigoureusement identique à celle souvent citée[11-13] :

$$
\frac{\delta \hbar \omega}{E_{f}}=2 \frac{\delta \lambda}{\lambda_{f}}=2 \frac{\delta t}{t}
$$

Cette relation montre que physiquement, la résolution est la largeur du pic élastique au niveau du détecteur. On verra que cette constatation est essentielle pour comprendre le principe de la focalisation en temps.

A un transfert d'énergie $\hbar \omega_{\circ}$, la fonction d'appareil du spectromètre peut être approximée par une gaussienne :

$$
\Re(\omega)=\frac{1}{\sqrt{2 \pi} \delta \hbar \omega} e^{-\frac{1}{2}\left[\left(\hbar \omega-\hbar \omega_{0}\right) / \sigma\right]^{2}}
$$

Dans la pratique, plutôt que par son écart type $\sigma$, on définit cette gaussienne par sa largeur totale à mi-hauteur (FWHM pour Full Width at Half Maximum) :

$$
\delta \hbar \omega=2 \sqrt{2 * \ln (2)} . \sigma \approx 2.35 \sigma
$$

Expérimentalement, on mesure un spectre, conséquence de la convolution de la fonction de diffusion $\mathrm{S}(\vec{Q}, \omega)$ avec cette fonction de résolution :

$$
\begin{aligned}
\left(\frac{d^{2} \sigma}{d \Omega d \omega}\right)_{E x p} & =N \frac{k_{f}}{k_{i}} \frac{\sigma}{4 \pi} \int_{-\infty}^{+\infty} S\left(\vec{Q}, \omega^{\prime}\right) \Re\left(\omega-\omega^{\prime}\right) d \omega^{\prime} \\
& =N \frac{k_{f}}{k_{i}} \frac{\sigma}{4 \pi} S(\vec{Q}, \omega) \otimes \Re(\omega)
\end{aligned}
$$

Il est fréquent que l'on considère que dans la zone quasi-élastique du spectre i.e. sur une gamme de l'ordre de quelques meV de part et d'autre du pic élastique, la résolution ne dépend que faiblement du transfert d'énergie i.e. $\sigma$ n'est pas fonction de $\hbar \omega$.

\subsubsection{Influence sur la gamme temporelle accessible}

L'analyse de l'expression (3.17) conduit à faire deux observations importantes :

- la résolution $\mathfrak{R}(\omega)$ dépend fortement du transfert d'énergie, mais par convention, le terme résolution en énergie d'un spectromètre désigne la largeur totale à mi-hauteur du pic élastique i.e. à $\hbar \omega=0 . \Re(\hbar \omega)$ est en général déterminée expérimentalement par la mesure d'un diffuseur purement élastique (cf. section 4.1). Un exemple de résolution instrumentale est donnée sur la figure 6. La forme exacte de cette dépendance en $\hbar \omega$ est très liée à la technique utilisée, mais dans tous les cas, à grands transferts d'énergie la résolution est dominée par l'incertitude sur le temps de vol.

- Au premier ordre, la résolution en énergie d'un spectromètre varie en $\lambda_{i}^{-3}$. Le meilleur moyen d'augmenter la résolution, i.e. diminuer $\delta \hbar \omega$, est d'augmenter la longueur d'onde. Il y a bien sûr un coût à payer en terme de temps de comptage, puisque le flux délivré par le guide chute en puissance de $\lambda_{i}, \lambda_{i}^{-n},(n=4,5$ suivant le guide $)$ au delà d'un maximum dont la position dépend de la température de la source froide et du type de guide (de $\approx 4$ à $6 \AA$ ).

Si on fait abstraction de toute influence de la statistique de la mesure, c'est la résolution en énergie qui contrôle le temps de corrélation le plus long mesurable dans une configuration donnée. Cet effet 
Tableau 1. Distances caractéristiques de quelques temps de vol à choppers. $L_{12}$ est la distance entre le premier et le dernier chopper (ou entre le milieu de la première et dernière paire), $L_{2 S}$ est la distance dernier chopper (milieu de la dernière paire) - échantillon et $L_{S D}$ la distance de vol échantillon - détecteur.

\begin{tabular}{lccc}
\hline Instrument & $L_{12}$ & $L_{2 S}$ & $L_{S D}$ \\
\hline IN5 & 8.0 & 1.2 & 4.0 \\
MIBEMOL & 9.0 & 1.4 & 3.6 \\
DCS & 9.0 & 1.5 & 4.01 \\
TOFTOF & 10.0 & 1.4 & 4.0 \\
NEAT & 12.0 & 1.3 & 2.5 \\
\hline
\end{tabular}

apparaît clairement quand on se place dans l'espace des temps, par transformée de Fourier inverse de l'expression 3.23. La fonction intermédiaire de diffusion est :

$$
\begin{aligned}
I(\vec{Q}, t)_{E x p} & =\frac{1}{2 \pi} \int_{-\infty}^{+\infty}(S(\vec{Q}, \omega) \otimes \Re(\omega)) e^{i \omega t} d \omega \\
& =I(\vec{Q}, t) . \Re(t)
\end{aligned}
$$

Le produit de convolution de l'Eq. (3.23) apparaît désormais comme un produit et la gaussienne de résolution $\Re(\omega)$, comme une gaussienne en temps $\Re(t)$ dont l'écart type $\sigma_{t}=\hbar / \delta \hbar \omega$ est le facteur limitant la mesure des temps de corrélations longs. En d'autres termes, plus le pic élastique est étroit, i.e. plus $\delta \hbar \omega$ est petit, plus $\sigma_{t}$ est grand et plus il est possible d'explorer des temps longs. Dans la pratique, on estime que le signal $\mathrm{I}(\mathrm{Q}, \mathrm{t})$ est détectable jusqu'à $2 \sigma_{t}$ (c'est-à-dire que l'intensité est encore significative à $2 \sigma_{t}$ ). Le temps le plus long accessible est donc de l'ordre de $t_{\operatorname{Max}}[p s]=$ $2 * 0.658 / \delta \hbar \omega[\mathrm{meV}]$. A titre d'exemple, une résolution élastique de $24 \mu \mathrm{eV}$ peut permettre de mesurer des temps de l'ordre de $2 * 0.658 * 2 \sqrt{2 \ln (2)} / 0.024=130$ ps (on a utilisé l'équation 3.20).

\subsection{Ordres de grandeurs}

Ce paragraphe précise les ordres de grandeurs de certains paramètres instrumentaux clefs.

- Pourquoi des spectromètres si grands? : Les longueurs d'onde incidentes utilisées sur les machines à temps de vol sur neutrons froids couvrent une gamme de $\approx 2$ à $\approx 20 \AA$. Une résolution relative $\delta \hbar \omega / E$ de quelques pourcents (par exemple, sur Mibémol : $96 \mu \mathrm{eV}$ et $24 \mu \mathrm{eV}$ pour $\lambda_{i}=6$ Å et $9 \AA$ A , c'est à dire $3.9 \%$ et $2.8 \%$ ) est adaptée à l'étude de la plupart des processus physiques en matière condensée. L'application numérique de l'équation (3.17) montre que l'obtention de telles incertitudes relatives impose des distances caractéristiques de l'ordre de plusieurs mètres (voir tableau 1 et section 5.1.3).

- Des pulses de l'ordre de quelques dizaines de $\mu s$ : La vitesse de rotation des choppers étant de l'ordre de 1000 à $20000 \mathrm{tr} / \mathrm{min}$, la fréquence de répétition de la mesure est de l'ordre de 20 à $300 \mathrm{~Hz}$. Des raffinements existent, mais à l'ordre 0 , les fenêtres aménagées dans les disques des choppers correspondent en général à la taille du guide i.e. environ Largeur*Hauteur $=2.5 \times 5 \mathrm{~cm}^{2}$. Le temps d'ouverture de chaque fente est donc de 50 à $100 \mu$ s et au niveau de l'échantillon, la durée du pulse est de quelques dizaines de microsecondes, ce qui correspond à quelques canaux de temps de vol de largeur typique $\Delta \tau=10-20 \mu$ s. Cette combinaison d'échelles de temps permet une bonne définition du pic élastique. Un neutron $\lambda_{i}=\lambda_{f}=6 \AA$ parcourant la distance échantillon détecteur en $6 \mathrm{~ms}$, la période de mesure de 3 à $6 \mathrm{~ms}$ imposée par les choppers n'est donc pas assez longue pour détecter des neutrons ayant cédé de l'énergie à l'échantillon. Ceci fait apparaitre que, pour être 
parfaitement opérationnel, le spectromètre simpliste décrit dans ce chapitre, nécessite quelques perfectionnements : l'ajout de choppers pour permettre d'écarter les pulses ou au contraire l'ajout de fentes... Ces aspects sont détaillés dans la section 5.1.

- Une échelle de mesure dans la $\mu s$ pour accéder à des ps : Un point essentiel mérite d'être souligné : c'est par la mesure de temps de l'ordre de la dizaine de microsecondes que les spectromètres à temps de vol permettent d'accéder à des temps de corrélation aussi courts que quelques fractions de picosecondes.

- Un spectromètre sur mesure : adapter l'échelle en temps de vol à sa physique. Afin d'utiliser au mieux le flux délivré par la source, l'échelle de temps de vol est ajustée de façon à (i) détecter la totalité des neutrons diffusés, quelque soit leur échange d'énergie et (ii) éviter tout temps mort entre deux spectres en temps de vol consécutifs.

Une convention a été parfois adoptée [16] consiste à considérer qu'après interaction avec l'échantillon, les longueurs d'ondes extrêmes traversant la base de temps de vol sont $\lambda_{\text {Max }}=1.5$ $\lambda_{i}$ et $\lambda_{\text {Min }}=0.1$.

- le terme $1 / t^{4}$ de l'équation 3.9 a pour conséquence que quelle que soit la forme de $S(Q, \omega)$, il n'y a statistiquement qu'une fraction négligeable de neutrons de longueur d'onde supérieure à $\lambda_{\text {Max }}$.

- $\lambda_{\text {Min }}$ correspond à un échange d'énergie de plus de $8000 \mathrm{meV}(40 \mathrm{~km} / \mathrm{s})$ i.e. bien supérieur à ceux mis en jeu dans les excitations de la matière condensée. Les événements détectés dans les premiers canaux où se situeraient les neutrons ayant une telle énergie correspondent donc à du bruit de fond (partie non structurée à temps court du spectre schématisé sur la figure 3). Ce bruit de fond est ajusté par une droite pour être soustrait lors du traitement de données. C'est une façon simple de l'estimer sachant qu'il est essentiel de le soustraire en raison du terme en $t^{4}$ qui apparaît lors de la transformation permettant de passer des données brutes à $S(Q, \omega)$ (cf. Eq. (3.9)).

Le facteur de structure dynamique mesuré est affecté par la température $\mathrm{T}$ de l'échantillon selon le facteur de population de Bose, $n_{B}(\omega, T)=\left(\exp \left(-\frac{\hbar \omega}{k_{B} T}\right)-1\right)^{-1}$. C'est ce produit $S(Q, \omega) \cdot n_{B}(\omega, T)$, qui contrôle donc $\lambda_{\text {Min }}$. La règle ci-dessus est donc très générale et n'est pas forcément pertinente pour optimiser l'échelle de temps de vol à basse température.

- De quelques centaines à quelques milliers de neutrons détectés par seconde. Sur le réacteur Orphée au LLB le flux de neutrons de fission au niveau du coeur est de l'ordre est de l'ordre de $10^{14} \mathrm{n} / \mathrm{cm}^{2} / \mathrm{s}$, après modération par la source froide et passage dans le guide on tombe à $10^{9}$ $\mathrm{n} / \mathrm{cm}^{2} / \mathrm{s}$ à l'entrée du spectromètre. La nécessité de pulser le faisceau conduit ensuite à une chute brutale d'intensité au niveau de l'échantillon : environ $\Phi_{E c h}=10^{4} \mathrm{n} / \mathrm{cm}^{2} / \mathrm{s}$. Pour un réacteur comme HFR à l'ILL Grenoble ou FRM-II à Garching près de Munich, les chiffres ci-dessus sont typiquement un ordre de grandeur plus élevés.

Pour éviter des problèmes de diffusion multiple, on choisit l'épaisseur de l'échantillon $\left(\right.$ Surface $_{\text {Echantillon }}=$ Largeur $\times$ Hauteur $=2.5 \times 5 \mathrm{~cm}^{2}$ ), de façon à obtenir une transmission de l'ordre de $\mathcal{T}=90 \%$. L'angle solide de détection pour le spectromètre MIBEMOL au LLB (surface de detection $S_{D e t}=5 \mathrm{~m}^{2}$ ) est de le l'ordre de $0.4 \mathrm{st}$ à $L_{S D}=3.6 \mathrm{~m}$ de l'échantillon. La fréquence de détection est donc de l'ordre de $\left.(1-\mathcal{T}) * S_{D e t} / 4 \pi / L_{S D}^{2} * \Phi_{E c h}\right)=25 \mathrm{n} / \mathrm{cm}^{2} / \mathrm{s}$ soit $\approx 310 \mathrm{n} / \mathrm{s}$. Le niveau de bruit de fond total est de l'ordre de quelques coups par seconde sur l'ensemble du détecteur. Sur les spectromètres optimisés de dernière génération de type IN5 (ILL) où en particulier la surface de détection est de l'ordre de $30 \mathrm{~m}^{2}$ et les flux incidents bien plus importants, ces chiffres sont près de deux ordres de grandeur supérieurs (sauf pour le bruit de fond qui est typiquement de moins de 1 neutron par seconde et par mètre carré de détecteur). 

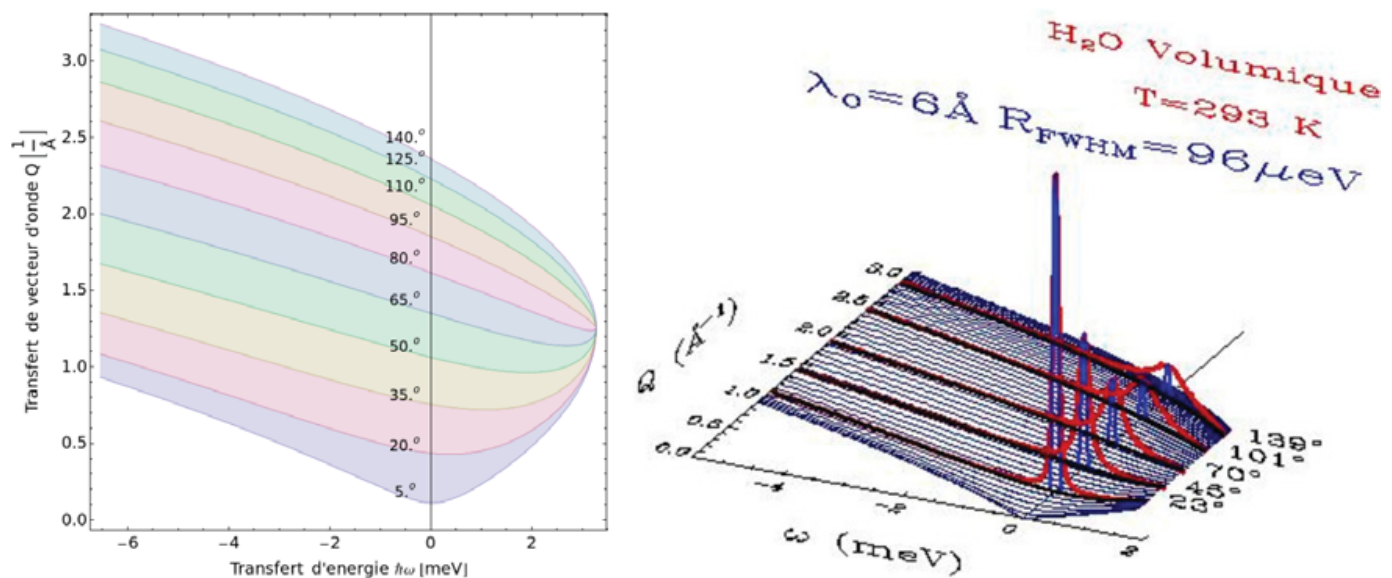

Figure 6. (a) Représentation des lignes à angle constant dans l'espace $(Q, \hbar \omega)$ d'après l'équation 3.26 avec $\lambda_{i}=5.2 \AA$. Selon la convention de signe choisie, les valeurs positives de $\hbar \omega=E_{i}-E_{f}$ correspondent à une perte d'énergie du neutron. La perte d'énergie maximale du neutron est bornée par son énergie initiale, ici $E_{i}=3.0 \mathrm{meV}$. b) Spectre de l'eau volumique mesuré sur Mibémol (LLB). La longueur d'onde incidente est $\lambda_{i}=6$. Les valeurs positives de $\hbar \omega=E_{i}-E_{f}$ correspondent à une perte d'énergie des neutrons. Le transfert maximal d'énergie est défini par l'énergie incidente, ici $\hbar \omega_{\text {Max }}=E_{i}=1 / 2 . h^{2} / m_{n} \cdot \lambda_{i}^{-2}=2.25 \mathrm{meV}$. Pour la lisibilité de la figure, sur les 84 spectres mesurés simultanément entre 20 et $140^{\circ}$, seules les données recueillies à quatre angles de diffusion $\phi=23,45,70$ et $139^{\circ}$ sont représentées (courbes rouges). A chaque fois, la fonction de résolution de l'instrument $(\mathrm{R}=96 \mu \mathrm{eV})$ est représentée en bleu à $\hbar \omega=0$ et la courbe paramétrique $Q(\phi, \omega)(\mathrm{Eq}$. (3.26)) est repérée en gras dans le plan $(Q, \omega)$. Les traits plus fins représentés dans ce plan correspondent aux courbes $Q(\phi=0,4,8 \ldots 180, \omega)$. Il apparaît clairement qu'une mesure en temps de vol ne se fait pas à module de vecteur de diffusion $\mathrm{Q}=|\vec{Q}|$ constant.

\subsection{Transfert de moment}

Le module du vecteur de diffusion $Q=|\vec{Q}|$, l'angle de diffusion $\phi=2 \theta$, et le transfert d'énergie $\hbar \omega$ sont liés par la relation :

$$
\begin{aligned}
Q^{2} & =k_{i}^{2}+k_{f}^{2}-2\left|\vec{k}_{i}\right|\left|\vec{k}_{f}\right| \cos (\phi) \\
& =2\left[\frac{2 \pi}{\lambda_{i}}\right]^{2}\left(1-\frac{\hbar \omega}{2 E_{i}}-\sqrt{1-\frac{\hbar \omega}{E_{i}}} \cos (\phi)\right)
\end{aligned}
$$

Deux canaux de temps de vol successifs d'un détecteur orienté à $\phi=2 \theta$ sondent donc la matière à des échelles $Q(\phi, \omega)$ légèrement différentes. Associer une valeur de $\mathrm{Q}$ précise à un détecteur sous-entend que l'on considère la norme du vecteur de diffusion à transfert d'énergie nul $Q_{0}=Q(\phi, \omega=0)=$ $\frac{4 \pi}{\lambda_{i}} \sin (\phi / 2)$. Le passage de données de temps de vol à une représentation à $Q(\phi, \omega)$ constant nécessite l'interpolation de la nappe $S(\phi, \omega)$ (Fig. 6). Cette transformation n'est réalisable que sur des données présentant une excellente statistique et occasionne dans tous les cas une perte d'information. Mis à part pour faciliter la visualisation des données et dans les cas où une modélisation analytique est difficile (pour la diffusion magnétique par exemple), cette opération n'est effectuée que si elle est vraiment nécessaire.

Le signal enregistré à un angle donné est en général la somme de l'intensité détectée sur plusieurs détecteurs distincts, des tubes de l'ordre du centimètre de diamètre et de 20 à $30 \mathrm{~cm}$ de hauteur, disposés de part et d'autre du plan de diffusion et branchés en série. Pour limiter tout effet d'interpolation, on prend soin d'orienter les détecteurs selon des cônes de Debye-Scherrer. Cet arrangement est très adapté 

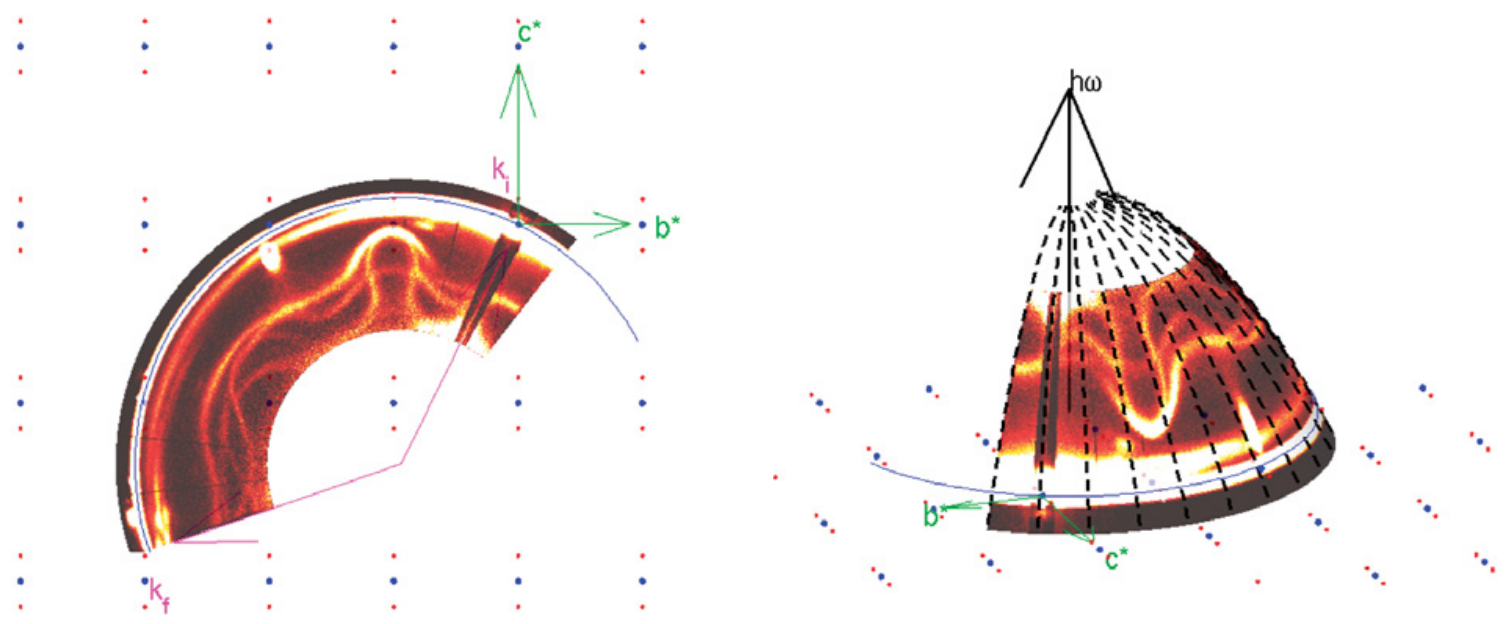

Figure 7. Spectre de diffusion dans le plan équatorial d'un monocristal de $\mathrm{Ba}_{3} \mathrm{NbFe}_{3} \mathrm{Si}_{2} \mathrm{O}_{14}$ (BNFS) projeté dans son espace réciproque[14]. Le spectre montre les dispersions des magnons côté Stokes à basse température $(2 \mathrm{~K})$ autour des satellites magnétiques (points rouges), la contribution nucléaire (pic de Bragg nucléaires en bleu) étant négligeable dans ce cas. Pour chaque orientation de l'échantillon, donc chaque spectre enregistré, la nappe $(\vec{Q}, \omega)$ de l'instrument fait une coupe parabolique dans les branches de dispersion. Ici seule la diffusion Stokes est retenue (la diffusion élastique est matérialisée par le cercle bleu). L'association de plusieurs orientations échantillon permet de reconstituer les dispersions dans toutes les directions de l'espace réciproque, y compris les directions de haute symétrie (voir figure 7).

à des échantillons liquides, amorphes et cristallins de type poudre mais très peu aux échantillons monocristallins puisqu'il ne permet pas le positionnement précis de pics de Bragg. Depuis l'avènement récent de grandes surfaces de détection de type PSD (Position Sensitive Detector), cette limitation technique est désormais levée sur les appareils de dernière génération (MAPS à ISIS et IN5 à l'ILL).

\subsection{Diffusion inélastique par temps de vol et trois axes}

Dans la gamme d'énergie de l'ordre du meV (figure 2), principalement deux techniques permettent de mettre en œuvre des expériences de diffusion inélastique de neutrons : les spectromètres à temps de vol et les spectromètres trois axes. Comme on l'a vu en introduction, historiquement, ils ont été développés conjointement sur des réacteurs, donc sur des sources continues.

La technique trois axes permet un comptage continu de l'intensité diffusée, et ces spectromètres sont donc par nature très adaptés aux réacteurs. A l'inverse, la technique par temps de vol qui, pour procéder à la mesure de vitesse, nécessite la définition d'un intervalle de temps, conduit à artificiellement pulser la source. Sur une source continue, cette contrainte se traduit par une diminution d'environ deux ordres de grandeur du flux intégré au niveau de l'échantillon (le nombre total de neutrons vus par un échantillon est de -pour fixer les idées- $10^{7} \mathrm{n} / \mathrm{cm}^{2} / \mathrm{s}$ sur un 3 axes et $10^{5} \mathrm{n} / \mathrm{cm}^{2} / \mathrm{s}$ sur un temps de vol). Sur ces appareils, cette perte de flux est compensée par l'utilisation d'une grande surface de détection. En conséquence, en temps de vol, on utilise la résolution spatiale à transfert de moment $|\vec{Q}|$ constant (Eq. (3.3)) en regroupant les détecteurs en cônes de Debye-Scherrer afin de gagner en statistique. Cette contrainte technique est en partie responsable de la scission des thématiques scientifiques abordées sur trois axes et temps de vol : l'étude de phénomènes inélastiques mettant en jeux des systèmes monocristallins, qui nécessite un positionnement fin dans l'espace réciproque et donc aussi une bonne résolution en $\vec{Q}$, est très active sur les trois axes. Les phénomènes physiques caractérisées par des dépendances en $\mathrm{Q}$ faibles ou variant lentement comme dans les systèmes liquides ou amorphes sont plutôt abordés sur des temps de vol. 

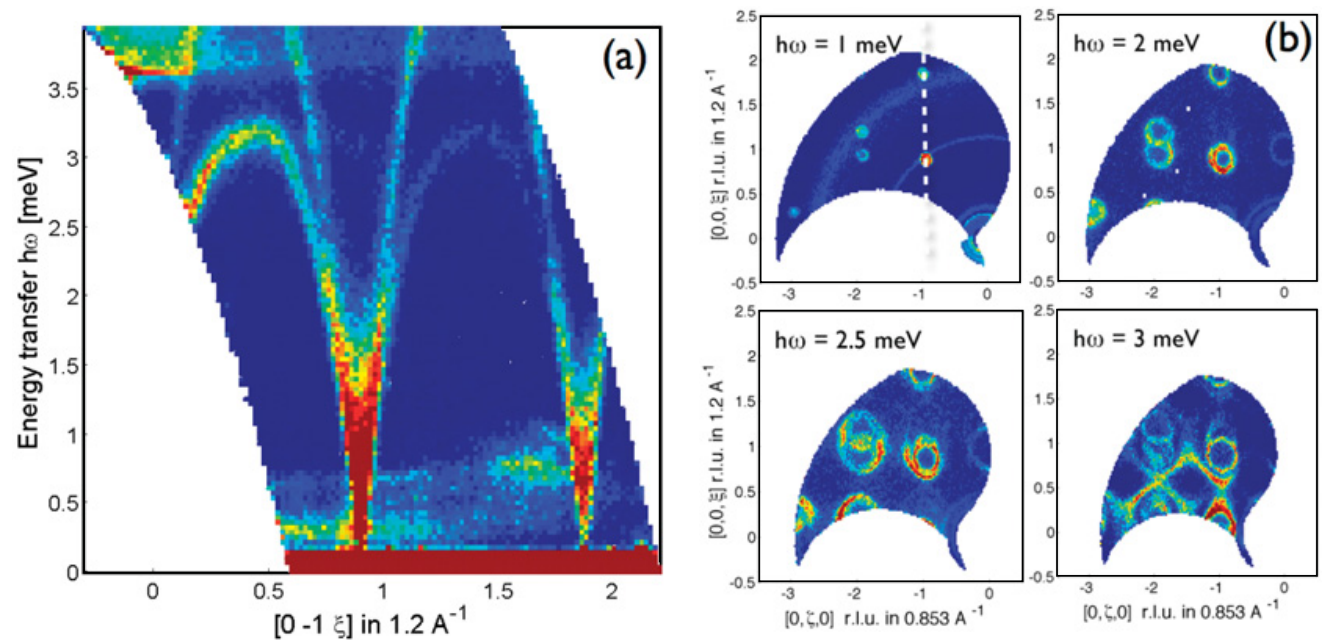

Figure 8. (a) Courbes de dispersion des magnons de BNFS à $\mathrm{T}=2 \mathrm{~K}$ selon une coupe le long d'une direction de haute symétrie $(0, \overline{1}, \zeta)$ de l'espace réciproque. (b) Coupes iso-énergies dans le plan $\left(\mathrm{b}^{*}, \mathrm{c}^{*}\right)$ intégré sur $0.1 \mathrm{meV}$. Les courbes de dispersion (a) correspondent à la ligne verticale en pointillés de la figure (b). Les figures sont des coupes à 2 dimensions de données à 4 dimensions obtenues par l'assemblage de 80 spectres de temps de vol comme celui présenté sur la figure (7).

Cette fracture thématique d'origine purement technique, est en partie réduite avec l'avènement de grandes surfaces de détection formées de tubes à localisation ou PSD (Position Sensitive Detector).

Les détecteurs à localisation sont des tubes cylindriques similaires à ceux des détecteurs unitaires mais plus longs (actuellement des détecteurs de $5 \mathrm{~m}$ ont été testés) ${ }^{11}$. Remplis d'hélium 3 et d'un gaz de "freinage" (la plupart du temps du $\mathrm{CF}_{4}$ ) comme des détecteurs unitaires ordinaires, l'astuce supplémentaire revient à mesurer la chute de tension aux deux bouts du détecteur entre anode et cathode et, connaissant la résistivité du fil central d'anode en déduire la position de la détection le long du tube par la méthode du pont de résistance (ou de capacitance) $: x=\frac{V_{1}}{V_{1}+V_{2}}$. La précision de localisation est de l'ordre du centimètre pour des détecteurs de 1 pouce de diamètre sur $3 \mathrm{~m}$ de long.

Les PSDs permettent alors d'obtenir des résolutions spatiales du même ordre que sur les 3 axes et permettent d'explorer de grandes portions de l'espace réciproque d'un monocristal en un seul spectre (figure 7). L'exploration de directions particulières de hautes symétries du cristal nécessite toutefois l'assemblage de plusieurs spectres correspondant à des orientations de cristal différentes ou à des longueurs d'onde incidentes différentes. On extrait alors des coupes à 1,2 ou 3 dimensions des données à 4 dimensions $\left(Q_{x}, Q_{y}, Q_{z}, \hbar \omega\right)$ difficilement exploitables directement (figure 8).

Contrairement aux spectromètres à trois-axes, l'analyse des données dans des directions particulières ne peut se faire qu'après enregistrement d'un jeu complet de données; l'analyse dans des directions particulières ne pouvant se faire qu'après assemblage des spectres. Bien que puissante, la méthode est jugée parfoit moins interactive que sur un spectromètre trois axes.

\section{PRATIQUE D'UNE MESURE PAR TEMPS DE VOL}

Avant de pouvoir obtenir les données sous la forme du facteur de structure dynamique $S(\phi, \omega)$ ou $S(Q, \omega)$ les données brutes doivent être corrigées des biais expérimentaux, induits soit par l'instrument, soit par l'échantillon lui-même. La pertinence des corrections citées ci-dessous dépend du type

${ }^{11}$ MAPS (ISIS) : $1 \mathrm{~m}$ et $1.3 \mathrm{~m}$, IN5 (ILL) et MERLIN (ISIS) : $3 \mathrm{~m}$, LET (ISIS) $: 4 \mathrm{~m}, \ldots$ 
d'échantillon, de la précision recherchée et de l'objectif de l'expérience. Elles peuvent être essentielles si l'on cherche à mesurer une grandeur physique de façon absolue, ce qui est une situation plutôt rare : dans la pratique, on observe plutôt des variations relatives d'une propriété en fonction d'un paramètre. Plus qu'une règle absolue, l'application de ces corrections définit une "ligne de conduite" à suivre lors du traitement des données.

\subsection{Corrections instrumentales et normalisation}

Il s'agit des corrections intrinsèques à l'instrument :

$\rightarrow$ Correction du bruit de fond instrumental - La première opération consiste à soustraire le bruit de fond instrumental pour obtenir le signal de l'échantillon $S$ dans sa cellule. Ce qui impose de mesurer :

- Un échantillon $S$ dans son porte échantillon : $X_{S}=B_{1}+S+\mathcal{T} \cdot B_{2}$

- Un environnement échantillon vide $E: E=B_{1}+B_{2}$

- Un cadmium $C$ pour évaluer le bruit de fond d'ambiance (i.e. indépendant de la présence du faisceau diffusé par l'échantillon dans le spectromètre) : $C=B_{1}$. Alors que le terme $C$ dépend principalement du temps de comptage, toutes les autres contributions sont fonctions du moniteur.

$B_{1}$ et $B_{2}$ sont les bruits de fond générés par le faisceau avant et après l'échantillon. Lorsque le faisceau traverse l'échantillon, le bruit de fond généré après l'échantillon (plus son container) est pondéré par la transmission $\mathcal{T}$ de l'échantillon. On a alors d'après les équations ci-dessus :

$$
S=X_{S}-\mathcal{T} \cdot E-(1-\mathcal{T}) C
$$

$\rightarrow$ Normalisation des détecteurs - Les différents détecteurs pouvant avoir des "efficacités" variables (pression et composition du gaz, masquage partiel du détecteur,...) il est nécessaire de les normaliser grâce à une mesure de référence d'un diffuseur isotrope, en général l'intégrale du pic élastique purement incohérent du vanadium :

$$
I_{V}(\phi, \omega=0)=\epsilon\left(\phi, \lambda_{i}\right) S_{V}(\phi, \omega=0)
$$

où :

$$
S_{V}(\phi, \omega=0)=e^{-\left\langle u_{v}^{2}\right\rangle Q_{e}^{2}}
$$

Cette dernière quantité, le facteur de structure dynamique à transfert d'énergie nul du vanadium, est théoriquement d'intensité normalisée pourvu qu'elle soit corrigée du terme de Debye-Waller $\exp \left(-\left\langle u_{v}^{2}\right\rangle Q_{e}^{2}\right)$ qui rend compte de la température de mesure du vanadium. Le paramètre $\epsilon\left(\phi, \lambda_{i}\right)$ prend en compte la différence d'efficacité des détecteurs en fonction de l'angle de diffusion pour l'énergie incidente choisie (puisque l'on prend l'intégrale du pic élastique).

$\rightarrow$ Efficacité des détecteurs - La gamme dynamique des neutrons diffusés pouvant être très étendue, la dépendance en énergie de l'efficacité des détecteurs est prise en compte. C'est un paramètre qui dépend (i) de la pression de gaz de détection, en général de l'hélium $3\left({ }^{3} \mathrm{He}\right)$, mais aussi (ii) de l'absorption et (iii) de la diffusion - et, aux longueurs d'onde courtes ( $E_{f}$ grand), de la diffraction - par les parois des détecteurs. Elle dépend dans une moindre mesure de la géométrie des détecteurs. Le calcul exact n'étant pas forcément trivial, on approxime l'ensemble par une fonction empirique [18] :

$$
\epsilon\left(\frac{\lambda_{f}}{\lambda_{i}}\right)=A e^{-a \lambda_{f}}\left(1-e^{-b \lambda_{f}}\right)
$$

où les coefficients $A, a, b$ sont ajustés pour chaque type de détecteur, par exemple en comparant les intensités à différentes longueurs d'ondes avec les intensités obtenues à l'aide d'un moniteur calibré (figure 9). 


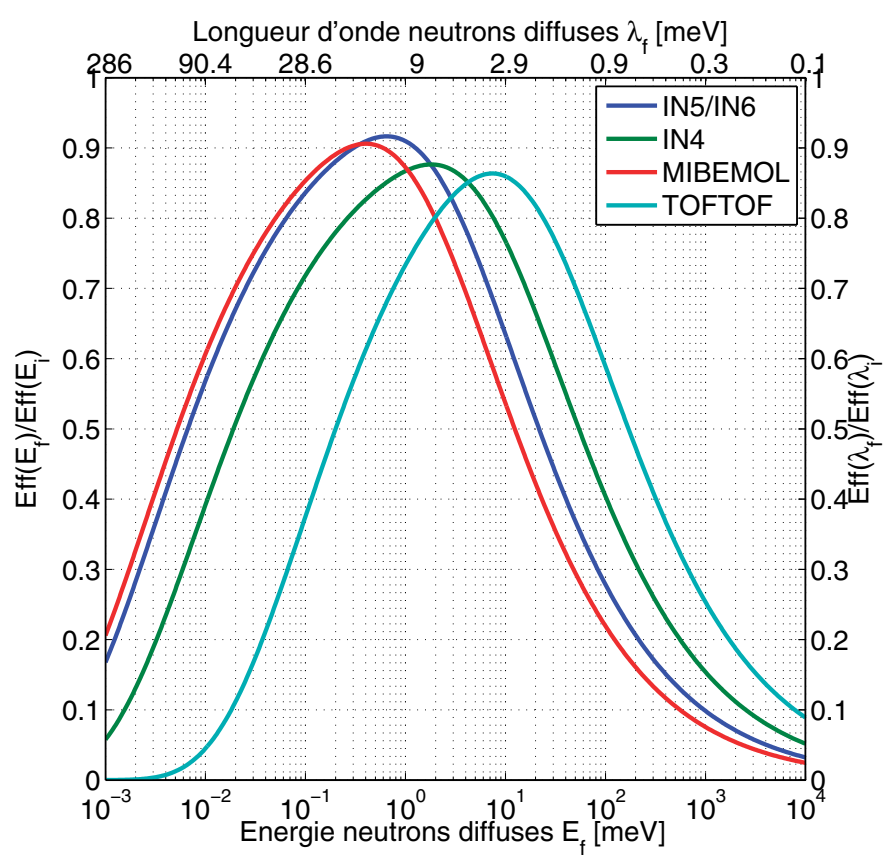

Figure 9. Courbes d'efficacités des détecteurs en fonction de l'énergie des neutrons diffusés pour quelques instruments d'après la formule 4.4.

\subsection{Corrections liées à l'échantillon}

$\rightarrow$ Absorption et auto-écrantage $S_{A F}\left(\phi, \lambda_{f}\right)$ : La répartition en énergie et en angle de diffusion (ou en vecteur de diffusion) est affectée par l'absorption et la diffusion par l'échantillon; elle est ainsi corrélée à sa géométrie.

Les corrections de Sears [19] permettent de calculer l'absorption et l'auto-écrantage pour les géométries simples mais dans ce cas le terme croisé échantillon - cellule n'est pas traité : la diffusion par le porte-échantillon influence la diffusion de l'échantillon, ce qui rend approximative la simple soustraction de la cellule vide.

En pratique cependant, pour un diffuseur suffisamment fort par rapport à la cellule vide, on peut se contenter de la soustraction pondérée de la cellule vide puis d'appliquer les corrections de Sears au premier ordre (première diffusion). On pallie partiellement à l'imperfection de cette méthode pour des échantillons plats en choisissant un angle entre la normale à l'échantillon et l'axe du faisceau incident $\geq 45^{\circ}$. La diffusion est alors plus uniforme et la soustraction simple de la cellule vide associée aux corrections de Sears reste une approximation suffisante dans la plupart des cas. Un autre calcul permet de corriger l'absorption et l'auto-écrantage en intégrant implicitement le porte échantillon [20]. Il est, à notre connaissance, peu usité pour les temps de vol.

$\rightarrow$ Diffusion multiple : Expérimentalement, si aucune précaution n'est prise, il existe une probabilité non nulle qu'un neutron subisse plusieurs processus de diffusion successifs dans l'échantillon avec à chaque fois un échange d'énergie et de direction : on parle de diffusion multiple. On cherche systématiquement à limiter ce phénomène pour rester dans le cadre des modèles et des développements théoriques sur lesquels s'appuie l'analyse des données. Ils supposent tous la connaissance de l'état du neutron incident (énergie et direction) avant son interaction avec l'échantillon; puis ne considèrent qu'une interaction unique entre le neutron et l'échantillon. 
La solution la plus simple consiste à utiliser un échantillon d'épaisseur, e, petite devant le libre parcours moyen des neutrons $\mu$. Cette grandeur s'estime simplement à partir de la composition isotopique de l'échantillon et des tables de section de diffusion. Ainsi, dans la pratique on choisit e, de façon à obtenir une transmission de $\mathcal{T}=90 \%$ : à l'ordre 0 , la probabilité de double diffusion est $(1-\mathcal{T})^{2}=1 \%$, ce qui est inférieur à la précision de la mesure.

Si l'utilisation d'une transmission faible n'est pas possible, on peut s'affranchir du phénomène durant l'analyse des données. Historiquement, pour pallier la faible luminosité des premières sources, on utilisait des échantillons épais si bien que le problème de la diffusion multiple est apparu tôt [21,22]. Des traitements analytiques ont alors été développés, par Sears [19] notamment, puis raffinés par d'autres auteurs [26]). Des traitements par méthode Monte-Carlo basées sur MSC [23], MSCAT [24], DISCUS [25] sont également désormais utilisables.

\subsection{Du nombre "d'événements détecteurs" au facteur de structure dynamique}

Considérons un échantillon $S$ illuminé par un flux $\Phi\left(\lambda_{i}\right)=\frac{d \Phi}{d \lambda_{i}}$ et un détecteur couvrant un angle solide $\Delta \Omega$. Le nombre de neutrons diffusés détectés dans un canal en temps de largeur $\Delta \tau$, sur un intervalle de temps $\mathbb{T}$, correspondant à une "frame" (approximativement une période du chopper le plus lent), est donné par :

$$
\Delta n_{S}=\Phi\left(\lambda_{i}\right) \mathbb{T} \times \epsilon^{S}\left(\phi, \lambda_{f}\right) S_{A F}^{S}\left(\phi, \lambda_{f}\right)\left[\frac{d^{2} \sigma}{d \Omega d t}\right]_{S} \Delta \Omega \Delta \tau
$$

D’après la section de diffusion (Eq. (3.9)) :

$$
\Delta n_{S}=\Phi\left(\lambda_{i}\right) \mathbb{T} \times \epsilon^{S}\left(\phi, \lambda_{f}\right) S_{A F}^{S}\left(\phi, \lambda_{f}\right) \frac{N_{S} \sigma_{S}}{h \sqrt{E_{i}}}\left(\frac{m_{n}}{2}\right)^{3 / 2} \frac{L_{S D}^{3}}{t^{4}} S_{S}(\phi, \omega) \Delta \Omega \Delta \tau
$$

La fonction de correction d'efficacité $\epsilon\left(\phi, \lambda_{f}\right)$ en fonction de l'angle de diffusion et de l'énergie diffusée $\left(E_{f} \propto 1 / \lambda_{f}^{2}\right)$ peut se découpler en un terme dépendant de l'angle de diffusion à énergie incidente fixe, $\epsilon\left(\phi, \lambda_{i}\right)$ (Eq. 4.2) et un terme dépendant de l'énergie quelque soit l'angle de diffusion $\epsilon\left(\lambda_{f} / \lambda_{i}\right)$ (Éq. 4.4) :

$$
\epsilon\left(\phi, \lambda_{f}\right)=\epsilon\left(\phi, \lambda_{i}\right) \times \epsilon\left(\lambda_{f} / \lambda_{i}\right)
$$

La fonction $\epsilon\left(\phi, \lambda_{i}\right)$ n'est pas connue à priori, au contraire de $\epsilon\left(\lambda_{f} / \lambda_{i}\right)$. C'est pourquoi pour chaque expérience il faut faire la mesure d'un échantillon vanadium de référence ayant la même forme géométrique que l'échantillon étudié (voir plus haut).

Reprenant le raisonnement aboutissant à l'équation (4.5) pour le vanadium on obtient :

$$
\Delta n_{V}=\Phi\left(\lambda_{i}\right) T \times \epsilon^{V}\left(\phi, \lambda_{f}\right) S_{A F}^{V}\left(\phi, \lambda_{f}\right)\left[\frac{d^{2} \sigma}{d \Omega d t}\right]_{V} \Delta \Omega \Delta t
$$

avec :

$$
\left[\frac{d^{2} \sigma}{d \Omega d t}\right]_{V}=\frac{N_{V} \sigma_{V}}{h \sqrt{E_{i}}}\left(\frac{m_{n}}{2}\right)^{3 / 2} \frac{L_{S D}^{3}}{t^{4}} S_{V}(\phi, \omega=0)
$$

où $S_{V}(\phi, \omega=0)$ est donné par l'Eq. (4.2).

Combinant les équations (4.5) à (4.9) de façon à séparer les termes mesurés ou calculés (à droite de l'égalité) des termes qui ne le sont pas (à gauche) :

$$
\epsilon\left(\phi, \lambda_{i}\right) \frac{N_{S} \sigma_{S}}{4 \pi} S_{S}(\phi, \omega) \Delta \Omega \Delta t=\frac{\Delta n_{S} t^{4}}{\epsilon\left(\lambda_{f} / \lambda_{i}\right) S_{A F}^{S}\left(\phi, \lambda_{f}\right) 2 E_{i} t_{i}^{3}}
$$




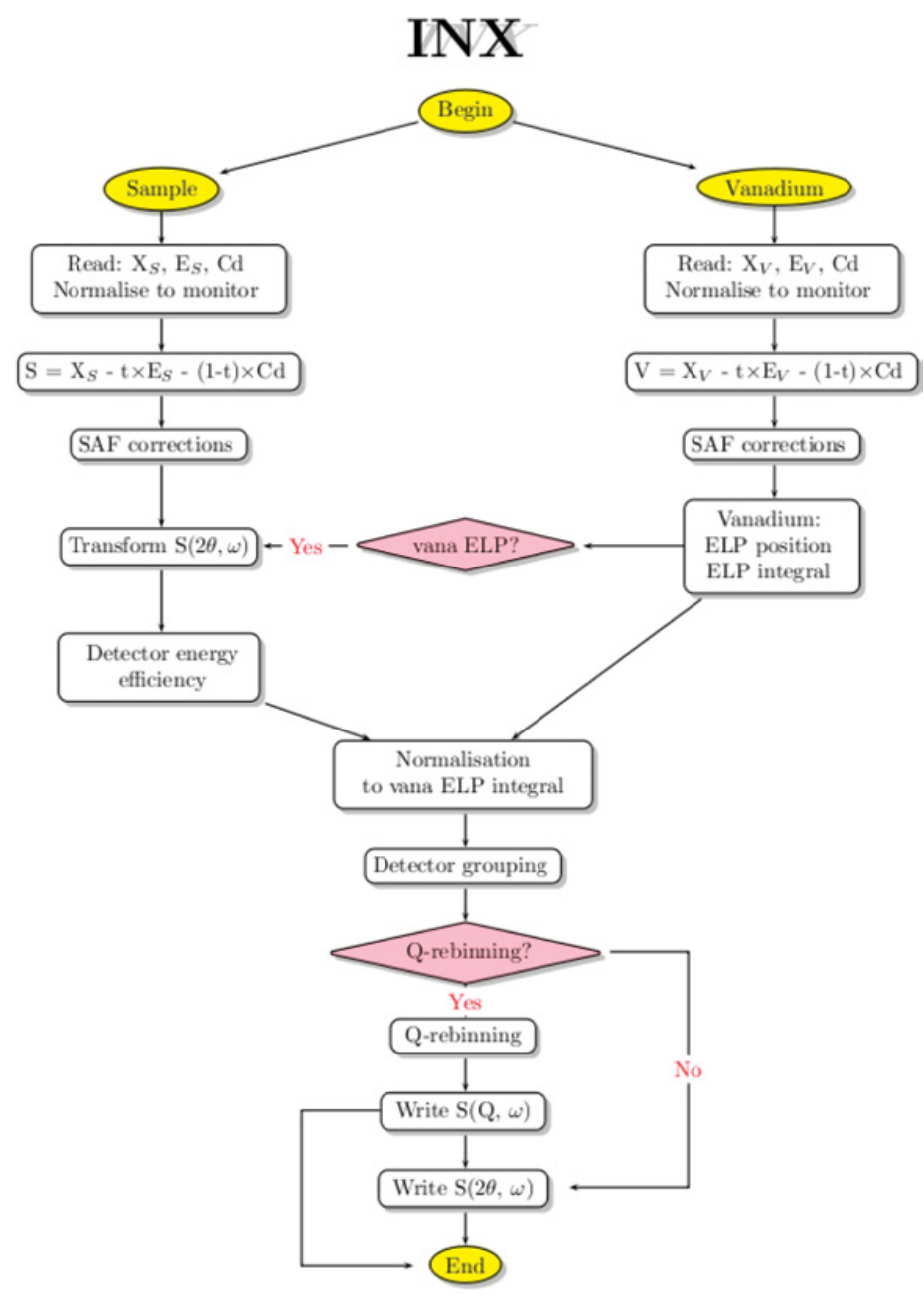

Figure 10. Diagramme de traitement des données temps de vol tel qu'implémenté dans le programme INX à l'ILL.

$$
\epsilon\left(\phi, \lambda_{i}\right) \frac{N_{V} \sigma_{V}}{4 \pi} \Delta \Omega \Delta t=\frac{\Delta n_{V} t_{i}^{4}}{S_{A F}^{S}\left(\phi, \lambda_{i}\right) e^{\left(-\left\langle u_{V}^{2}\right\rangle Q_{e l}^{2}\right)} 2 E_{i} t_{i}^{3}}
$$

Le rapport des termes de l'Éq. (4.10) et (4.11) met en évidence le facteur de structure dynamique :

$$
\frac{N_{S} \sigma_{S}}{N_{V} \sigma_{V}} S_{S}(\phi, \omega)=\frac{\Delta n_{S} S_{A F}^{V}\left(\phi, \lambda_{i}\right) t^{4} e^{\left(-\left\langle u_{V}^{2}\right\rangle Q_{e l}^{2}\right)}}{\Delta n_{V} S_{A F}^{S}\left(\phi, \lambda_{f}\right) t_{i}^{4}}
$$

On obtient en pratique $S_{S}(\phi, \omega)$ multiplié par le rapport des sections efficaces de diffusion totale de l'échantillon et du vanadium de référence. La connaissance du nombre de diffuseurs total du vanadium vus par le faisceau $N_{V}$ permet de remonter à la section efficace absolue de l'échantillon.

L'ensemble des étapes décrites ci-dessus est effectuée dans des programmes de traitement des données tel que INX à l'ILL[18]. L'échantillon et le vanadium sont généralement traités séparément et la normalisation par le terme du vanadium est faite avant les éventuels regroupements de détecteurs ou l'interpolation à vecteur d'onde de transfert $Q$ constant. La figure 10 donne le diagramme de traitement du programme INX utilisé à l'ILL pour des données standard. 
Puisque pour la transformation de l'échelle en énergie on se réfère au pic de diffusion élastique, dans les cas ou l'échantillon ne présente pas de pic élastique bien caractérisé, on utilise celui du vanadium. Le spectre du vanadium donnera également la forme de la résolution expérimentale à transfert d'énergie nul. Elle pourra être convoluée aux modèles théoriques pour permettre la confrontation directe avec les résultats expérimentaux.

\section{LES DIFFÉRENTS TYPES DE SPECTROMÈTRES À TEMPS DE VOL}

On peut classifier les spectromètres à temps de vol selon la méthode utilisée pour le filtrage des neutrons avant échantillon (neutrons incidents) et l'analyse des neutrons diffusés. On distingue trois grandes familles :

- le spectromètre "temps de vol - temps de vol" (ou simplement temps de vol). La sélection de la longueur d'onde incidente se fait par la méthode du temps de vol, typiquement à l'aide de choppers. L'analyse se fait également par temps de vol par enregistrement des neutrons selon leur instant d'arrivée sur le détecteur,

- "temps de vol hybride à géométrie directe". La sélection de la longueur d'onde incidente se fait par diffraction sur monocristal (monochromateur) et l'analyse par mesure du temps de vol.

- "temps de vol hybride à géométrie inverse". La sélection de la longueur d'onde incidente se fait par temps de vol, temps d'arrivée à l'échantillon et l'analyse par diffraction sur monocristal (analyseur). On parle aussi "spectromètres à rétrodiffusion à temps de vol". Cette dernière catégorie ne recouvre que les spectromètres hybrides en mode "rétrodiffusion" (les détecteurs placés derrière l'échantillon sur l'axe échantillon-analyseur) et pas ceux en mode "trois axes" (où les analyseurs sont disposés comme les bras analyseurs de trois axes).

Les instruments appartenant à la première catégorie sont les plus répandus. On en trouve sur source froide sur réacteurs : IN5 (ILL Grenoble), MIBEMOL (LLB Saclay), TOFTOF (BER-II Garching), NEAT (HMI Berlin), DCS (NIST Gaithersburg),...(voir Tableau 2) mais aussi sur source froide sur les sources à spallation : LET (ISIS), CNCS (SNS Oakridge), etc. Technique très appropriée pour les sources à spallation ils se retrouvent aussi sur neutrons thermiques : MERLIN et MAPS (ISIS), ARCS, SEQUOIA (SNS Oakridge), etc, mais pour le moment peu ou pas sur réacteurs bien qu'avec les techniques actuelles il est prouvé qu'ils peuvent y être concurentiels (TOPAS à Garching[15]).

Les instruments tels que IN6, IN4 (ILL Grenoble), FOCUS (PSI Willigen), FCS (NIST Gaithersburg) sont du type hybride à géométrie directe. Ils se retrouvent indifférement sur sources à spallation ou sur réacteurs avec neutrons thermiques ou froids. Ils diffèrent des premiers par le fait que la sélection de la longueur d'onde se fait par monochromateur. Il faut cependant structurer le faisceau, certes monochromatique mais continu, à l'aide d'un (ou plusieurs) choppers (habituellement de Fermi) afin d'amener des pulses monochromatiques au niveau de l'échantillon.

Les instruments hybrides à géométrie inverse sont moins courants en europe : IRIS et OSIRIS (ISIS) sont en mode rétrodiffusion (l'angle de diffusion $\phi=2 \theta$ sur les analyseurs égal à $180^{\circ}$ ). On en retrouve plusieurs au Japon : LAM-80, DNA (J-PARC Tsukuba), etc. Les instruments hybrides à géométrie inverse en mode trois axes (soit l'angle analyseur $<180^{\circ}$ ) sont peu nombreux : PRISMA (ISIS), QENS (en cours de démantèlement à IPNS/Argonne), LAM-40 (J-PARC Tsukuba). Le dernier né est en europe sur source à spallation à flux continu : MARS (PSI, Willigen), etc. Cette fois, le temps initial de départ des neutrons est donné au niveau de la source des neutrons au moment de la création du pulse de neutrons sur la cible de la source à spallation ou à l'aide de choppers près de celle-ci (aussi nécessaire sur source continue). Le pulse polychromatique s'étale entre la source et l'échantillon. L'analyse se fait en enregistrant en temps les neutrons ayant été réfléchis par les analyseurs : pour chaque neutron d'énergie incidente donnée ayant interagi avec l'échantillon seuls les neutrons ayant un transfert d'énergie égal à la différence entre l'énergie incidente et l'énergie permise par l'analyseur sont comptés. Il faut normaliser par le moniteur pour chaque intervalle de temps car le pulse initial n'a aucune raison d'avoir une intensité constante (voir aussi section 5.4). Ce type de spectromètre est bien adapté aux 
Tableau 2. Résumé de la géométrie et du type de source neutronique, des gammes d'énergies et résolutions couvertes pour quelques un des spectromètres à temps de vol les plus connus.

\begin{tabular}{|c|c|c|c|c|c|c|}
\hline Institution & Instrument & Énergie incidente & Géométrie & Source & Gamme d'énergie & Gamme de résolution \\
\hline ISIS/RAL & $\begin{array}{l}\text { HET } \\
\text { MAPS } \\
\text { MARI } \\
\text { PRISMA } \\
\text { MERLIN } \\
\text { LET } \\
\text { IRIS } \\
\text { OSIRIS }\end{array}$ & $\begin{array}{l}15 \mathrm{meV}-2000 \mathrm{meV} \\
15 \mathrm{meV}-2000 \mathrm{meV} \\
10 \mathrm{meV}-1000 \mathrm{meV} \\
10 \mathrm{meV}-1000 \mathrm{meV} \\
1 \mathrm{meV}-80 \mathrm{meV} \\
0.25 \mathrm{meV}-20 \mathrm{meV} \\
0.25 \mathrm{meV}-20 \mathrm{meV}\end{array}$ & $\begin{array}{l}\text { Directe } \\
\text { Directe } \\
\text { Directe } \\
\text { Inverse } \\
\text { Directe } \\
\text { Directe } \\
\text { Inverse } \\
\text { Inverse }\end{array}$ & Spallation & $\begin{array}{l}-\infty>\mathrm{E}_{f}<0.8 \mathrm{E}_{i} \mathrm{meV} \\
-\infty<\mathrm{E}_{f}<0.8 \mathrm{E}_{i} \mathrm{meV} \\
-\infty<\mathrm{E}_{f}<0.8 \mathrm{E}_{i} \mathrm{meV} \\
3 \mathrm{meV}<\mathrm{E}_{f}<20 \mathrm{meV} \\
-\infty<\mathrm{E}_{f}<0.8 \mathrm{E}_{i} \mathrm{meV} \\
-\infty<\mathrm{E}_{f}<0.6 \mathrm{E}_{i} \mathrm{meV} \\
-\infty<\mathrm{E}_{f}<0.6 \mathrm{E}_{i} \mathrm{meV} \\
-0.8<\mathrm{E}_{f}<2.2 \mathrm{meV} \\
-0.5<\mathrm{E}_{f}<0.5 \mathrm{meV} \\
-3.5<\mathrm{E}_{f}<4.0 \mathrm{meV}\end{array}$ & $\begin{array}{l}\Delta \mathrm{E} / \mathrm{E}_{i}=1-2 \%(4 \mathrm{~m}) \\
2-3 \%(2.5 \mathrm{~m}) \\
\Delta \mathrm{E} / \mathrm{E}_{i}=2-5 \% \\
\Delta \mathrm{E}^{-} \mathrm{E}_{i}=1-2 \% \\
\Delta \mathrm{E} / \mathrm{E}_{i}=1-2 \% \\
\Delta \mathrm{E} / \mathrm{E}_{i}=2-5 \% \\
5 \mu \mathrm{eV} \text { at } \mathrm{E}_{i}=1 \mathrm{meV} \\
260 \mu \mathrm{eV} \text { at } \\
\mathrm{E}_{i}=20 \mathrm{meV} \\
1,4.5,11,17.5 \\
54.5 \mu \mathrm{eV} \\
25 \mu \mathrm{eV} \\
90 \mu \mathrm{eV}\end{array}$ \\
\hline SNS & $\begin{array}{l}\text { ARCS } \\
\text { SEQUOIA } \\
\text { CNCS } \\
\text { HYSPEC }\end{array}$ & \begin{tabular}{|l}
$30 \mathrm{meV}-2000 \mathrm{meV}$ \\
$30 \mathrm{meV}-2000 \mathrm{meV}$ \\
$0.8 \mathrm{meV}-20 \mathrm{meV}$ \\
$5 \mathrm{meV}-50 \mathrm{meV}$
\end{tabular} & $\mid \begin{array}{l}\text { Directe } \\
\text { Directe } \\
\text { Directe } \\
\text { Directe }\end{array}$ & Spallation & & $\begin{array}{l}\Delta \mathrm{E} / \mathrm{E}_{i}=2-5 \% \\
\Delta \mathrm{E} / \mathrm{E}_{i}=1-5 \% \\
10-500 \mu \mathrm{eV} \\
\Delta \mathrm{E} / \mathrm{E}_{i}=2-15 \%\end{array}$ \\
\hline PSI-SINQ & $\begin{array}{l}\text { FOCUS } \\
\text { MARS }\end{array}$ & $\begin{array}{l}3 \mathrm{meV}-30 \mathrm{meV} \\
3 \mathrm{meV}-30 \mathrm{meV}\end{array}$ & $\begin{array}{l}\text { Directe } \\
\text { Inverse }\end{array}$ & $\begin{array}{l}\text { Spallation } \\
\text { continue }\end{array}$ & $-\infty<\mathrm{E}_{f}<0.6 \mathrm{E}_{i} \mathrm{meV}$ & $\begin{array}{l}7 \mu \mathrm{eV}<\Delta \mathrm{E}<5 \mathrm{meV} \\
1 \mu \mathrm{eV}<\Delta \mathrm{E} \\
<170 \mu \mathrm{eV}\end{array}$ \\
\hline $\begin{array}{l}\text { LLB } \\
(\mathrm{CEA}-\mathrm{CNRS})\end{array}$ & MIBEMOL & $0.8 \mathrm{meV}-20 \mathrm{meV}$ & Directe & Réacteur & $-\infty<\mathrm{E}_{f}<0.6 \mathrm{E}_{i} \mathrm{meV}$ & $\Delta \mathrm{E} / \mathrm{E}_{i}=1-8 \%$ \\
\hline HMI-BENSC & NEAT & $0.25 \mathrm{meV}-25 \mathrm{meV}$ & Directe & Réacteur & $-\infty<\mathrm{E}_{f}<0.6 \mathrm{E}_{i} \mathrm{meV}$ & $\begin{array}{l}6 \mu \mathrm{eV}<\Delta \mathrm{E} \\
<5.4 \mathrm{meV}\end{array}$ \\
\hline ILL & $\begin{array}{l}\text { IN4 } \\
\text { IN5 } \\
\text { IN6 }\end{array}$ & \begin{tabular}{|l|}
$15 \mathrm{meV}-80 \mathrm{meV}$ \\
$0.2 \mathrm{meV}-20 \mathrm{meV}$ \\
$2.35 \mathrm{meV}-4.8 \mathrm{meV}$
\end{tabular} & \begin{tabular}{|l} 
Directe \\
Directe \\
Directe
\end{tabular} & Réacteur & $\begin{array}{l}-\infty<\mathrm{E}_{f}<0.8 \mathrm{E}_{i} \mathrm{meV} \\
-\infty<\mathrm{E}_{f}<0.6 \mathrm{E}_{i} \mathrm{meV} \\
-\infty<\mathrm{E}_{f}<0.6 \mathrm{E}_{i} \mathrm{meV}\end{array}$ & $\begin{array}{l}\Delta \mathrm{E} / \mathrm{E}_{i}=3-6 \% \\
\Delta \mathrm{E} / \mathrm{E}_{i}=1-3 \% \\
50,80,120,170 \mu \mathrm{eV}\end{array}$ \\
\hline FRM-II & $\begin{array}{l}\text { TOFTOF } \\
\text { TOPAS }\end{array}$ & $\begin{array}{l}0.3 \mathrm{meV}-20 \mathrm{meV} \\
20 \mathrm{meV}-160 \mathrm{meV}\end{array}$ & $\begin{array}{l}\text { Directe } \\
\text { Directe }\end{array}$ & $\begin{array}{l}\text { Réacteur } \\
\text { Réacteur }\end{array}$ & $-\infty<\mathrm{E}_{f}<0.6 \mathrm{E}_{i} \mathrm{meV}$ & $\begin{array}{l}\Delta \mathrm{E} / \mathrm{E}_{i}=1-3 \% \\
\Delta \mathrm{E} / \mathrm{E}_{i}=5 \%\end{array}$ \\
\hline IPNS, ANL & $\begin{array}{l}\text { HRMECS } \\
\text { LRMECS } \\
\text { QENS } \\
\end{array}$ & $\begin{array}{l}3 \mathrm{meV}-1000 \mathrm{meV} \\
6 \mathrm{meV}-600 \mathrm{meV} \\
?\end{array}$ & \begin{tabular}{|l} 
Directe \\
Directe \\
Inverse
\end{tabular} & Spallation & $\begin{array}{l}0-800 \mathrm{meV} \\
0-500 \mathrm{meV} \\
-2.5<\mathrm{E}_{f}<200 \mathrm{meV}\end{array}$ & $\begin{array}{l}\Delta \mathrm{E} / \mathrm{E}_{i}=2-4 \% \\
\Delta \mathrm{E} / \mathrm{E}_{i}=6-8 \% \\
90 \mu \mathrm{eV}\end{array}$ \\
\hline NIST, NCNR & $\begin{array}{l}\text { FCS } \\
\text { DCS }\end{array}$ & \begin{tabular}{|l|}
$2.2 \mathrm{meV}-15 \mathrm{meV}$ \\
$0.4 \mathrm{meV}-13 \mathrm{meV}$
\end{tabular} & $\begin{array}{l}\text { Directe } \\
\text { Directe }\end{array}$ & Réacteur & $\mid \begin{array}{l}-\infty<\mathrm{E}_{f}<0.6 \mathrm{E}_{i} \mathrm{meV} \\
-\infty<\mathrm{E}_{f}<0.6 \mathrm{E}_{i} \mathrm{meV}\end{array}$ & $\begin{array}{l}60 \text { to } 1000 \mu \mathrm{eV} \\
\Delta \mathrm{E} / \mathrm{E}_{i}=1-3 \%\end{array}$ \\
\hline $\begin{array}{l}\text { J-PARC } \\
\text { KENS/MLF } \\
\text { KEK } \\
\text { KENS } \\
\text { Tsukuba }\end{array}$ & $\begin{array}{l}\text { CNDCS } \\
\text { LAM-D } \\
\text { LAM-40 } \\
\text { LAM-80 } \\
\\
\text { AMATERA } \\
\text { 4SEASONS } \\
\text { DNA } \\
\text { HRC }\end{array}$ & $\begin{array}{l}1 \mathrm{meV}-80 \mathrm{meV} \\
1 \mathrm{meV}-60 \mathrm{meV} \\
1 \mathrm{meV}-60 \mathrm{meV} \\
1 \mathrm{meV}-60 \mathrm{meV} \\
\\
1 \mathrm{meV}-80 \mathrm{meV} \\
\\
5 \mathrm{meV}-300 \mathrm{meV} \\
1 \mathrm{meV}-60 \mathrm{meV} \\
1 \mathrm{meV}-2000 \mathrm{meV}\end{array}$ & $\begin{array}{l}\text { Directe } \\
\text { Inverse } \\
\text { Inverse } \\
\text { Inverse } \\
\\
\text { Directe } \\
\text { Directe } \\
\text { Inverse } \\
\text { Directe }\end{array}$ & \begin{tabular}{|l} 
Spallation \\
Spallation
\end{tabular} & $\begin{array}{l}-2<E_{f}<60 \mathrm{meV} \\
-2<E_{f}<10 \mathrm{meV} \\
-30<\mathrm{E}_{f}<30 \mu \mathrm{eV} \\
-400<\mathrm{E}_{f}<500 \mu \mathrm{eV} \\
-1<\mathrm{E}_{f}<1.5 \mathrm{meV}\end{array}$ & $\begin{array}{l}\Delta \mathrm{E} / \mathrm{E}_{i} \simeq 1 \% \\
\left(\mathrm{E}_{i}=20 \mathrm{meV}\right) \\
350 \mu \mathrm{eV} \\
200 \mu \mathrm{eV} \\
1.5 \mu \mathrm{eV} \\
6.5 \mu \mathrm{eV} \\
17 \mu \mathrm{eV} \\
\Delta \mathrm{E} / \mathrm{E}_{i}=1-7 \% / \\
0.3-1.5 \% \\
\Delta \mathrm{E} / \mathrm{E}_{i}=6 \% \\
\Delta \mathrm{E}=1 \mu \mathrm{eV} / 10 \mu \mathrm{eV} \\
\Delta \mathrm{E} / \mathrm{E}_{i}=1 \%\end{array}$ \\
\hline
\end{tabular}

sources à spallation pulsées dont ils tirent bénéfice, entre autres, du faible taux de répétition des pulses. Il est cependant possible d'en construire sur les sources continues (à spallation ou réacteur). Dans ce cas un système de choppers sert à créer les pulses (MARS sur la source à spallation continue du PSI, Willigen, Suisse). 

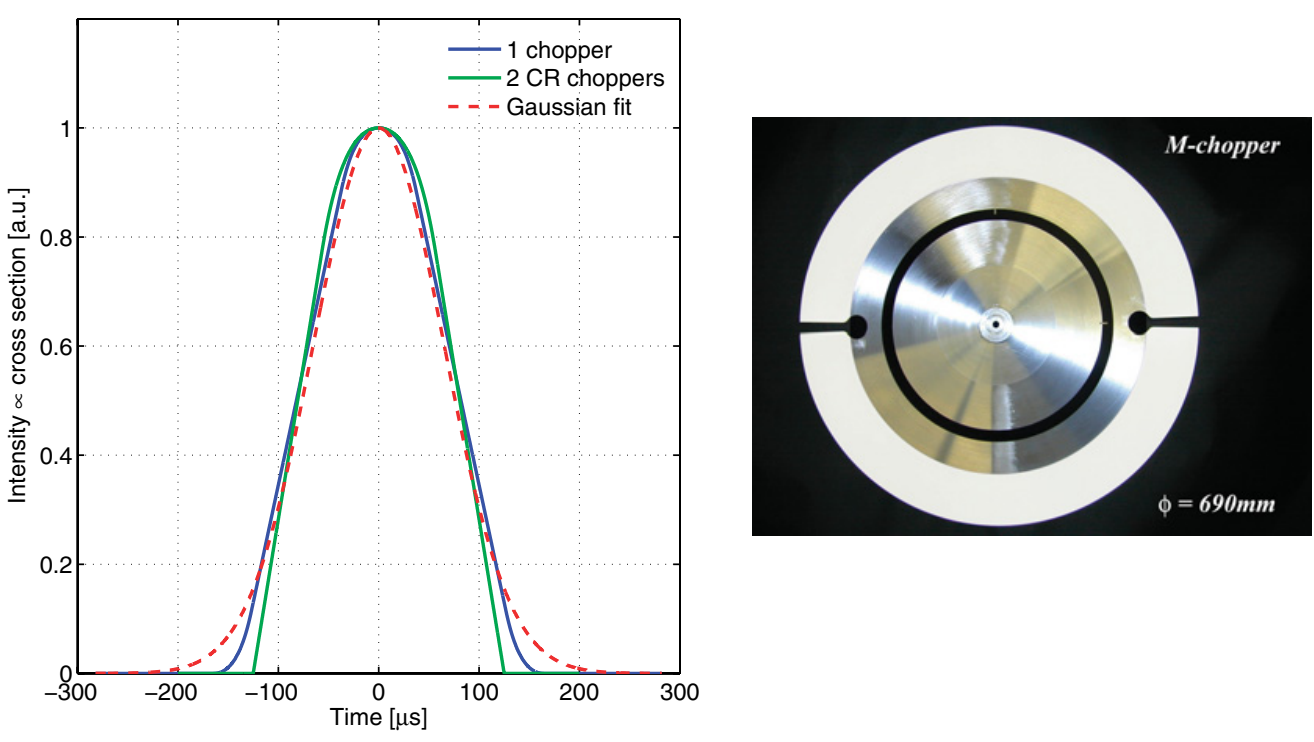

Figure 11. Gauche : Formes théoriques des pulses de neutrons passant par le guide au travers d'un disque et de 2 disques en contre rotation (counter rotating choppers - CRC). Le pied du pic élastique comporte moins "d'ailes". Droite : disque en aluminium pour la dernière paire de choppers sur IN5. La partie blanche est de l'oxyde de gadolinium, un absorbeur de neutrons.

\subsection{Le spectromètre temps de vol - temps de vol}

C'est le type de spectromètre le plus ancien dans le concept (voir section 5). L'instrument IN5 fut le premier temps de vol construit à l'ILL (opérationel dès début 1973) d'après une étude de R. Scherm et T. Springer [27]. L'idée était de faire un instrument permettant un accès à de très hautes résolutions (à l'époque) couplé à une grande flexibilité longueur d'onde / vecteur d'onde de transfert / résolution. Le schéma proposé était composé de 2 choppers pour la monochromatisation (premier et derniers), de 1 ou 2 choppers pour éviter les longueurs d'onde "harmoniques" et d'un chopper pour éviter le recouvrement des spectres dans certaines configurations. Ce concept est resté inchangé depuis. Il a cependant été perfectionné par le remplacement des choppers individuels de monochromatisation par des couples de chopper en rotation inverses (counter-rotating) : les choppers d'un même couple sont composés de 2 disques identiques placés aussi près que possible l'un de l'autre et ayant des sens de rotation opposés. Ils permettent :

- d'obtenir des pulses plus courts à vitesse égale car ils sont équivalents à un seul chopper tournant 2 fois plus vite.

- de symétriser la structure temporelle du pulse.

- d'améliorer la forme de la résolution i.e. l'obtention d'une meilleure forme que la forme triangulaire (comme avec un seul chopper, Fig. 11). La forme de la résolution s'ajuste très bien avec une gaussienne de largeur à mi-hauteur $\delta \hbar \omega$. Cette structure "sans ailes" de la résolution permet d'optimiser la détection des signaux relaxationnels qui se manifestent sous la forme de lorentziennes même lorsqu'ils sont peu intenses.

Le nombre de groupes de choppers minimum pour les instruments à chopper à disques est aujourd'hui de $3: 2$ groupes (soit 4 choppers) assurent la monochromatisation et un groupe remplit les fonctions antiharmonique et anti-recouvrement ( 2 choppers). Ceci est vrai si les disques des choppers n'ont qu'une fente (ou une paire de fentes placées symétriquement). C'est le cas de IN5 à l'ILL ou de MIBEMOL 

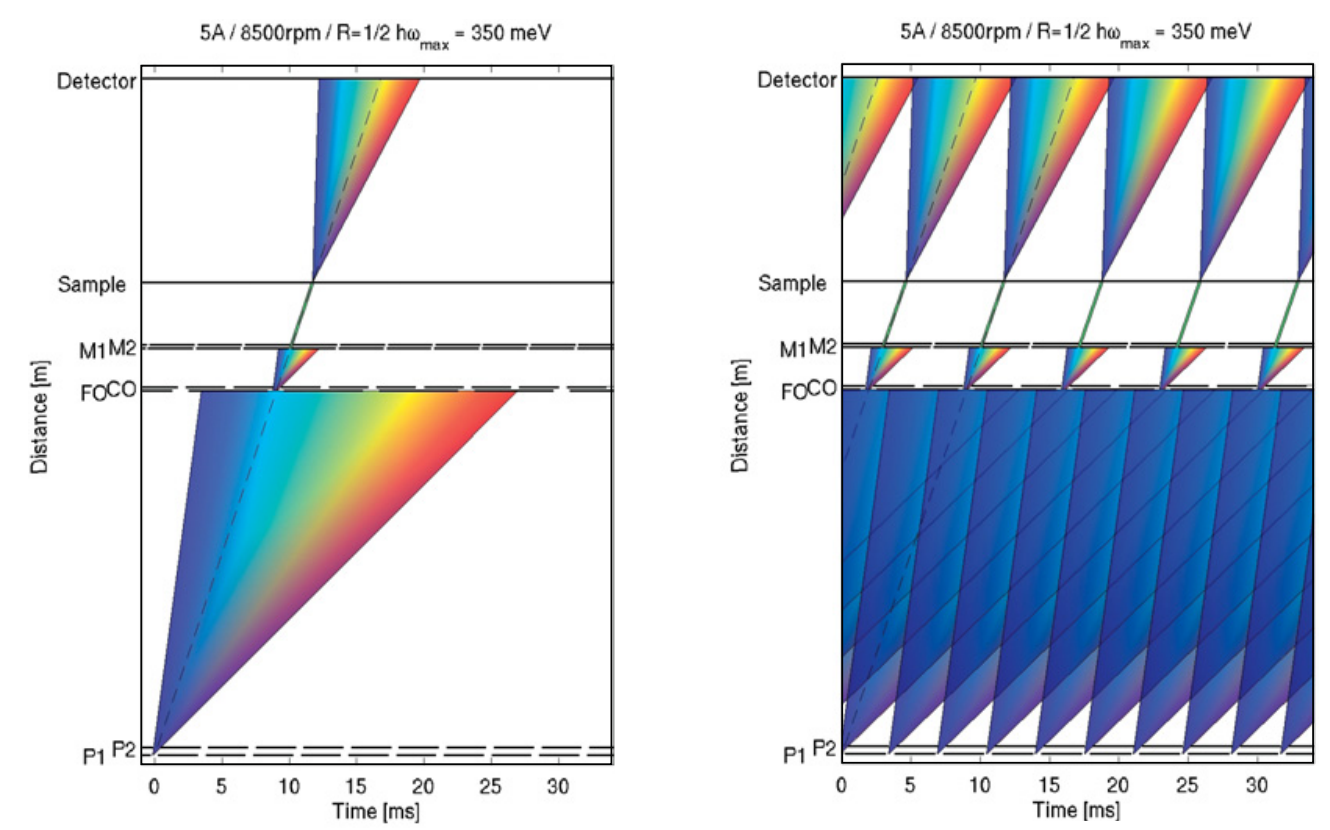

Figure 12. Gauche : Diagramme temps-distance pour IN5 pour un pulse de neutrons (paramètres spécifiés audessus du schéma). Les couleurs donnent une indication de l'énergie des neutrons : du bleu (plus) au rouge (moins) énergétiques. Droite : Idem pour le ratio spécifié $R=1 / 2:$ la fenêtre en énergie permet juste de ne pas avoir de recouvrement sans perdre de temps de faisceau.

au LLB. Pour les instruments qui présentent plusieurs types de fentes sur les disques, il faut rajouter un $4^{\text {ème }}$ groupe anti-harmonique, par exemple : NEAT au HMI, DCS au NIST et TOFTOF à FRM-II.

\section{Rôle des différents disques}

La première paire de disques (Pulsing choppers - P) crée un pulse "blanc", de distribution des vitesses approximativement maxwellienne. Cette bouffée se disperse le long du guide avant d'atteindre la dernière paire de disques (choppers de Monochromatisation - M) située à une distance $L_{12}$, après un temps $t_{12}$. La longueur d'onde finale $\lambda_{i}$ est déterminée par le choix du déphasage temporel $t_{12}$ entre les deux couples de choppers (voir figure 12).

L'étalement temporel des neutrons sur plusieurs mètres peut entraîner le recouvrement de deux bouffées successives : les neutrons les plus rapides du pulse $\mathrm{N}+1$ doublant les plus lents du pulse $\mathrm{N}$. Pour éviter une trop grande dispersion, la bande en longueur d'onde est réduite par le $2^{\text {ème }}$ groupe de choppers (figure 12). Sur IN5, les disques de la première paire sont aussi plus écartés (15 cm au lieu du minimum de $5 \mathrm{~cm}$ pour les autres) afin d'améliorer cette filtration. Les neutrons, monochromatisés par la dernière paire de choppers, arrivant sur l'échantillon vont se transformer en une large bande de longueur d'onde au niveau des détecteurs après s'être dispersés dans la chambre temps de vol suite à l'échange d'énergie avec l'échantillon. Afin d'éviter le recouvrement des pulses successifs au niveau des détecteurs, un disque supplémentaire tournant à une vitesse moins grande que les autres choppers, le chopper d'antirecouvrement ${ }^{12}$, s'ajoute dans le $2^{\text {ème }}$ groupe (sur IN5). Il permet d'ajuster la séparation en temps des pulses arrivant sur l'échantillon (figure 12).

Le ratio $R=V_{C H} / V_{F O}$ des vitesses entre le chopper d'anti-recouvrement et les autres est un paramètre important pour optimiser le flux à longueur d'onde incidente et vitesse des choppers donnés.

\footnotetext{
12 FO pour frame overlap dans la nomenclature IN5.
} 


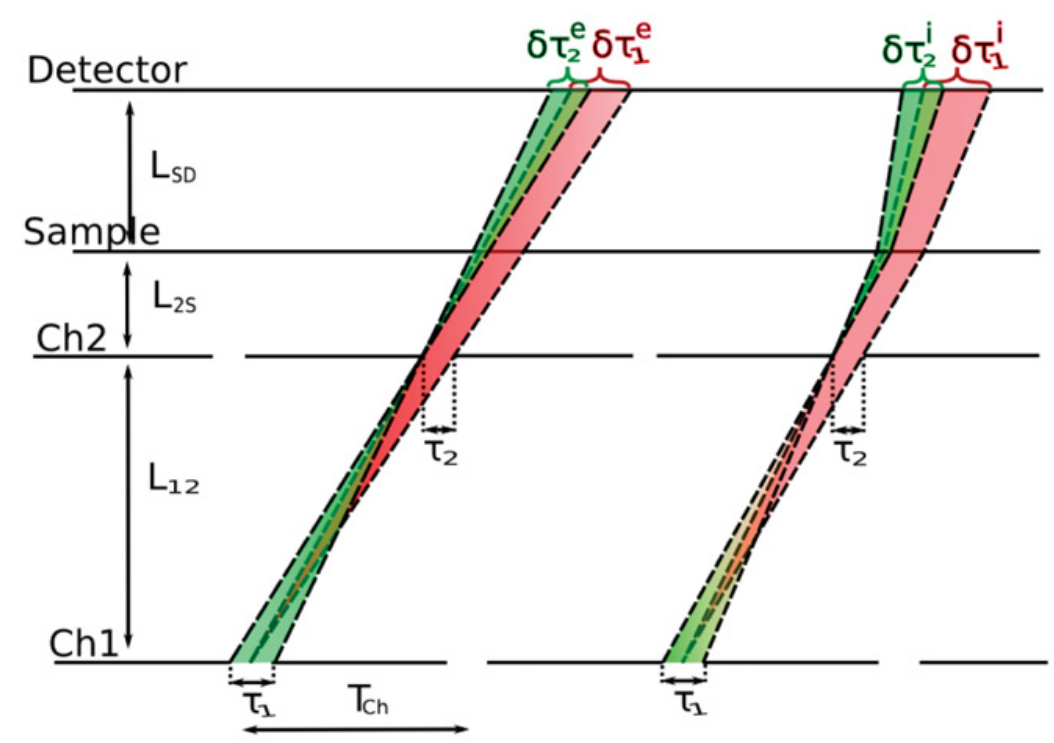

Figure 13. Schéma simplifié définissant les paramètres entrant dans le calcul de l'incertitude due au spectromètre primaire.

\subsubsection{Fonction de résolution en énergie}

Le calcul analytique de la fonction de résolution de ce type de spectromètre a été abordé au paragraphe 3.3. Ce paragraphe se contente d'apporter un raisonnement plus physique sur les incertitudes.

\section{Incertitudes dues au spectromètre primaire}

Dans le calcul suivant, on assimile le premier couple de choppers de fréquence $v$ et $-v$ à un chopper simple de fréquence $2 v$ (chopper 1). Le schéma simplifié de la figure 13 permet de déduire les incertitudes temporelles $\delta \tau_{1}$ et $\delta \tau_{2}$ élastiques puis inélastiques. Les temps d'ouverture des choppers $\tau_{1}$ et $\tau_{2}$ sont égaux à l'angle d'ouverture $\alpha_{i}(\mathrm{i}=1,2)$ divisé par les vitesses angulaires $\omega_{i}=2 \pi v_{i}$ : $\tau_{i}=\alpha_{i} / \omega_{i}$.

Les termes de résolution élastique $\delta \tau_{i}^{e}(\mathrm{i}=1,2)$ se calculent comme la convolution d'un terme d'élargissement $\delta \tau_{1}^{e}$ provenant d'un point de l'ouverture $\tau_{1}$ du chopper 1 et passant par n'importe quel point de l'ouverture $\tau_{2}$ du chopper 2 avec un terme d'élargissement $\delta \tau_{2}^{e}$ provenant de n'importe quel point de l'ouverture du chopper 1 mais passant par un point de l'ouverture du chopper 2. Un simple calcul de géométrie permet de déduire :

$$
\begin{aligned}
& \delta \tau_{1}^{e}=\frac{\left(L_{12}+L_{2 S}+L_{S D}\right)}{L_{12}} \tau_{2} \\
& \delta \tau_{2}^{e}=\frac{\left(L_{2 S}+L_{S D}\right)}{L_{12}} \tau_{1}
\end{aligned}
$$

Les termes de résolution inélastique $\delta \tau_{i}^{i}(i=1,2)$ se définissent de la même façon mais cette fois en faisant intervenir un changement d'énergie $\hbar \omega$ au niveau de l'échantillon. On remarque que les temps de parcours le long du chemin peuvent s'écrire, compte tenu de l'échange d'énergie :

$$
\begin{aligned}
t_{1 D} & =C\left[\left(L_{12}+L_{2 S}\right) \lambda_{i}+L_{S D} \lambda_{f}\right] \\
t_{2 S} & =C\left[L_{2 S} \lambda_{i}+L_{S D} \lambda_{f}\right]
\end{aligned}
$$


On doit alors tenir compte de la dispersion du temps d'arrivée $\delta t \propto \delta \lambda_{f}$ après changement d'énergie compte tenu de la dispersion du temps correspondant aux neutrons incidents $\delta t_{i} \propto \delta \lambda_{i}$ à transfert d'énergie $\hbar \omega$ constante [28]. Différenciant (5.3), la variation de temps d'arrivée due à la dispersion en longueur d'onde $\delta \lambda_{i}$ s'écrira :

$$
\begin{aligned}
& \frac{\delta t_{1 D}}{\delta \lambda_{i}}=C\left[L_{12}+L_{2 S}+L_{S D}\left(\frac{\delta \lambda_{f}}{\delta \lambda_{i}}\right)\right] \\
& \frac{\delta t_{2 S}}{\delta \lambda_{i}}=C\left[L_{2 S}+L_{S D}\left(\frac{\delta \lambda_{f}}{\delta \lambda_{i}}\right)\right]
\end{aligned}
$$

En substituant dans 5.1 et 5.2, compte tenu que :

on obtient :

$$
\frac{\delta \lambda_{f}}{\delta \lambda_{i}}=\left(\frac{\lambda_{f}}{\lambda_{i}}\right)^{3}
$$

$$
\begin{aligned}
& \delta \tau_{1}^{i}=\left[\frac{L_{12}+L_{2 S}+L_{S D}\left(\frac{\lambda_{f}}{\lambda_{i}}\right)^{3}}{L_{12}}\right] \tau_{2} \\
& \delta \tau_{2}^{i}=\left[\frac{\left(L_{2 S}+L_{S D}\left(\frac{\lambda_{f}}{\lambda_{i}}\right)^{3}\right.}{L_{12}}\right] \tau_{1}
\end{aligned}
$$

\section{Incertitudes dues à l'échantillon}

Cette incertitude est essentiellement due à la dispersion sur la distance de vol échantillon-détecteur entre différents points de diffusion dans l'échantillon. On donne, par exemple, la contribution à l'incertitude sur le temps de vol échantillon - détecteur pour un échantillon plaque, géométrie souvent employée sur ces appareils (Fig. 14). Il se décompose en 2 termes[29], un pour une plaque infiniment fine de largeur finie $w$ (ou de façon équivalente supérieure à la largeur du faisceau) :

$$
\delta \tau_{s 1}=C \frac{w \lambda_{i}}{2 \sin \psi}\left[\cos \psi-\frac{\cos (\psi-\Phi)}{\sqrt{1-\hbar \omega / E_{i}}}\right]
$$

et l'autre pour une plaque infiniment large mais dépaisseur finie $e$ :

$$
\delta \tau_{s 2}=0.68 C \frac{e \lambda_{i}}{\sin \psi}\left[1-\frac{\cos \Phi}{\sqrt{1-\hbar \omega / E_{i}}}\right]
$$

que d'aucuns somment en quadrature ${ }^{13}$.

\section{Incertitudes induite par le détecteur}

La capture du neutron diffusé ne se fait pas sur l'anode du détecteur située sur l'axe du tube (Fig. 14) mais à une postion indéterminée dans le gaz absorbeur. Il en résulte une incertitude sur la distance échantillon-détecteur supplémentaire qui dépend aussi de la forme du détecteur (on néglige le temps de dérive des charges entre leur lieu de génération et l'anode où le signal est effectivement détecté). Le barycentre de la localisation de détection dépend de l'énergie du neutron diffusé : plus l'énergie du neutron sera faible, moins la pénétration dans le tube sera grande. En première approximation on maximise cette incertitude en estimant une détection homogène quelque soit l'énergie du neutron :

$$
\delta \tau_{D}=C . D . \lambda_{f}
$$

où $D$ est le diamètre du tube détecteur, typiquement de l'ordre d'un pouce $(2.54 \mathrm{~cm})$.

13 D'autres expressions ont été proposées, par exemple [36]. 

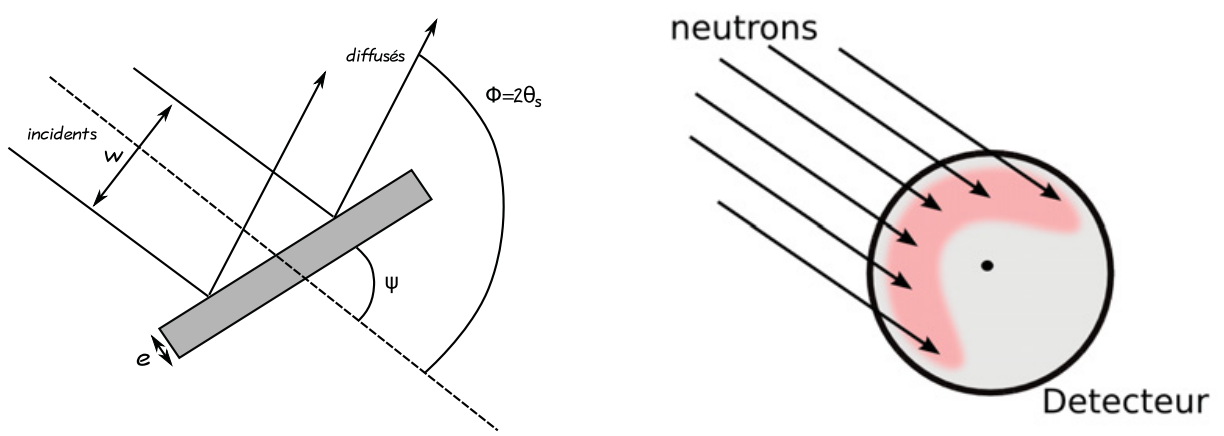

Figure 14. Gauche : Définition des paramètres pour un échantillon sous forme de plaque. $\Phi$ est l'angle de diffusion, $\psi$ est l'orientation de la plaque dans le faisceau incident. Droite: Vue schématique, en coupe perpendiculaire à l'axe de la localisation, de la détection dans un détecteur cylindrique.

Rassemblant les expressions $5.4-5.10$, on obtient une expression pratique, qui s'identifie bien à (3.17), permettant de calculer avec une bonne approximation la résolution en énergie (les temps sont en secondes, les distances en mètres, la longueur d'onde en $\AA$ ) :

$$
\begin{aligned}
\delta \hbar \omega[\mathrm{meV}] & =\frac{0.6472}{L_{S D} \lambda_{f}^{3}} \delta t \\
\delta t^{2} & =\left(\delta \tau_{1}^{i}\right)^{2}+\left(\delta \tau_{2}^{i}\right)^{2}+\delta \tau_{s 1}^{2}+\delta \tau_{s 2}^{2}+\delta \tau_{D}^{2}
\end{aligned}
$$

La résolution en énergie varie linéairement avec les distances et la vitesse des choppers mais avec le cube de la longueur d'onde. Pour faire varier la résolution il faut donc privilégier le changement de longueur d'onde sur le changement de vitesse des choppers (les autres paramètres étant fixes). La figure 15 montre, entre autres, qu'à une longueur d'onde donnée, plus la vitesse des choppers est grande, plus les termes dépendants de l'incertitude sur le temps de vol échantillon-détecteur sont prépondérants.

\subsubsection{Figure d'espace des configurations}

La résolution en énergie dépend donc des paramètres géométriques (des distances : $L_{12}, L_{2 S}, L_{S D}$ et des angles des fentes choppers $\alpha_{1}, \alpha_{2}$ ) combinés aux paramètres "dynamiques" : longueur d'onde incidente $\left(\lambda_{i}\right)$ et vitesse de rotation des choppers $V_{c h}$.

On peut combiner ces paramètres, associés au flux incident avant choppers $\Phi\left(\lambda_{i}\right)$ dans un "espace des configurations" (pour IN5, par exemple, voir Fig. 16). La longueur maximale d'une frame est définie par la période $T[\mathrm{~s}]=\frac{60}{V_{c h}[\mathrm{rpm}]}$ du chopper d'antirecouvrement, donc par le ratio $R$. Cette période est déterminée par le temps nécessaire pour enregistrer tous les neutrons d'un pulse atteignant les détecteurs avant que le pulse suivant n'arrive à l'échantillon (ou, plus précisément, ne commence à se disperser après interaction avec celui-ci). Par une règle empirique ${ }^{14}$, on admet que les derniers neutrons qui arrivent aux détecteurs sont ceux ayant une énergie telle que leur longueur d'onde soit égale à 1.5 fois la longueur d'onde incidente : $\lambda_{f_{\max }} \approx 1.5 \lambda_{i}$. Au-delà il n'y a statistiquement plus assez de neutrons à détecter. Entre deux ratio $R$ l'intensité moyenne varie comme le rapport des vitesses du chopper d'antirecouvrement visualisé par les marches sur les lignes d'iso-intensité. Un point sur la figure 16 définit une configuration : longueur d'onde incidente, vitesse des choppers, ratio, intensité et résolution élastique.

\footnotetext{
14 Cette règle s’applique principalement à la diffusion quasiélastique.
} 

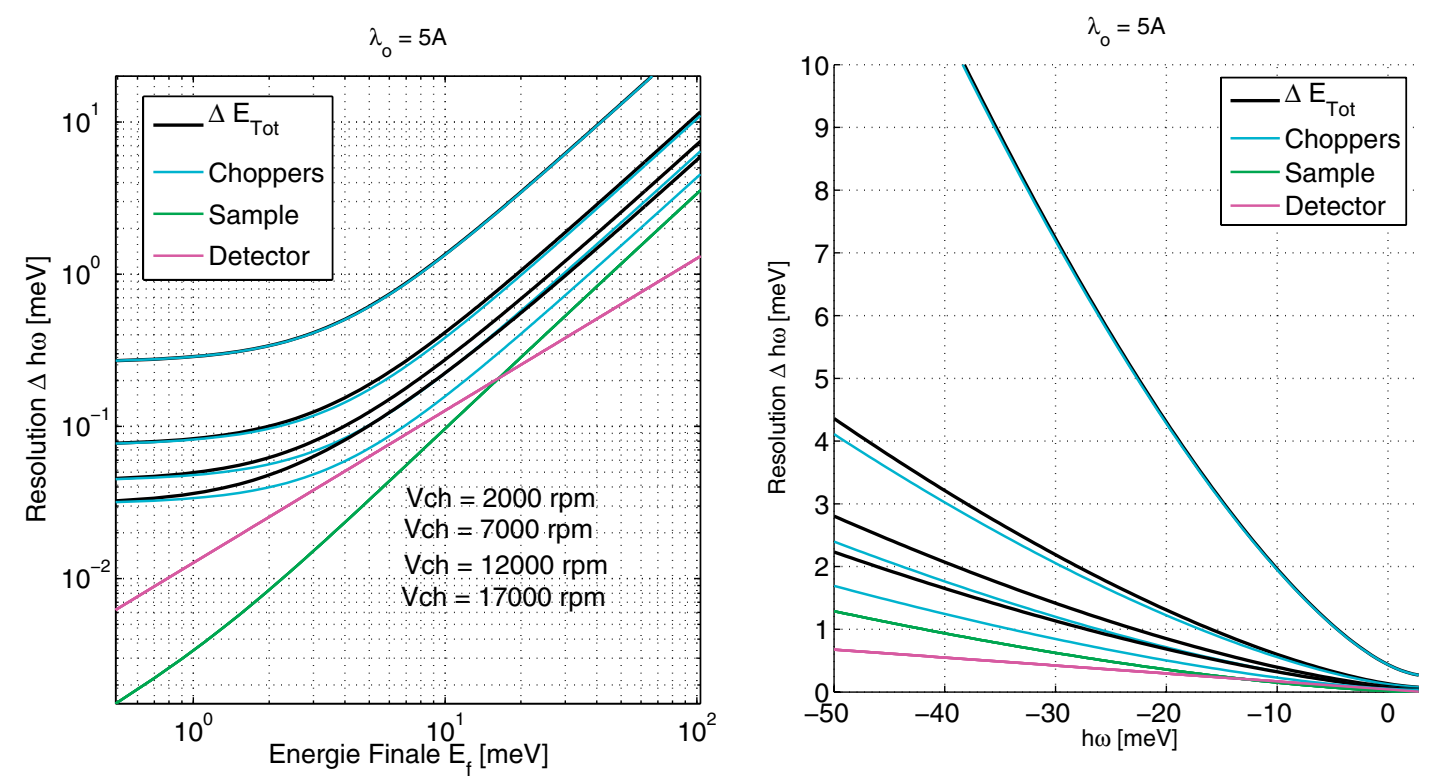

Figure 15. Gauche : Résolution en énergie à longueur d'onde incidente fixe en fonction de l'énergie diffusée (échelle logarithmique) pour quelques vitesses de choppers. Droite : Même figure en fonction du transfert d'énergie (échelle linéaire).

A une longueur d'onde incidente donnée qui définit la gamme en $Q$ accessible et une résolution souhaitée correspond un seul point qui maximise l'intensité sur l'échantillon.

\subsubsection{Optimisation du spectromètre}

Il est frappant de constater que les spectromètres à choppers sur neutrons froids ont tous à peu près les mêmes dimensions, mis à part NEAT (HMI, Berlin). Ceci est dû à l'optimisation de leur géométrie compte tenu de l'utilisation de technologies similaires. L'optimisation consiste à obtenir le meilleur flux possible pour une résolution donnée, ou la meilleure résolution possible pour un flux donné [13].

On peut définir le ratio $\rho$ des pulses aux choppers :

$$
\rho=\frac{\tau_{1}}{\tau_{2}}
$$

L'intensité sur le détecteur, sans tenir compte de l'interaction avec l'échantillon, est proportionnelle au flux incident $\Phi\left(\lambda_{i}\right)$, à la durée d'ouverture des choppers $\tau_{i}=\alpha_{i} / \omega_{i}\left(\alpha_{i}\right.$ et $\omega_{i}$ sont les largeurs de fentes et vitesses angulaires des choppers; $\mathrm{i}=1,2)$ et inversement proportionnelle aux distances $L_{12}$ et $L_{S D}$ :

$$
I \propto \Phi\left(\lambda_{i}\right) \frac{\tau_{1} \tau_{2}}{L_{12} L_{S D}}
$$

Les autres paramètres étant fixés, seul le produit $\tau_{1} \tau_{2}$ peut varier en changeant la vitesse des choppers. On néglige dans le raisonnement les termes dépendants de l'incertitude échantillon et détecteurs :

$$
\delta t^{2}=\left(\delta \tau_{1}^{i}\right)^{2}+\left(\delta \tau_{2}^{i}\right)^{2}
$$




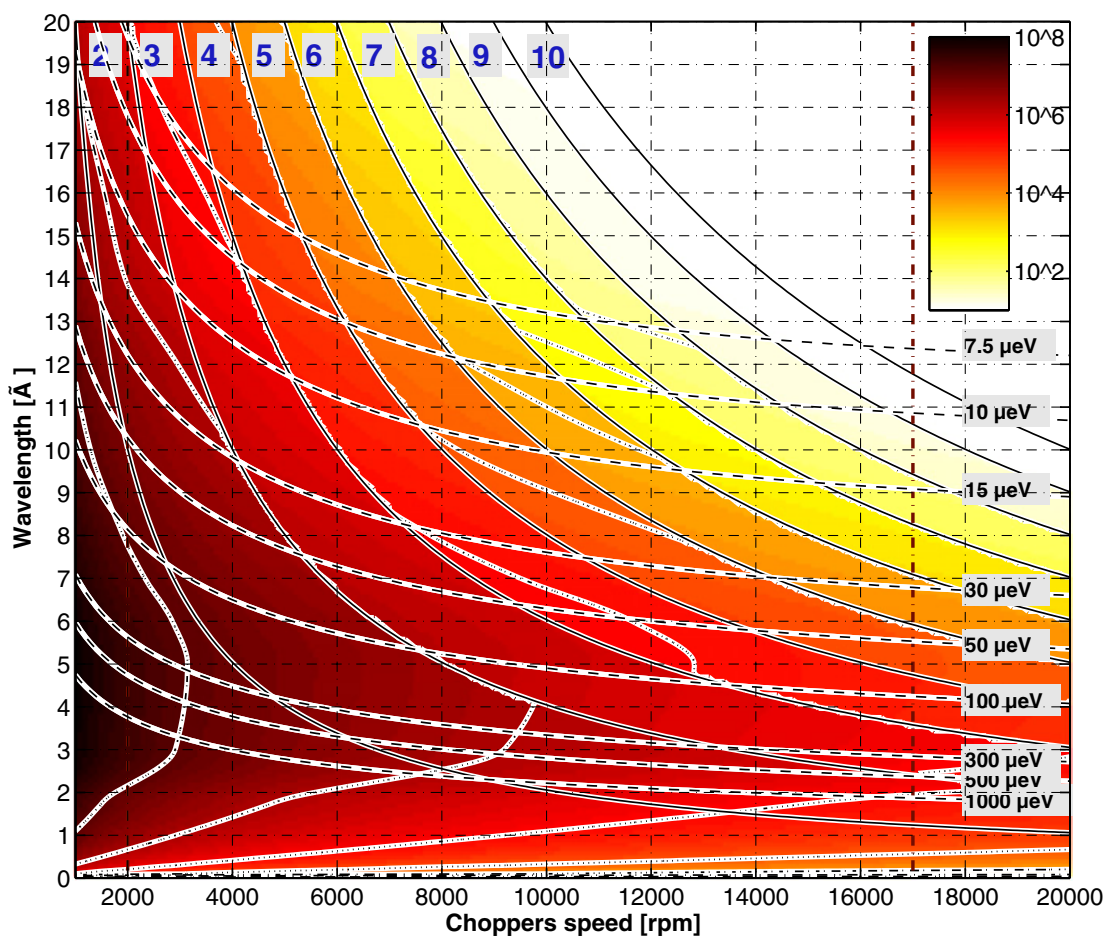

Figure 16. Diagramme multi paramètres (vitesse des choppers, longueur d'onde incidente, intensité, ratio 1/R, résolution) pour un spectromètre temps de vol à disques (ici IN5 à l'ILL). L'intensité est représentée par la charte colorée. Les gammes de ratio $1 / R$, indiquées en haut du schéma, sont représentées en lignes continues. Les lignes pointillées représentent les courbes iso-résolution dont les valeurs sont notées en bout de ligne.

Réécrivant 5.6 et 5.7 sous la forme :

$$
\begin{array}{ll}
\delta \tau_{1}^{i}=\frac{b \tau_{2}}{L_{12}}, & b=L_{12}+L_{2 S}+L_{S D}\left(\frac{\lambda_{f}}{\lambda_{i}}\right)^{3} \\
\delta \tau_{2}^{i}=\frac{c \tau_{1}}{L_{12}}, & c=L_{2 S}+L_{S D}\left(\frac{\lambda_{f}}{\lambda_{i}}\right)^{3}
\end{array}
$$

on peut exprimer le produit $\tau_{1} \tau_{2}$ en fonction de $\rho$ en partant de 5.15 :

$$
\tau_{1} \tau_{2}=\frac{L_{12}^{2} \delta t^{2}}{b^{2} \rho^{-1}+c^{2} \rho}
$$

Cette fonction présente un maximum à résolution $\delta t$ fixée $: \rho_{\mathrm{opt}}=\frac{b}{c}$ correspondant à $\delta \tau_{1}^{i} \approx \delta \tau_{2}^{i 15}$. La figure 17 présente les cas de IN5 (ILL, Grenoble) et NEAT (HMI, Berlin). Le rapport des ouvertures des fentes pour IN5 est de $\alpha_{1} / \alpha_{2}=2.79$, les vitesses des choppers dans la configuration standard avec $\mathrm{V}_{\mathrm{CH}_{1}}=\mathrm{V}_{\mathrm{CH}_{2}}$ sont très proches des valeurs "idéales". Les paramètres géométriques de IN5 sont optimisés pour des vitesses égales des première et dernière paires de choppers.

En revanche, NEAT $\left(\alpha_{1} / \alpha_{2}=2.0\right.$ avec les fentes de largeur égales à la largeur du guide) qui se démarque par le rapport des distances $L_{12} / L_{S D}$ n'est pas optimisé pour des vitesses de choppers égales. On remarque que les valeurs idéales sont proches de $\mathrm{V}_{c h_{1}}=6250 \mathrm{rpm}$ et $\mathrm{V}_{c h_{2}}=12500 \mathrm{rpm}$ (il faut un

\footnotetext{
15 "Resolution matching".
} 

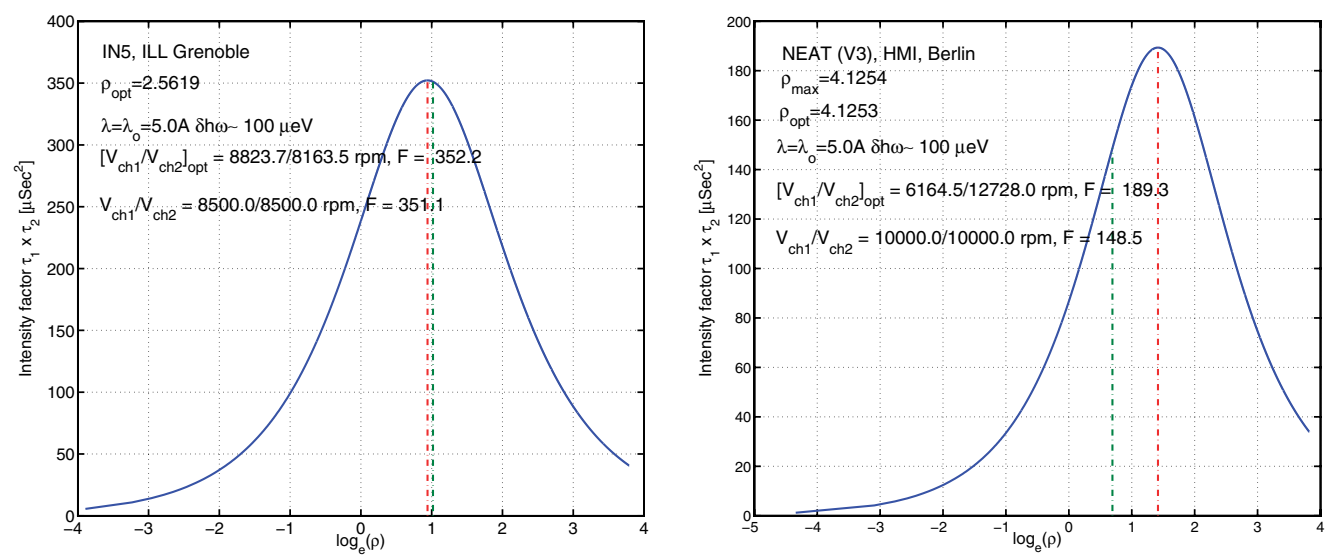

Figure 17. Facteur d'intensité $F=\tau_{1} \tau_{2}$ en fonction du ratio des largeurs de pulses pour une configuration standard, $5 \AA, \delta \hbar \omega \approx 100 \mu \mathrm{eV}$ pour IN5 et pour NEAT. Les lignes pointillées marquent la valeur théorique (maximum de la courbe correspondant à $\rho_{\text {opt }}$ ) (rouge) et la valeur réelle pour des valeurs de vitesses chopper égales : $\omega_{1}=\omega_{2}$ (vert). Les vitesses des choppers $\mathrm{V}_{c h_{i}}$ sont données pour le cas “idéal" correspondant au maximum d'intensité (donc à $\rho_{\text {opt }}$ ) et pour le cas réel.

rapport entier entre les vitesses). C'est cette configuration qui est optimisée pour $5 \AA$ avec un ratio $R=2$ (ou plus) pour les fentes de largeurs égales à la largeur du guide. Une autre possibilité est de choisir les fentes 2 fois plus larges pour la première paire de choppers. Dans ce cas $\alpha_{1} / \alpha_{2}=4.0$ et une configuration optimisée à vitesses de choppers égales est aussi possible ${ }^{16}$.

\subsubsection{Résolution spatiale}

Sur un temps de vol la résolution spatiale $\delta Q$ est généralement considérée comme petite devant les variations de Q. Dans la pratique, elle est donc souvent négligée dans l'analyse des données. Avec l'avènement des études sur monocristaux rendues possibles par l'usage des détecteurs PSD (Position Sensitive Detector), la connaissance de l'éllipsoide de résolution dans les quatre dimensions de l'espace réciproque devient impérative.

On peut distinguer deux cas : pour les études ne dépendant que du module de $\vec{Q}$, on peut calculer l'incertitude sur l'amplitude $|\vec{Q}|, \delta Q$ en partant de l'expression (3.26) et en suivant le raisonnement développé pour la résolution en énergie[30]. En plus de la dispersion en longueur d'onde due à la dispersion temporelle du faisceau incident (paragraphe 5.1.1), il faut tenir compte de la divergence $\delta \alpha$ du faisceau avant échantillon et de l'incertitude $\delta \phi$ sur l'angle de diffusion. On peut les ajouter quadratiquement dans le terme dépendant des angles.

En première approximation, pour une poudre ou un amorphe on obtient (exprimé en fonction des vitesses pour plus de clarté) :

$$
\delta Q=\frac{(2 \pi)^{2} C^{2}}{Q L_{12} L_{S D}}\left\{\begin{array}{l}
{\left[L_{S D} v_{i}^{3}-L_{2 S} v_{f}^{3}+v_{i} v_{f}\left(L_{2 S} v_{f}-L_{S D} v_{i}\right) \cos (\phi)\right]^{2} \delta t_{p}^{2}} \\
{\left[+\left(L_{12}+L_{2 S}\right) v_{f}^{3}-L_{S D} v_{i}^{3}-v_{f} v_{i}\left(\left(L_{12}+L_{2 S}\right) v_{f}-L_{S D} v_{i}\right) \cos (\phi)\right]^{2} \delta t_{m}^{2}} \\
{\left[+v_{f}^{2}\left(v_{i} \cos (\phi)-v_{f}\right)\right]^{4} \delta t_{d}^{2}+\left[v_{i} v_{f} \sin (\phi)\right]^{4}\left(\delta \phi^{2}+\delta \alpha^{2}\right)}
\end{array}\right\}^{\frac{1}{2}}
$$

\footnotetext{
16 Mais nécessite une vitesse plus élevée à résolution constante.
} 

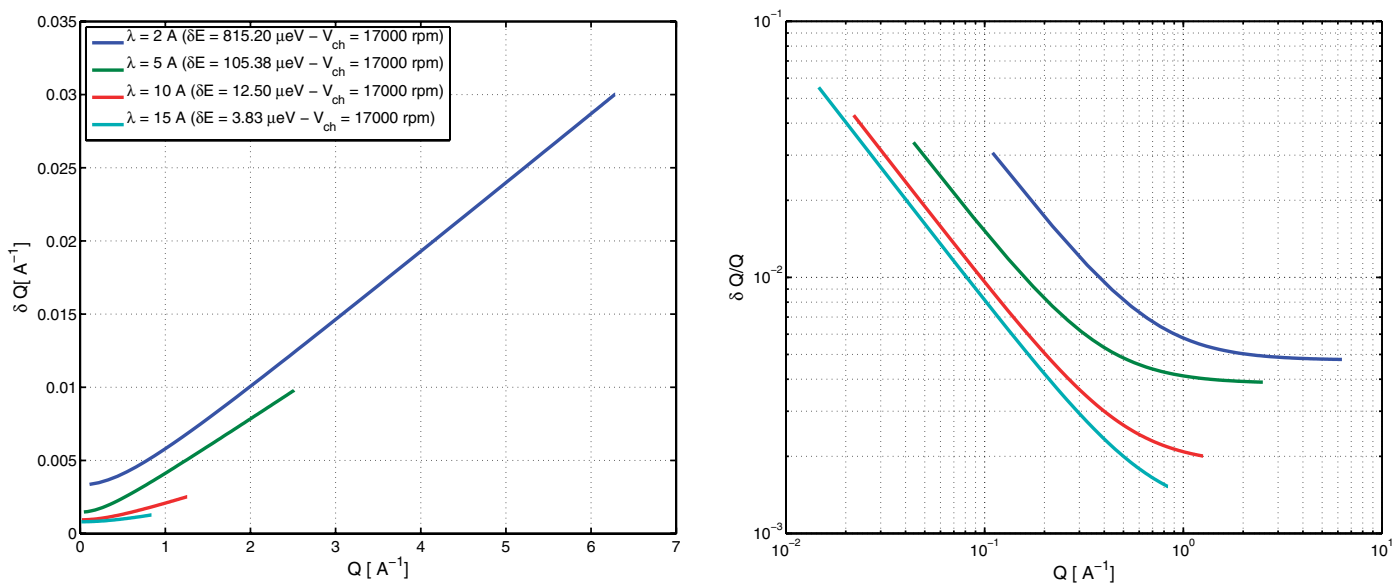

Figure 18. $\delta Q$ et $\delta Q / Q$ en fonction de $Q$ à transfert d'énergie nul dans l'approximation des équations (5.18) ou (5.19).

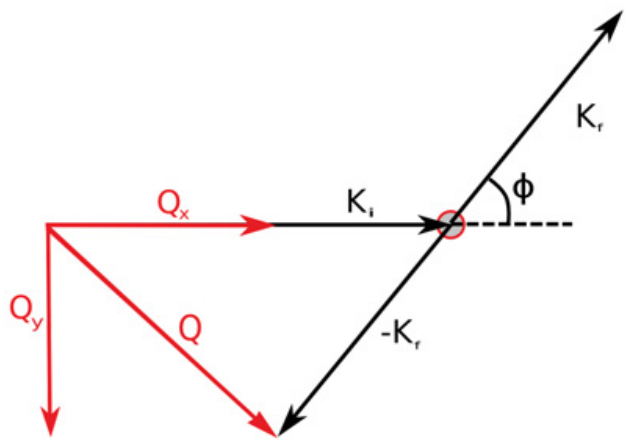

Figure 19. Définiton de $\delta Q_{\|}$et $\delta Q_{\perp}$ par rapport au vecteur d'onde incident $\vec{k}_{i}$ (dans la convention : $\vec{Q}=\vec{k}_{i}-\vec{k}_{f}$ et pour une diffusion sur des angles anti-horaires comme sur IN5).

ou, réduit à transfert d'énergie nul $\left(Q_{e}=2 \pi C v_{i} \sin \phi / 2\right)$ :

$$
\delta Q=\frac{Q_{e}}{2 L_{12} L_{S D}}\left[\begin{array}{l}
v i^{2}\left(\delta t_{d}^{2} L_{12}^{2}+\delta t_{m}^{2}\left(L_{12}+L_{2 S}-L_{S D}\right)^{2}+\delta t_{p}^{2}\left(L_{2 S}-L_{S D}\right)^{2}\right) \\
+\left(\delta \phi^{2}+\delta \alpha^{2}\right) L_{12}^{2} L_{S D}^{2} \cot ^{2}(\phi / 2)
\end{array}\right]^{\frac{1}{2}}
$$

Ici encore les paramètres $\delta t_{p}, \delta t_{m}$ et $\delta t_{d}$ s'identifient, respectivement, au temps d'ouverture des premiers choppers, de la dernière paire et à l'incertitude sur la distance de vol échantillon-détecteur (explicités au paragraphe 5.1.1).

La figure 18 montre l'évolution de la résolution en $\mathrm{Q}$ à transfert d'énergie nul pour quelques configurations du spectromètre IN5. Elle évolue proportionnellement à $\mathrm{Q}$ sauf aux petits angles où la divergence du faisceau domine (terme en $\delta \phi$ et $\delta \alpha$ ).

Pour les monocristaux, il est en principe nécessaire de connaître l'éllipsoïde de résolution dans toutes les directions de l'espace réciproque du cristal étudié. Mais on est jamais très fort pour ce calcul. En première approximation [30] on peut découpler les résolutions dans les directions parallèles $\delta Q_{\|}$et perpendiculaires $\delta Q_{\perp}$ au vecteur d'onde incident $\vec{k}_{i}$ (voir figure 19) : 

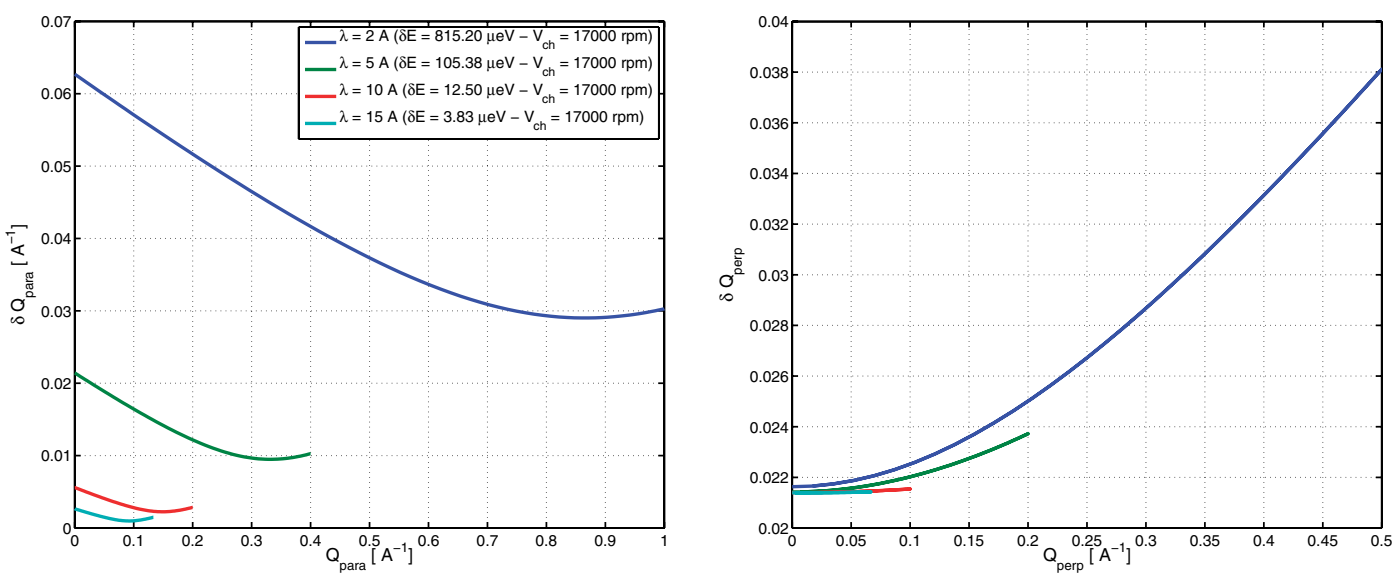

Figure 20. $\delta Q_{\|}$et $\delta Q_{\perp}$ en fonction de $Q_{\|}$et $Q_{\perp}$ à transfert d'énergie nul dans l'approximation de l'équation (5.21).

$$
\left\{\begin{array}{l}
Q_{\|}=k_{i}-k_{f} \cos \phi \\
Q_{\perp}=k_{i} \delta \alpha-k_{f} \sin \phi \\
Q_{z}=k_{i} \delta \beta_{i}-k_{f} \delta \beta_{f}
\end{array}\right.
$$

où l'on fait apparaître les divergences des faisceaux incidents et diffusés dans le plan $\left(\alpha_{i}\right)$ et perpendiculaire au plan de diffusion $\left(\beta_{i}, \beta_{f}\right)$.

$$
\left\{\begin{array}{l}
\delta Q_{\|}=\frac{2 \pi C}{L_{12} L_{S D}}\left[\begin{array}{l}
\left(L_{S D} v_{i}^{2}+L_{2 S} v_{f}^{2} \cos \phi\right)^{2} \delta t_{p}^{2} \\
+\left(L_{S D} v_{i}^{2}+\left(L_{12}+L_{2 S}\right) v_{f}^{2} \cos \phi\right)^{2} \delta t_{m}^{2} \\
+\left(L_{12} v_{f}^{2} \cos \phi\right)^{2} \delta t_{d}^{2} \\
+\left(L_{12} L_{S D} v_{f} \sin \phi\right)^{2} \delta \phi^{2}
\end{array}\right]^{\frac{1}{2}} \\
\delta Q_{\perp}=\frac{2 \pi C}{L_{12} L_{S D}}\left[\begin{array}{l}
\left(L_{2 S} v_{f}^{2} \sin \phi\right)^{2} \delta t_{p}^{2} \\
\left.+\left(L_{12}+L_{2 S}\right) v_{f}^{2} \sin \phi\right)^{2} \delta t_{m}^{2} \\
+\left(L_{12} v_{f}^{2} \cos \phi\right)^{2} \delta t_{d}^{2} \\
+\left(L_{12} L_{S D} v_{f} \cos \phi\right)^{2} \delta \phi^{2} \\
+\left(L_{12} L_{S D} v_{i}+\frac{\left(L_{12}+L_{2 S}\right)}{\omega} v_{f} \sin \phi\right)^{2} \delta \alpha^{2}
\end{array}\right]^{\frac{1}{2}} \\
\delta Q_{z}=\left[\delta \beta_{i}^{2} v_{i}^{2}+v_{f}^{2} \delta \beta_{f}^{2}\right]^{\frac{1}{2}}
\end{array}\right.
$$

Les résolutions dans le cas élastique sont montrées sur la figure 20 qui donne une idée de l'évolution de la forme de la section de la résolution dans le plan de diffusion.

En pratique, pour une meilleure prise en compte de la résolution dans l'espace à quatre dimensions pour les monocristaux il peut être nécessaire de se tourner vers une simulation de l'instrument incluant plus correctement, par exemple, les divergences avant et après échantillon ainsi que la géométrie de l'échantillon. Ceci est intégré, par exemple, dans des routines comme TOBYFIT à ISIS. On se rapproche alors de ce qui se fait couramment pour les spectromètres trois axes.

\subsection{Les spectromètres hybrides à géométrie directe du type IN6 (ILL)}

L'exemple canonique de ce type de spectromètres est IN6 à l'institut Laue-Langevin. La construction d'IN6 a été décidée en 1976 pour soulager la charge d'IN5 dans la gamme de neutrons froids $4 \AA$ - $6 \AA$. 

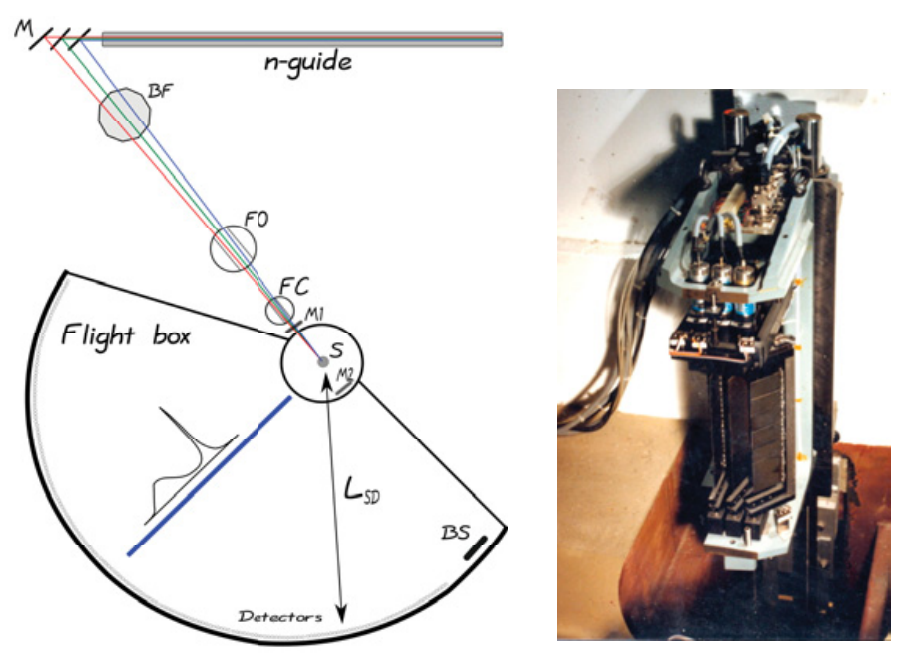

Figure 21. Schéma de principe du spectromètre IN6 à l'ILL, Grenoble. BF : filtre béryllium, FO : chopper (de type Fermi sans collimateur) d'antirecouvrement et bruit de fond, FC : chopper de Fermi, BS : beam stop. (droite) : Photo du triple monochromateur (M).

Ne pouvant pas disposer de bout de guide, il ne pouvait qu'utiliser un système de monochromateur(s) déviant une partie du faisceau par ailleurs utilisé en aval par d'autres instruments [29].

L'utilisation de plusieurs monochromateurs les uns à la suite des autres fut décidée pour augmenter le flux. Par contre, la disposition en file indienne des 3 monochromateurs (focalisants verticalement) ne leur permet pas d'avoir le même angle de diffraction afin de faire converger leur faisceau vers l'échantillon. Il est cependant possible de ne pas dégrader la résolution en utilisant les propriétés de focalisation en temps du spectromètre.

\subsubsection{Principe de la focalisation en temps}

Les faisceaux issus des différents monochromateurs présentent un décalage d'origine, mais un point de focalisation spatial commun sur l'échantillon (Fig. 22). La fonction première du chopper de Fermi, disposé entre les monochromateurs et l'échantillon, est de pulser le faisceau. Son ouverture angulaire importante permet, en plus, de balayer le faisceau issu du monochromateur de façon à transmettre les neutrons les plus lents (grande longueur d'onde) avant les plus rapides (petite longueur d'onde) pour que tous atteignent le détecteur en même temps (focalisation en temps). L'étalement temporel des instants de départ des neutrons d'un même pulse est :

$$
\Delta t=\frac{\phi}{\omega}=\frac{\phi}{2 \pi v}
$$

où $\phi$ est l'angle entre les faisceaux extrêmes vu de l'échantillon et $\omega$ la vitesse angulaire de rotation du chopper de Fermi.

La différence de longueur d'onde $\Delta \lambda$ entre les longueurs d'onde extrèmes est ( $\theta_{M}$ est l'angle de Bragg moyen pour la longueur d'onde nominale $\lambda_{i}$ ):

$$
\Delta \lambda=\lambda_{i} \cdot \phi / 2 \cdot \cot \theta_{M}
$$

Pour un transfert d'énergie nul, la différence de temps d'arrivée sur les détecteurs :

$$
\Delta \tau=C . L_{F D} \lambda \cot \theta_{M} \phi / 2
$$

avec $L_{F D}=L_{F S}+L_{S D}$ la distance Chopper de Fermi - détecteur. 

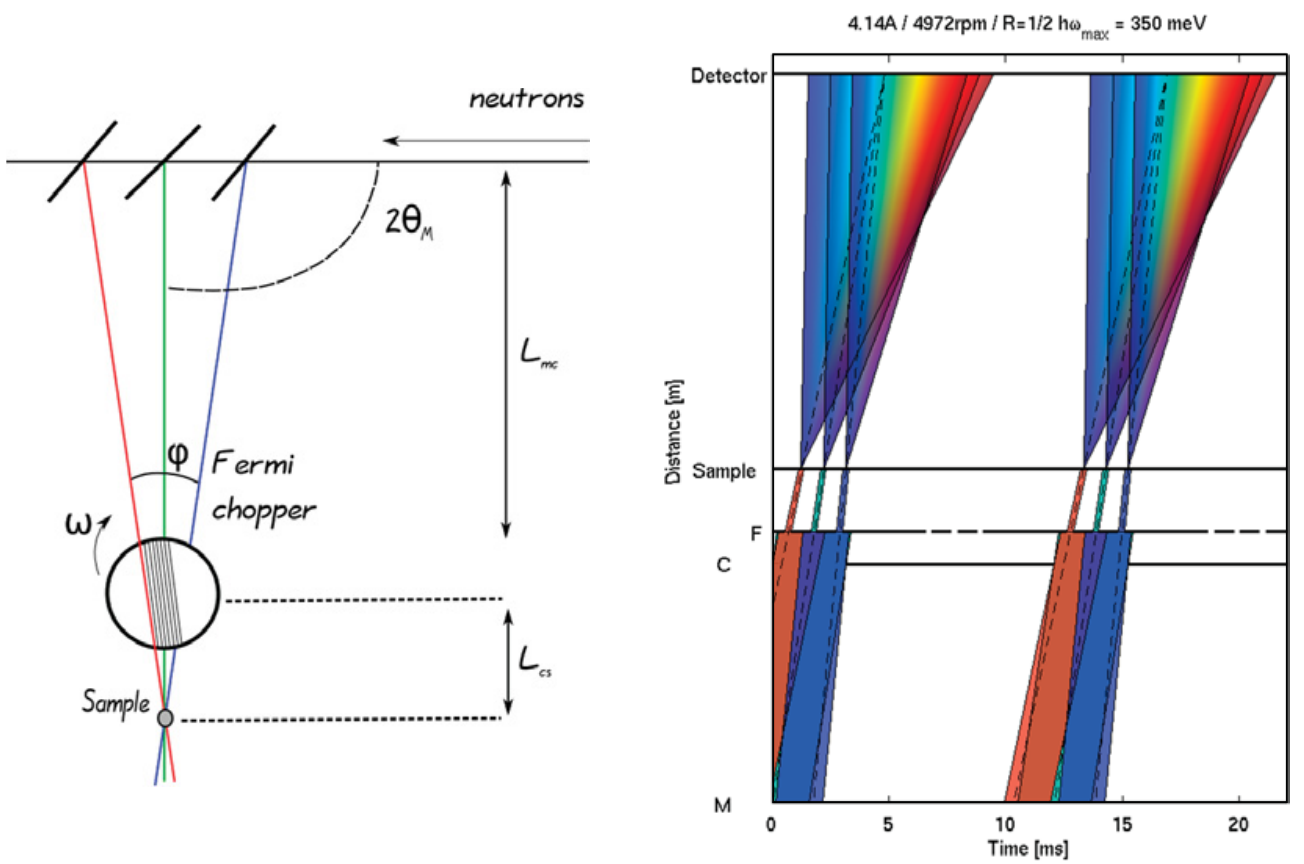

Figure 22. Gauche : Partie avant échantillon du spectromètre IN6. Droite : Diagrammes temps-distance pour une focalisation élastique (les distances entre monochromateurs ont été agrandies artificiellement pour mettre en évidence les 3 faisceaux).

La condition de focalisation revient à compenser le retard pris au départ $\Delta t$ par l'avance prise par les neutrons les plus rapides $\Delta \tau$ au point de focalisation, généralement au détecteur : $\Delta t=\Delta \tau$. Elle est remplie en ajustant la fréquence du chopper de Fermi à :

$$
v=\left[\pi C \lambda_{i}\left(L_{F S}+L_{S D}\right) \cot \theta_{M}\right]^{-1}
$$

Par construction, un instrument tel que IN6 sera donc toujours en focalisation en temps. Si il est naturel de focaliser sur l'énergie incidente afin d'avoir une résolution optimale pour la région quasiélastique, il est aussi possible de focaliser sur une région inélastique, de transfert d'énergie $E_{i}-E_{f}=\hbar \omega$, afin d'optimiser la résolution (principalement pour éviter la défocalisation) autour de modes ou de partie de densité d'états d'intéret.

Le temps de vol du paquet de neutron entre le Fermi et les détecteurs qui était de $T_{F D}=C \cdot L_{F D} \cdot \lambda_{i}$ devient, compte tenu que les neutrons diffusés sortent avec une longueur d'onde $\lambda_{f}$ après avoir échangé l'énergie $\hbar \omega$ :

$$
T_{F D}=C\left(L_{F S} \lambda_{i}+L_{S D} \lambda_{f}\right)
$$

La focalisation sur l'inélastique peut se faire en faisant varier les distances, par exemple $L_{S D}$, à fréquence du chopper de Fermi constante, ou plus simplement en faisant varier sa vitesse.

En différenciant l'expression 5.26 par rapport à $\lambda_{i}$ à transfert d'énergie $\hbar \omega$ constant et à l'aide de 5.5 :

$$
\delta T_{F D}=\left(L_{F S}+\left(\frac{\lambda_{f}}{\lambda_{i}}\right)^{3} L_{S D}\right) \delta \lambda_{i}
$$



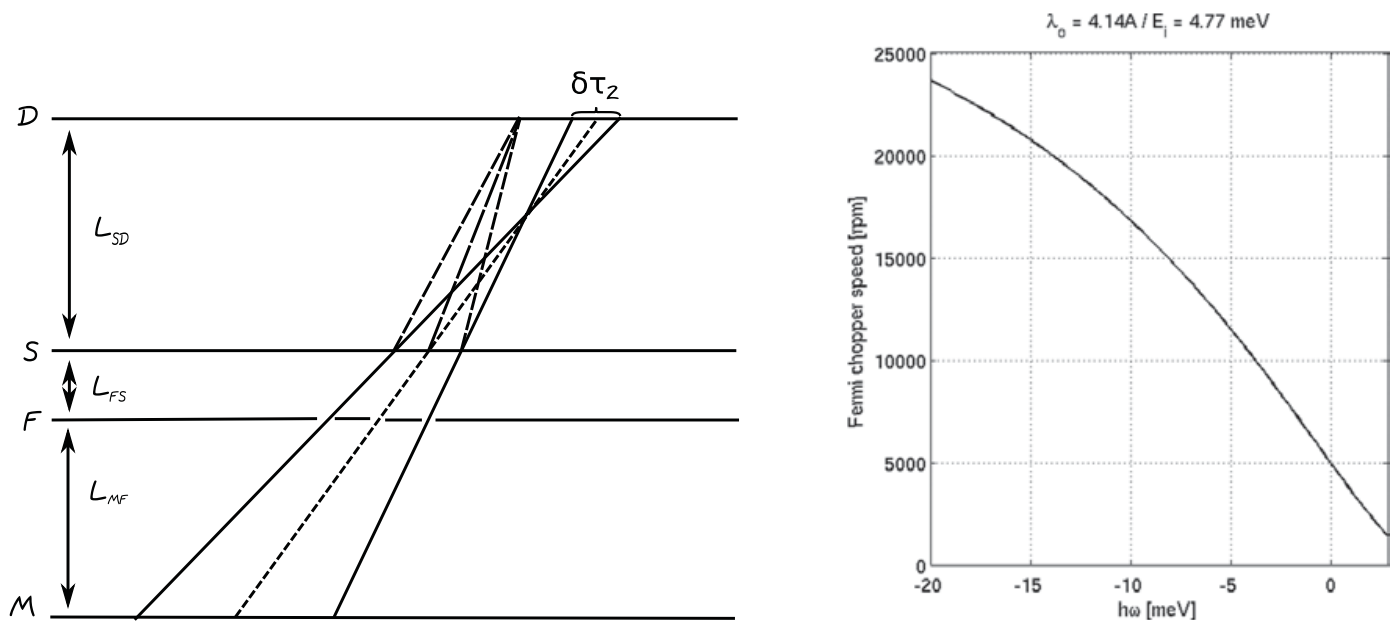

Figure 23. Gauche : Principe de focalisation en temps sur l'inélastique côté anti-Stokes (gain d'énergie pour le neutron). M : monochromateur, F : chopper de Fermi, S : échantillon, D : détecteur. Le diagramme montre les neutrons élastiques sans focalisation (ligne continue) et les neutrons focalisés à une énergie supérieure à l'énergie incidente (lignes pointillées). La focalisation sur l'inélastique du côté gain d'énergie pour les neutrons revient à focaliser les neutrons élastiques à une distance trop courte. Droite : Vitesse du chopper de Fermi en fonction du transfert d'énergie de focalisation.

La condition de focalisation en temps $\Delta t=\Delta \tau$ est remplie pour une fréquence du chopper de Fermi :

$$
v=\left[\pi C \lambda_{i} \cot \theta_{M}\left(L_{F S}+\left(\frac{\lambda_{f}}{\lambda_{i}}\right)^{3} L_{S D}\right)\right]^{-1}
$$

La figure 23 donne la dépendance de la vitesse du chopper de Fermi (en tours par minute - rpm) en fonction de la position en transfert d'énergie du point focal. Bien que la vitesse de rotation du chopper de Fermi augmente rapidement avec l'énergie de focalisation $\hbar \omega_{\text {foc }}$ elle reste dans des valeurs techniquement accessibles. La vitesse limite est compatible avec des dispositifs mécaniques usuels, d'après (5.28) :

$$
\lim _{\hbar \omega \rightarrow-\infty} v=\frac{\tan \theta_{M}}{\pi C \lambda_{i} L_{F S}}
$$

Comme on l'a vu dans la section 3.3.1 la résolution en énergie est donnée par l'étalement, au niveau du détecteur, des temps d'arrivées des neutrons ayant subi un même transfert d'énergie $\hbar \omega$ (Eq. (3.18)). C'est une spécificité de la focalisation en temps, de pouvoir adapter la meilleure résolution à la gamme de transfert d'énergie que l'ont désire sonder.

Il y a tout de même un prix à payer sur l'intensité puisque l'augmentation de la vitesse du chopper de Fermi produit des paquets de neutrons plus courts donc moins intenses. Il devient, de plus, nécessaire d'augmenter le ratio $\mathrm{R}$ afin d'éviter une situation de recouvrement des pulses successifs au niveau des détecteurs (overlap). En plus d'éviter la défocalisation pour un transfert d'énergie non-nul, une amélioration de la résolution est donc aussi obtenue par l'accroissement de la vitesse du chopper de Fermi $^{17}$.

${ }^{17}$ Ceci réduit l'intérêt de la technique au côté Anti-Stokes de l'échantillon puisque du côté Stokes il faut ralentir le Fermi. 


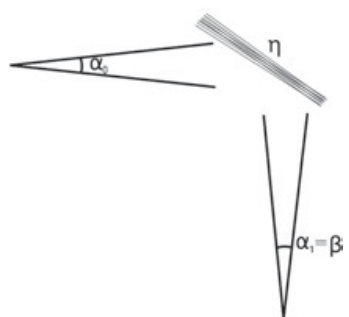

Figure 24. Schéma de principe pour la dispersion $\delta \lambda$ d'un monochromateur de mosaïcite $\eta$ voyant un faisceau de divergence incidente $\alpha_{0}$ et diffusée $\alpha_{1}$.

\subsubsection{Fonction de résolution}

Le calcul de la résolution en énergie d'un spectromètre hybride est un peu plus compliquée que pour l'instrument à choppers. Comme au paragraphe précédent, on s'accorde à considérer des contributions indépendantes et de forme gaussienne pour utiliser les propriétés magiques de sommation en quadrature des largeurs lors de la convolution.

A partir de :

$$
\frac{\delta \hbar \omega}{E_{f}}=2 \frac{\delta t}{t}
$$

où $t=C L_{S D} \lambda_{f}$ est le temps de vol entre l'échantillon et le détecteur et $\delta t$ son incertitude totale, on a :

$$
\delta t^{2}=\sum_{i} \delta t_{i}^{2}
$$

Nous discutons ci-après de l'expression la plus simple donnée par R. Scherm et co-auteurs [29] lors de la conception de IN6. Si elle n'est pas la plus complète elle permet de mettre clairement en évidence les termes en jeu dans la résolution.

La décomposition de $\delta t$ se fait en quatre parties :

\section{Monochromateur et chopper de Fermi}

La dispersion de la longueur d'onde incidente $\delta \lambda_{i}$ de chaque monochromateur de mosaïcité $\eta$ sous un faisceau sortant du guide avec la divergence $\alpha_{0}$, et vu sous l'angle de collection $\alpha_{1}$ à l'échantillon, est de [31] :

$$
\delta \lambda_{i}=\lambda_{i} \cot \theta_{M} \delta \theta
$$

avec (voir Fig. 24) :

$$
\delta \theta=\left(\frac{\alpha_{\circ}^{2} \eta^{2}+\alpha_{1}^{2} \eta^{2}+\alpha_{\circ}^{2} \alpha_{1}^{2}}{\alpha_{\circ}^{2}+\alpha_{1}^{2}+4 \eta^{2}}\right)^{\frac{1}{2}}
$$

$\alpha_{\circ}$ est généralement la divergence naturelle du guide, $\alpha_{\circ}[\AA] \simeq 0.1 N \lambda[\AA ̊](\mathrm{N}$ index du super-mirroir, 1 si nickel naturel) et $\alpha_{1}$ se confond avec la collimation $\beta$ du chopper de Fermi. Pour un monochromateur de grande mosaïcité (l'intensité croit avec la mosaïque et celle-ci n'agit pas sur la résolution) $\eta \gg \alpha_{\circ}, \beta$ l'expression 5.33 se réduit à ;

$$
\delta \theta \simeq \frac{\sqrt{\alpha_{\circ}^{2}+\beta^{2}}}{2}
$$


En réutilisant l'équation (5.27) et en combinant avec Eq. (5.32) et (5.36) on obtient la dispersion en temps au détecteur au travers du système monochromateur et chopper de Fermi ${ }^{18}$ :

$$
\delta t_{1}=C \frac{\lambda_{i}}{2} \sqrt{\alpha_{\circ}^{2}+\beta^{2}} \cot \theta_{M}\left(L_{F S}+\left(\frac{\lambda_{f}}{\lambda_{i}}\right)^{3} L_{S D}\right)
$$

\section{Défocalisation}

Par principe la focalisation n'intervient que pour l'énergie de focalisation $\hbar \omega_{\mathrm{foc}}$ au temps de focalisation $t_{\text {foc }}$. Partout ailleurs les 3 faisceaux se défocalisent en fonction de leur éloignement à l'énergie de focalisation (figure 23).

À la section 5.2.1 on a vu que la condition de focalisation était réalisée pour $\Delta t=\Delta \tau$. Mais hors focalisation, $\Delta t \neq \Delta \tau$ ce qui dégrade la résolution de $\delta t_{2}=\Delta t-\Delta \tau$, soit :

$$
\delta t_{2}=\phi\left[C \frac{\lambda_{i}}{2} \cot \theta_{M}\left(L_{F S}+\left(\frac{\lambda_{f}}{\lambda_{i}}\right)^{3} L_{S D}\right)-\frac{1}{2 \pi \nu}\right]
$$

\section{Échantillon :}

La base de temps de vol étant courte sur les instruments hybrides sur neutrons froids, l'incertitude sur la distance échantillon - détecteur va être relativement importante. Le calcul est similaire à celui du paragraphe (5.1.1).

\section{Détecteurs}

Pour les mêmes raisons, l'incertitude sur la localisation de la détection dans le détecteur est nonnégligeable. Comme précédemment, la probabilité de détection est considérée homogène dans le détecteur quelque soit la longueur d'onde, on considère l'incertitude sur le temps de vol comme inversement proportionelle à la vitesse des neutrons diffusés (voir section 5.1.1).

La résolution en énergie est calculée à partir de (5.30) et (5.31) sur la figure (25) pour un temps de vol fictif mettant en valeur les termes discutés plus haut. La résolution déduite de IN6 dans sa configuration actuelle est mise pour comparaison.

La défocalisation est très dépendante de l'angle d'acceptance $\phi$. Minimiser la distance, dans la limite de ce qui est possible mécaniquement, entre les monochromateurs, permet alors de diminuer sensiblement ce terme (voir la photo sur la figure 21).

\subsubsection{Conclusions}

Un spectromètre comme IN6 est très "rentable". En n'étant pas sur une fin de guide il ne prélève que les neutrons en condition de Bragg sur ses monochromateurs laissant le reste du faisceau "irriguer" d'autres instruments en aval. La totalité des autres instruments à temps de vol perd les neutrons qui ne sont pas utilisés.

Les distances en jeux dans sa géométrie ne permettent pas, en revanche, d'éliminer les harmoniques du monochromateurs $(\lambda / 2, \lambda / 3, \ldots)$ qui, même peu intenses, sont une source de pollution pour un temps de vol. Pour éviter cette contamination, IN6 utilise donc un filtre béryllium refroidi qui a la particularité de ne transmettre que les longueurs d'ondes $\lambda \geq 3.95 \AA$. La gamme de longueur d'onde incidente accessible se limite alors à : $4.0 \AA \leq \lambda<6.69 \AA\left(\lambda_{002}=2 d_{002}\right)$, réalisable avec un monochromateur de graphite. Pour des raisons d'encombrement de l'instrument il est même limité à $6.0 \AA ̊$.

\footnotetext{
${ }^{18}$ Un calcul complet[32] permet de prendre en compte le couplage existant entre les différents paramètres considérés ici comme indépendants.
} 

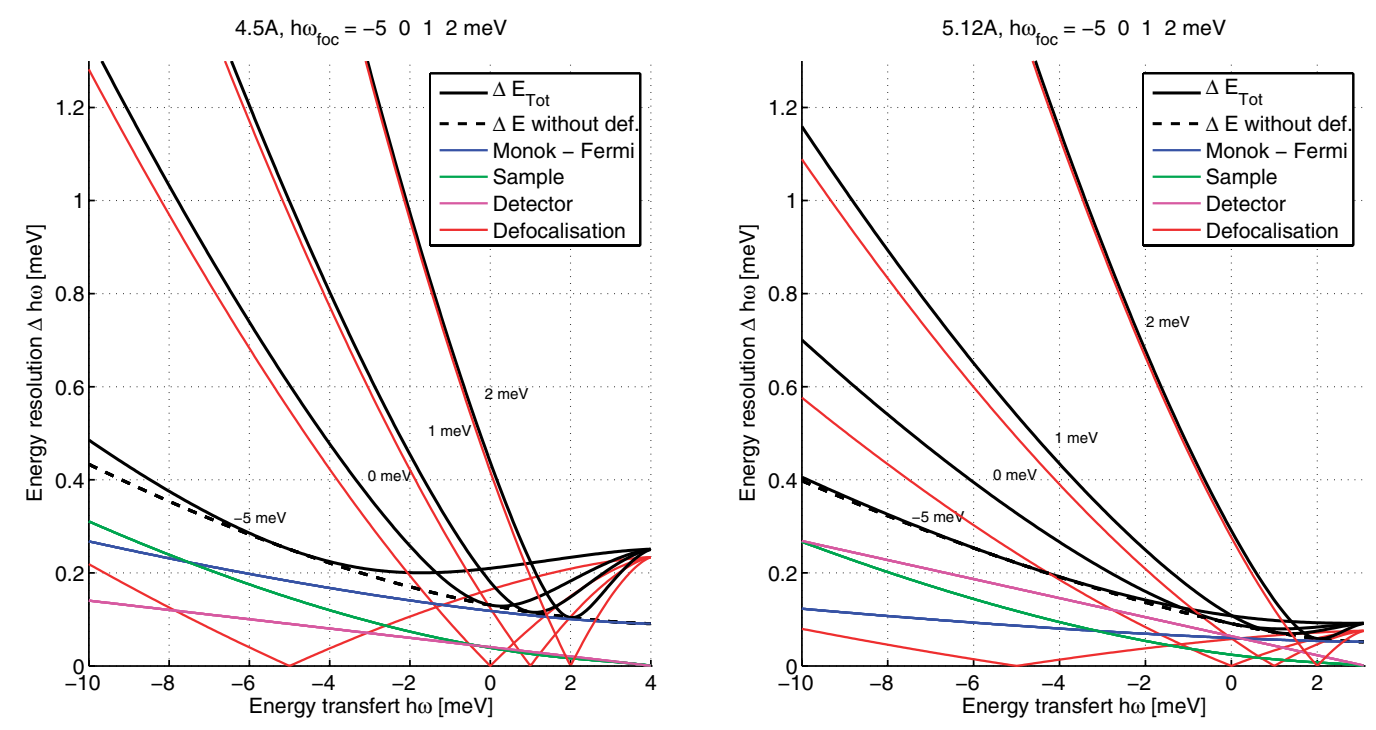

Figure 25. (Gauche) Résolution d'un spectromètre du type d'IN6 pour des paramètres fictifs : $L_{F S}=0.6 \mathrm{~m}$, $L_{S D}=2.0 \mathrm{~m}, \alpha_{\circ}=0.6^{\circ}, \beta=1.0^{\circ}, \phi=3.0^{\circ}, \Phi=90^{\circ}, \psi=45^{\circ}, w=3.0 \mathrm{~cm}, e=1.0 \mathrm{~cm}, D=1.0 \mathrm{~cm}$. (Droite) pour IN6 pour différentes conditions de focalisation: $L_{F S}=0.385 \mathrm{~m}, L_{S D}=2.48 \mathrm{~m}, \alpha_{\circ}=0.1 \times \lambda_{i}\left[^{\circ}\right]$, $\beta=1.0^{\circ}, \phi=1.6^{\circ}, \Phi=90^{\circ}, \psi=45^{\circ}, w=3.0 \mathrm{~cm}, e=1.0 \mathrm{~cm}, D=2.54 \mathrm{~cm}$.

Tous les instruments n'ont pas ces limitations. Un instrument comme IN4 à l'ILL est équipé d'un système de 2 choppers de présélection de la bande d'énergie qui permet d'éliminer les harmoniques du monochromateur ou, au contraire, de les utiliser comme longueur d'onde incidente.

Un instrument comme FOCUS à PSI s'affranchir des harmoniques en plaçant à la bonne distance les choppers de Fermi et de background/antirecouvrement. Cela lui permet, entre autre, d'utiliser alternativement un monochromateur Mica à la place du graphite pour avoir accès aux grandes longueurs d'onde dont les harmoniques ne seraient pas coupées par un filtre béryllium $\left(2 d_{002}=19.9 \AA\right)$.

\subsection{Les spectromètres hybrides à géométrie directe du type FOCUS (PSI)}

Le spectromètre FOCUS au Paul Scherrer Institut de Willigen est une version aboutie du temps de vol hybride direct car étant sur une fin de guide et n'ayant donc pas les contraintes de construction qu'avait IN6 à son époque [33,34].

Le chopper d'anti-recouvrement de spectre est placé juste après la fin du guide qui peut être considéré comme une source ponctuelle.

Un grand monochromateur $M$ doublement focalisant est placé à une distance $L_{G M}$ du guide $G$, il focalise géométriquement le faisceau sur l'échantillon $S$ placé à une distance $L_{M S}$ du monochromateur.

\subsubsection{Mode focalisation monochromatique (MF)}

Contrairement à IN6, il peut fonctionner sans la focalisation en temps en se plaçant dans la géométrie de Johansson [35] (Fig. 26). Dans ce cas, quand $L_{G M}=L_{M S}$ tous les monocristaux constituant le monochromateur ont le même angle de Bragg $\theta_{M}$ et c'est un faisceau large mais monochromatique, qui converge vers l'échantillon.

Limiter l'angle d'acceptance du chopper Fermi est alors inutile (on peut enlever la collimation du Fermi), la meilleure résolution sera obtenue en faisant tourner le plus vite possible le chopper de Fermi 

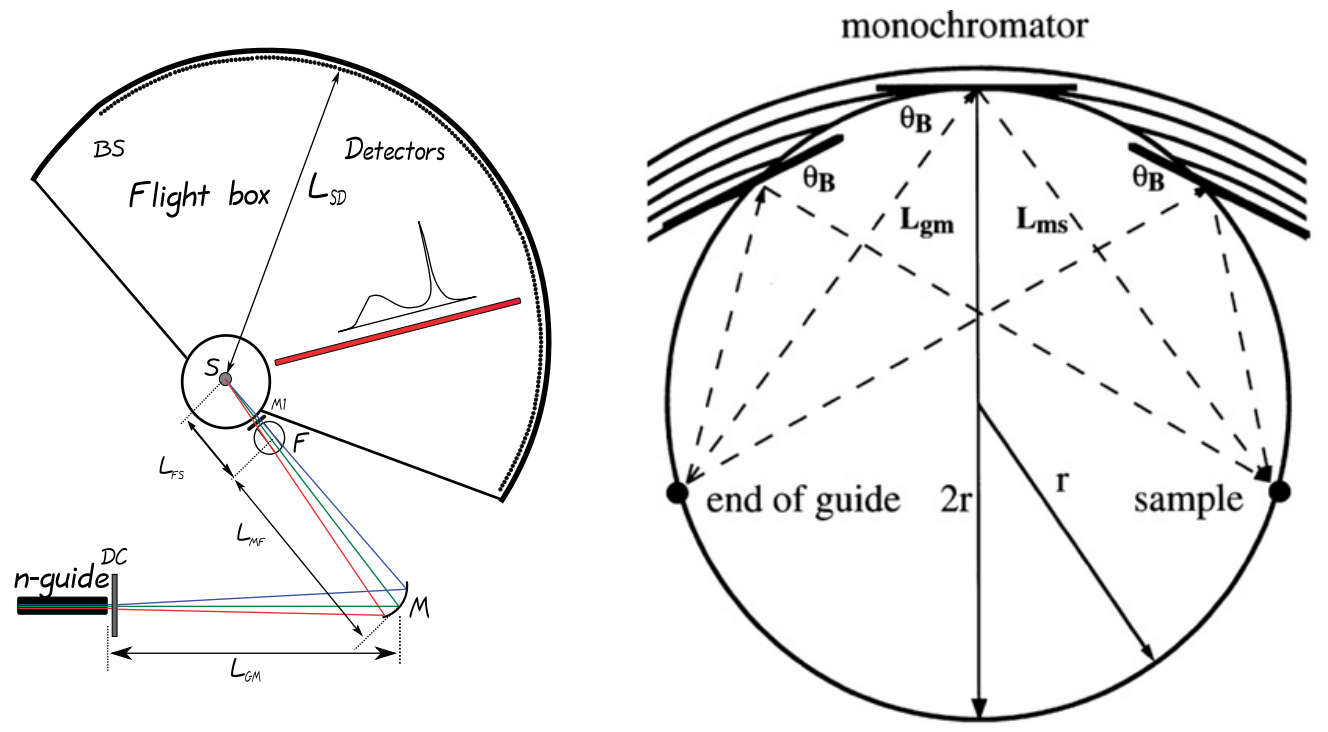

Figure 26. Gauche : Schéma de principe du spectromètre FOCUS à PSI-SINQ. Droite : Cercle de Rowland de géométrie focalisante [37]. La focalisation monochromatique est obtenue si $L_{G M}=L_{M S}$.
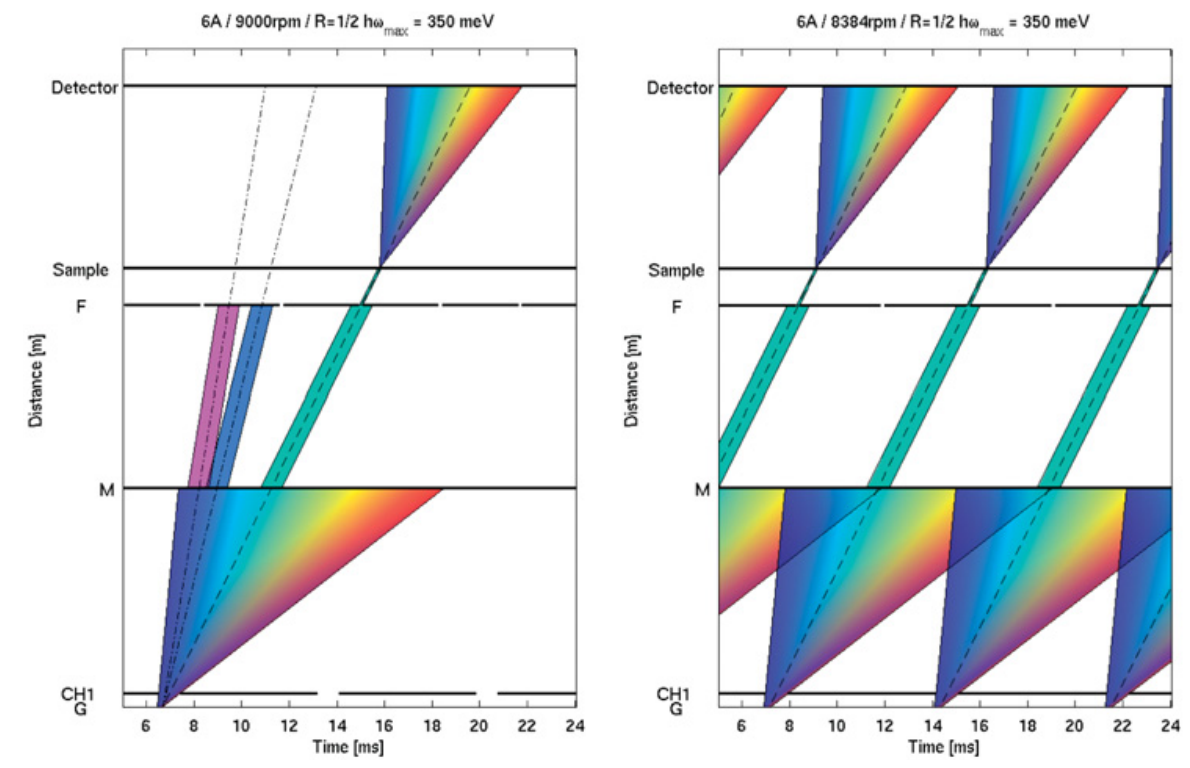

Figure 27. Gauche : Diagramme temps-distance pour FOCUS en mode de focalisation monochromatique (MF). L'élimination des harmoniques $(\lambda / 2$ et $\lambda / 3)$ par un choix judicieux des phases des choppers est aussi montré. Droite : Idem pour une configuration réelle (les distances sont à l'échelle). G : fin du guide, CH1 : chopper de background, $\mathrm{M}$ : monochromateur, F : chopper de Fermi.

dans la limite du flux obtenu. L'instrument possède 2 configurations en MF :

- Mode basse résolution / haut flux : $L_{G M}=L_{M S}=1.5 \mathrm{~m}$ en plaçant un guide entre le chopper d'anti-recouvrement et le monochromateur et en faisant varier la distance du spectromètre secondaire monté sur coussin d'air.

- Mode haute résolution / flux modéré : $L_{G M}=L_{M S}=3.0 \mathrm{~m}$ en enlevant le guide intermédiaire. 


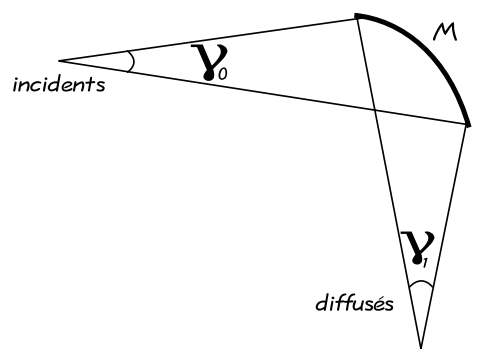

Figure 28. Schéma de la dispersion d'un monochromateur focalisant.

\subsubsection{Mode focalisation temporelle (TF)}

La flexibilité sur les distances permet de se mettre en géométrie focalisante en temps. Le plus avantageux étant de se placer à $L_{G M}<L_{M S}$, c.a.d. $L_{G M}=1.5 \mathrm{~m}$ et $L_{M S}=3.0 \mathrm{~m}$, pour focaliser sur l'inélastique côté gain d'énergie pour le neutron. Ceci est possible en intercalant un guide après le premier chopper permettant de reporter le "point source", c.a.d, la fin du guide, plus en aval.

La bande en longueur d'onde $\delta \lambda_{i}$ délivrée par un monochromateur courbe focalisant un point source sur un point objet peut être approximée, dans le cas ou $\gamma_{\circ} \neq \gamma_{1}$, par [32] (voir figure 28) :

$$
\delta \lambda_{i}=\frac{\lambda_{i}}{2} \cot \theta_{M}\left|\gamma_{0}-\gamma_{1}\right|
$$

Par symétrie de la réflexion sur le monochromateur, on aura toujours :

$$
\frac{\gamma_{0}}{\gamma_{1}}=\frac{L_{G M}}{L_{M S}}
$$

En combinant les Eqs. (5.37), (5.27) et (5.38) on obtient la vitesse du chopper de Fermi pour avoir le focus pour le transfert d'énergie $\hbar \omega$ :

$$
v=\left[\pi C \lambda_{i} \cot \theta_{M}\left(1-\frac{L_{M S}}{L_{G M}}\right)\left(L_{F S}+\left(\frac{\lambda_{f}}{\lambda_{i}}\right)^{3} L_{S D}\right)\right]^{-1}
$$

Sur le schéma Fig. 28 aligner les monochromateurs comme sur IN6 revient à la situation $\gamma_{0}=0$ soit $L_{G M} \rightarrow \infty$.

Le spectromètre sur neutrons thermiques IN4 à l'ILL se trouve dans cette situation avec le point source virtuel (équivalent de la fin du guide sur FOCUS) remplacé par un diaphragme et tel que $L_{M S}<L_{G M}$ ( $L_{G M}$ et $L_{M S}$ variables en fonction de la longueur d'onde incidente). Il fonctionne donc par construction en mode TF avec la vitesse du Fermi asservie à l'énergie de focalisation.

\subsubsection{Fonction de résolution}

Les fonctions de résolution se déduisent de celles vues pour IN6, sans terme de défocalisation $\delta t_{2}=0$ pour le mode MF mais avec une acceptance $\beta$ plus grande pour le chopper de Fermi.

En mode de focalisation temporelle (TF) le terme $\delta \lambda_{i}$ est changé selon (5.37) et (5.38). Le terme de défocalisation devient :

$$
\delta t_{2}=\phi\left[C \frac{\lambda_{i}}{2} \cot \theta_{M}\left(1-\frac{L_{G M}}{L_{M S}}\right)\left(L_{F S}+\left(\frac{\lambda_{f}}{\lambda_{i}}\right)^{3} L_{S D}\right)-\frac{1}{2 \pi v}\right]
$$

La figure 29 montre la résolution en mode MF et TF pour FOCUS. Attention : les configurations correspondantes à ces résolutions ne sont pas équivalentes en terme de flux. 

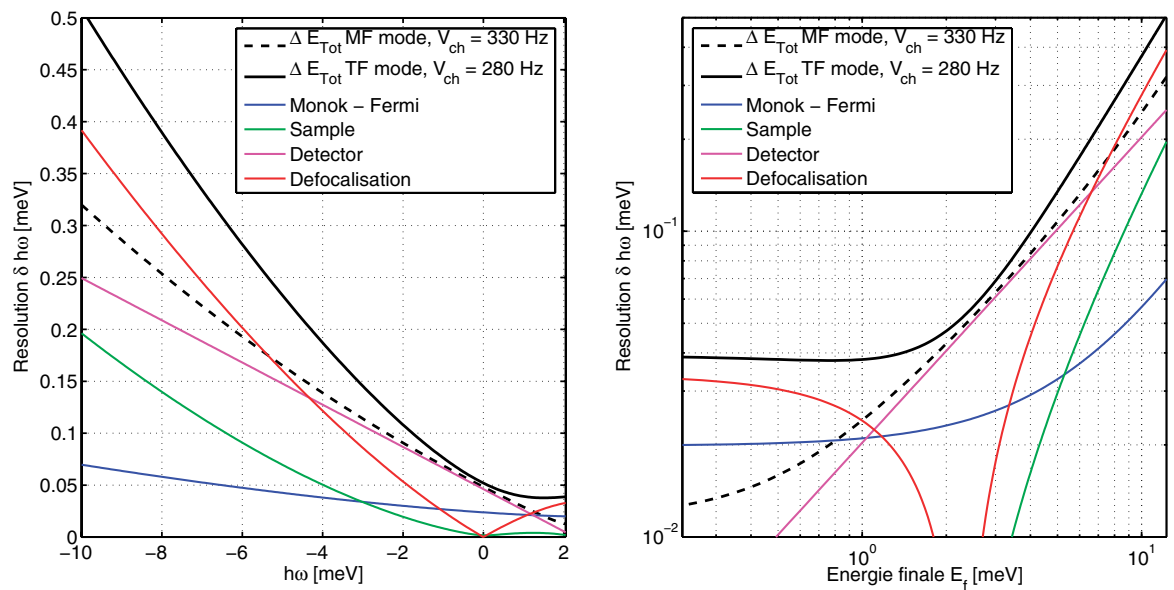

Figure 29. Gauche : Résolutions en fonction de l'énergie pour le spectromètre FOCUS, PSI-Willigen pour les modes TF et MF. Droite : Idem en échelle logarithmique en fonction de l'énergie finale.

\subsection{Les spectromètres hybrides à géométrie inverse}

Ils se retrouvent surtout sur sources à spallation bien qu'il ne soit pas impossible de les construire sur réacteur. Au lieu de filtrer la longueur d'onde incidente avant l'échantillon c'est un pulse polychromatique (éventuellement directement le pulse du modérateur) qui est amené sur l'échantillon après s'être dispersé le long de la (longue) base de vol du spectromètre primaire $L_{M S}$.

L'analyse se fait après l'échantillon, à la manière d'un spectromètre à rétrodiffusion ou de l'analyseur d'un trois-axes, en filtrant les neutrons d'énergie fixée par un analyseur. Le spectre est enregistré en temps, l'intensité de chaque canal étant normalisé au flux incident (moniteur avant échantillon) car l'intensité du spectre incident varie selon une forme caractéristique de la source.

Un avantage de ce type d'instrument sur les appareils à géométrie directe est qu'il utilise une gamme d'énergies incidentes étendue et très supérieure à l'énergie diffusée par l'analyseur. Le spectre final est donc essentiellement enregistré du côté perte d'énergie du neutron, donc du côté Stokes pour l'échantillon, à l'opposé des specromètres à géométrie directes qui travaillent surtout en gain d'énergie pour le neutron et voient le côté Stokes ne pas excéder, au mieux, l'énergie des neutrons monochromatiques incidents. Cependant, en pratique la gamme de transfert d'énergie est tout de même limitée par la répétition des pulses (Fig. 31) et par le type de neutrons utilisés (thermiques ou froids).

Connaissant précisément les distances source (ou modérateur) - échantillon $L_{M S}$, échantillon détecteur $L_{S D}=L_{S A}+L_{A D}$, et le temps total $t=t_{\mathrm{det}}-t_{\circ}$ écoulé depuis la génération à $t_{\circ}$ du pulse initial jusqu'au détecteur, l'enregistrement en temps du signal après analyseur permet de mesurer l'énergie échangée au niveau de l'échantillon :

$$
\hbar \omega=\frac{1}{2} m_{n}\left[\left(\frac{L_{M S}}{t-t_{S D}}\right)^{2}-\left(\frac{L_{S D}}{t_{S D}}\right)^{2}\right]
$$

\subsubsection{Fonction de résolution}

Comme pour les autres spectromètres Eq. (3.18) :

$$
\frac{\delta E}{E_{f}}=2 \frac{\delta \lambda}{\lambda}=2 \frac{\delta t}{t_{M D}}
$$




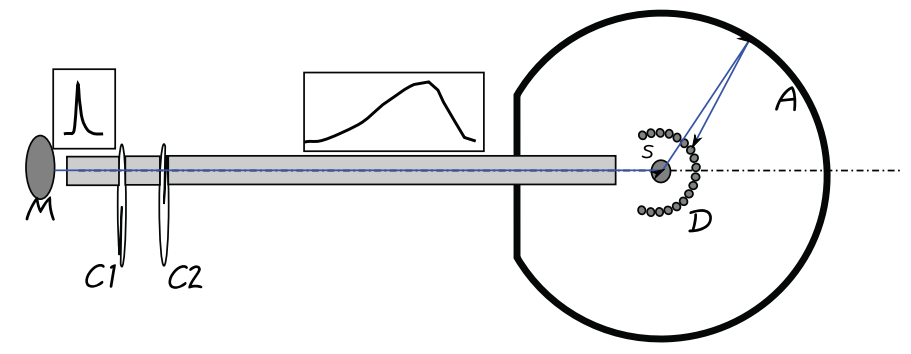

Figure 30. Schéma d'un spectromètre hybride à géométrie inverse en quasi-rétrodiffusion tel que IRIS à ISIS. M : Modérateur (source), C1, C2 : choppers de mise en forme et pré-monochromatisation (C2 fait aussi antirecouvrement) A : Analyseurs, D : détecteurs.

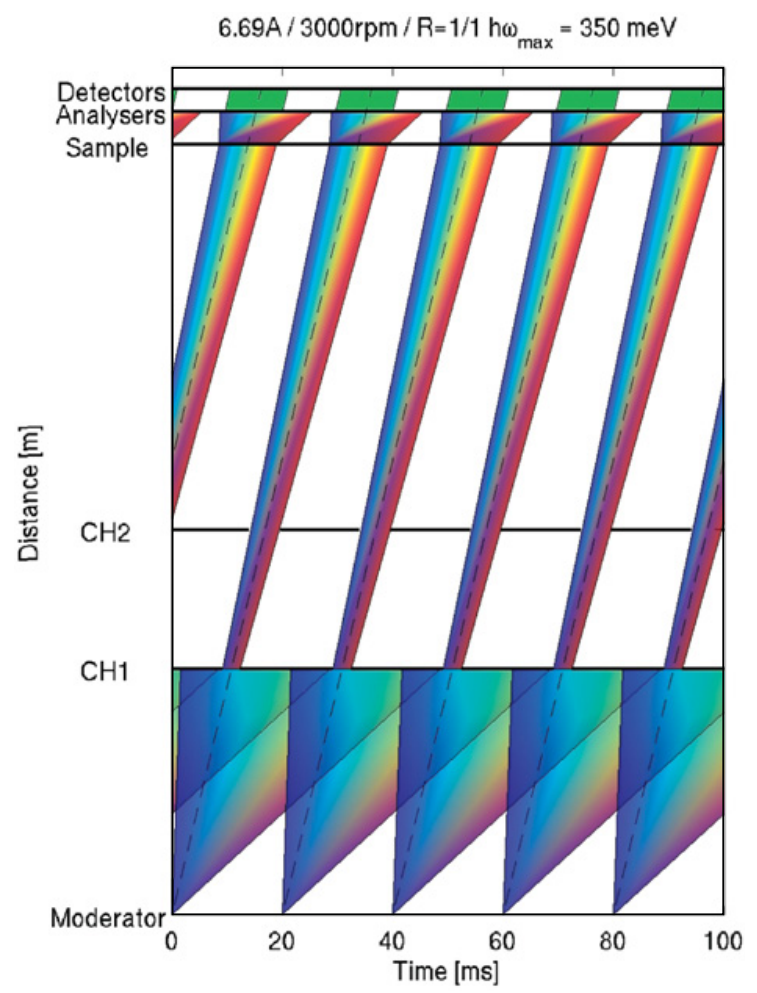

Figure 31. Diagramme temps-distance pour un spectromètre tel que IRIS (Attention : les dimensions ne sont pas respectées). A : Analyseurs, D : détecteurs.

où $t_{M D}$ est le temps de vol source (modérateur) - détecteur. Le terme $\delta t / t_{M D}$ dépend des élargissements induits par les étalements temporels du pulse à la source, $\delta t_{\text {Mod, }}$ et au niveau du spectromètre secondaire $\delta t_{S D}$ (échantillon - détecteur) :

$$
\frac{\delta t_{S D}}{t_{S D}}=\frac{\delta \lambda_{A}}{\lambda_{A}}=\left[\left(\frac{\delta d_{h k l}}{d_{h k l}}\right)^{2}+\left(\cot \theta_{A} \delta \theta_{A}\right)^{2}\right]^{\frac{1}{2}}
$$

obtenu en différentiant la loi de Bragg (terme commun aux spectromètres à rétrodiffusion) où $t_{S D}=$ $C L_{S D} \lambda_{002}$ est le temps de vol entre le modérateur et les détecteurs pour la longueur d'onde $\lambda_{002}=$ 
$6.69 \AA$ réfléchie en rétrodiffusion par le graphite, $\delta d_{h k l} / d_{h k l}$ est l'incertitude sur l'espacement des plans réticulaires des analyseurs et le dernier terme l'incertitude sur la longueur d'onde dispersée par l'analyseur à l'angle de Bragg $\theta_{A}$.

\subsubsection{Optimisation}

Ces instruments présentent toujours un spectromètre primaire très long, de l'ordre de 30 à 40 m, de façon à optimiser la résolution [38]. On admet qu'un optimum de résolution est obtenu lorsque l'incertitude due au spectromètre primaire $\frac{\delta t_{\text {Mod }}}{t_{M D}}$ est égale à l'incertitude due au spectromètre secondaire ${ }^{19}$. Soit, en parfaite rétrodiffusion (le $3^{\text {ème }}$ terme est négligeable) $\frac{\delta t_{\text {Mod }}}{t_{M D}} \approx \frac{\delta d_{h k l}}{d_{h k l}}$. Dans ce cas, la durée d'un pulse modérateur sur IRIS étant de la forme $\delta t_{\operatorname{Mod}}[\mu s] \approx 22 \lambda[\AA]$, et $\delta d_{h k l} / d_{h k l} \simeq 210^{-3}-2.510^{-3}$ pour le graphite, la longueur totale $L_{M D}=L_{M S}+L_{S D}$ est fixée par construction à $L_{M D}=22 / C .210^{-3} \simeq$ 34.8 - $43.5 \mathrm{~m}$ (valeur retenue : $38 \mathrm{~m}$ ).

Cette distance est assez grande et n'est pas forcément compressible. A titre d'exemple, le calcul d'optimisation de la distance premier chopper - détecteur du spectromètre MARS donne $41.8 \mathrm{~m}$ [39].

\section{CONCLUSION}

Cet article a précisé les enjeux et les contraintes techniques rencontrées sur les différents types de spectromètres de neutrons à temps de vol. La solution la plus naturelle pour parvenir à une résolution en énergie entre 10 et $100 \mu \mathrm{eV}$, utilisable lors de l'étude de la dynamique de la matière condensée, est de limiter à quelques pourcents l'incertitude en énergie des neutrons incidents. C'est la stratégie adoptée dans la conception des spectromètres à choppers (Mibémol, IN5, NEAT, TOFTOF, DCS...). Ces machines sont très versatiles : le changement de la longueur d'onde incidente est simplement commandé par le choix des phases entre les choppers, si bien qu'une modification de configuration (longueur d'onde, gamme en Q, résolution...) ne prend que quelques minutes. La courbe de résolution, de forme gaussienne "sans ailes", permet en outre de détecter des signaux quasi-élastiques très peu intenses et/ou plus étroits que la résolution. Le coût des ces avantages est une luminosité relativement faible.

Une alternative à la solution précédente permet d'obtenir des résolutions dans la même gamme, mais avec un flux d'un ordre de grandeur supérieur. L'astuce consiste à élargir significativement la gamme de longueurs d'onde des neutrons incidents, mais retarder l'instant de départ des neutrons les plus rapides pour qu'ils ne rattrapent les plus lents qu'au niveau des détecteurs. Ce décalage des temps de départ permet de maintenir une incertitude sur le temps de vol relativement faible mais dans une gamme limitée d'énergie. C'est le principe de focalisation en temps, mis en oeuvre sur IN6 à l'ILL. Il rend possible l'utilisation d'une bande relativement large du spectre "blanc" (distribution approximativement Maxwellienne) de neutrons acheminés par le guide. Le gain d'intensité important par rapport à la technologie à choppers découle en partie de ce point. La focalisation en temps est réalisée par un ou plusieurs monochromateur(s) qui focalise(nt) les neutrons géométriquement sur l'échantillon.

La monochromatisation étant basée sur une diffraction de Bragg (hybride à géométrie directe), la direction du faisceau incident dépend de la longueur d'onde incidente, ce qui nécessite, notemment une base de temps de vol mobile. Ceci alourdit les procédures de changement de configuration et donc limite beaucoup la versatilité de ce type d'instrument. Par ailleurs, le gain important en luminosité, par rapport aux spectromètres à choppers, est obtenu au détriment de la forme de la résolution qui s'apparente plus à une fonction de Voigt (convolution gaussienne-lorentzienne) : la présence d'ailes peut rendre difficile, par exemple, l'analyse de la densité d'états près du pic élastique pour des transferts d'énergie inférieurs à deux ou trois fois la largeur de la résolution ou de signaux quasiélastiques de faible intensité.

19 On parle de Resolution matching. 


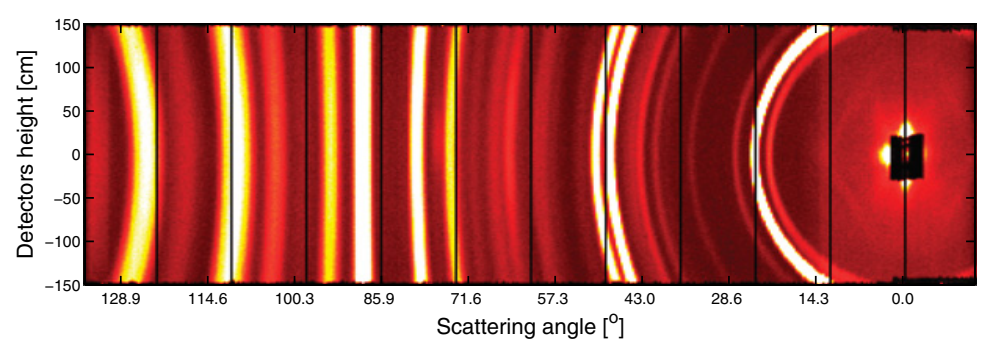

Figure 32. Figure de diffraction de l'osmate de potassium $\left(\mathrm{KOs}_{2} \mathrm{O}_{6}\right)$ sur le détecteur de IN 5 après intégration du temps de vol [40]. La longueur d'onde incidente était de $2.5 \AA$ A. L'axe du faisceau directe est indiqué par le carré du beamstop.

Dans un spectromètre hybride à géométrie directe, la bande de longueur d'onde délivrée par le monochromateur sur un échantillon, dépend des distances guide-monochromateur et monochromateurechantillon. Des distances différentes conduisent à une distribution significative en longueurs d'onde, ce qui permet de travailler dans un mode de focalisation en temps. A l'inverse, on minimise cette distribution si on impose ces distances égales : on parle de focalisation géométrique en mode monochromatique. Ainsi, le choix des distances caractéristiques permet de basculer entre les deux situations extrêmes de distribution en longueur d'onde incidente étroite représentées par les spectromètres à choppers et distribution large pour les spectromètres à focalisation en temps (comparer les courbes de résolution des figures 29 avec celles des figures 15 et 25). Focus, à PSI est l'exemple canonique de ce type de spectromètres, sans doute appelé à se développer dans le futur.

Si les machines à choppers et à focalisation en temps, peuvent être pertinentes sur tous les types de sources (continues ou pulsées), les spectromètres à géométrie inversée sont plus adaptés aux sources à spallation. Dans cette technologie, c'est un faisceau blanc qui illumine l'échantillon, ce qui permet non seulement d'accéder au facteur de structure dynamique $S(Q, \omega)$, mais aussi, et ce simultanément, au facteur de structure $S(Q)$ (spectre de diffraction haute résolution et/ou de diffusion petits angles). La nature du signal détecté est conditionnée par la présence d'un analyseur devant le détecteur (accès à $S(Q, \omega)$ ) ou pas (accès à $S(Q))$. Cette détermination simultanée de la structure et des excitations permet, par exemple, d'attribuer finement une évolution de comportement dynamique à une transition de phase présentant une signature structurale dans la gamme en Q accessible. Cependant, en raison de la difficulté de filtrage des neutrons épithermiques, ces spectromètres souffrent d'un bruit de fond élevé, ce qui est une limitation sévère pour l'étude d'échantillons diffusant peu ou l'utilisation d'environnements échantillons massifs comme, par exemple, des cellules de pression.

Avec les détecteurs à localisation, les spectromètres récents comme IN5 sont aussi des diffractomètres (Fig. 32). Cependant la gamme des neutrons froids incidents ne permet pas d'aller loin en $\mathrm{Q}$ et la résolution spatiale est limitée par la divergence du faisceau qui n'est pas optimisée pour un tel usage.

Une expérience de diffusion inélastique de neutrons réussie résulte de la conjonction de plusieurs éléments :

(i) un problème physique clairement identifié.

(ii) du choix du type de spectromètre en adéquation avec ce problème physique.

(iii) la mesure effectuée sur un échantillon parfaitement caractérisé avant l'expérience (composition chimique et isotopique, structure atomique (diffraction), connaissance des transitions de phases et hystérésis possibles.

(iv) des réglages instrumentaux conduisant, notamment, à des conditions de résolution en énergie adaptées. 
Les comités de sélection des sources sont très vigilants quant aux trois premiers critères. L'ambition de cet article a été de préciser le dernier point, en vue d'alimenter une discussion constructive entre l'expérimentateur et le responsable d'instrument.

La technique de temps de vol se situe à un tournant : jusqu' à présent, cette technique implantée sur réacteurs, i.e. sur source continue, nécessitait de pulser le faisceau, avec pour conséquence une perte très importante du flux utile. Avec l'arrivée de sources à spallation puissantes déjà pulsées à faible fréquence (SNS, JPARK, ESS...), on peut s'attendre dans la décennie à venir, à un saut qualitatif de l'ordre de celui qu'ont connu les techniques de rayons $\mathrm{X}$ avec l'avènement des sources synchrotron de troisième génération.

\section{Remerciements}

Les auteurs tiennent à remercier F. Leclercq-Hugeux, S. Longeville, S. Rols et S. Petit pour les relectures du manuscrit.

\section{Glossaire des abréviations utilisées}

ISIS Isis (nom donné par $M^{m e} \mathrm{M}$. Thatcher lors de l'inauguration en 1984)

RAL Rutherford-Appleton Laboratory

MAPS Massive Array Position sensitive detector Spectrometer

MARI nom de la fille du Pr. Ishikawa (symbole japonais pour vérité)

HET High Energy Transfer

MERLIN -

PRISMA -

LET Low Energy Transfer

IRIS Inelastic high-Resolution tIme-of-flight Spectrometer

KENS Neutron Science Laboratory

MLF Material and Life Science laboratory

PSI Paul Scherrer Institut

SINQ Spallations-NeutronenQuelle

FOCUS FOCUSing time-of-flight spectrometer

MARS Mica Analyzer high-Resolution inverted time-of-flight backscattering Spectrometer

NIST National Institute of Standards and Technology

NCNR NIST Center for Neutron Research

FCS Fermi Chopper Spectrometer

DCS Disk Chopper Spectrometer

ANL Argonne National Laboratory

IPNS Intense Pulse Neutron Source

HRMECS High Resolution Medium Energy Chopper Spectrometer

LRMECS Low Resolution Medium Energy Chopper Spectrometer

QENS Quasi-Elastic Neutron Spectrometer

KEK National Laboratory for High Energy Physics/

High Energy Accelerator Research Organization

J-PARC Japan Proton Accelerator Research Complex

LAM Large Analyser Mica

MAX Multi-Analysers Xtal spectrometer

DNA Biomolecular Dynamics Spectrometer

HRC High Resolution Spectrometer

AMATERA nom japonais du dieu du soleil 


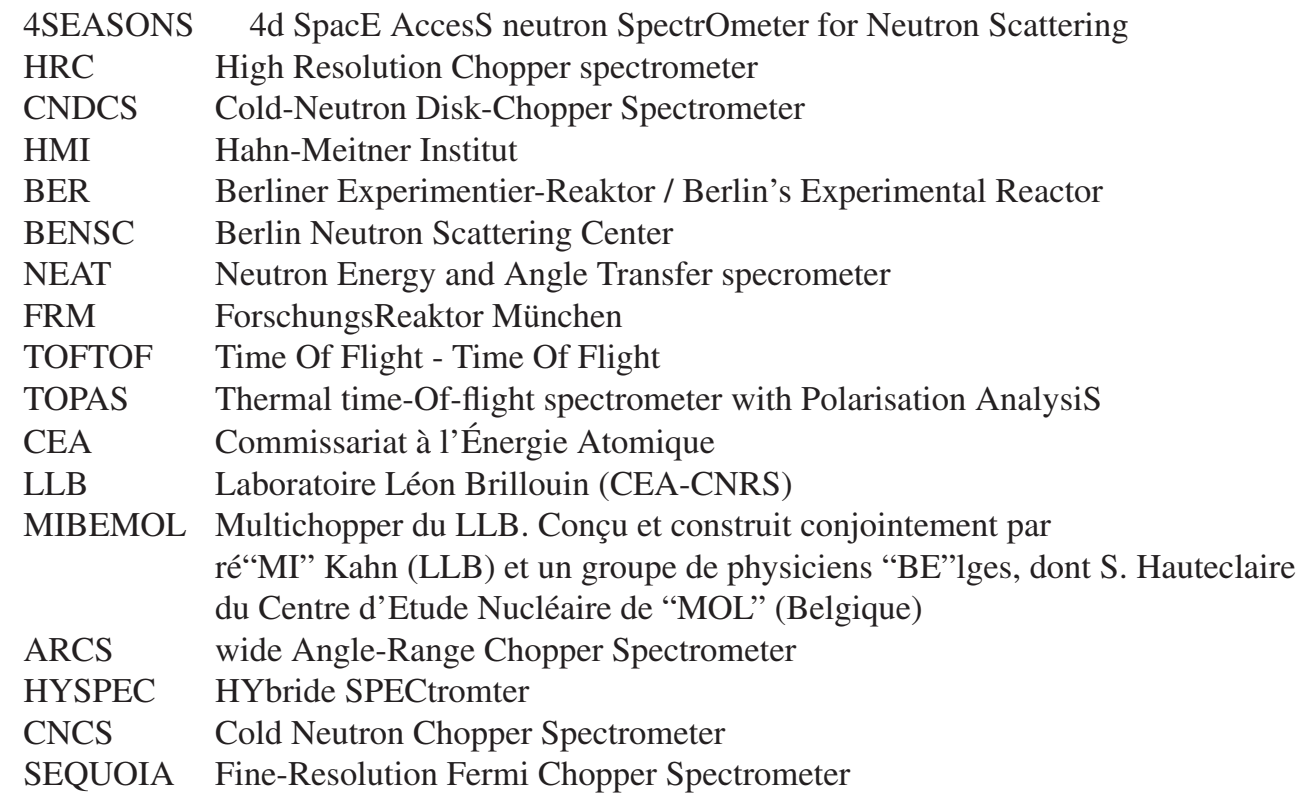

\section{Références}

[1] H. Fizeau, Comp. Rend. Acad. Sci. (Paris), 29(1849)90.

[2] "Expérience de Fizeau" Wikipedia : The Free Encyclopedia. Wikimedia Foundation, Inc. 22 July 2004. Web. 10 juin 2009 〈http ://fr.wikipedia.org/wiki/Experience_de_Fizeau〉

[3] J.R. Dunning, G.B. Pegram, G.A. Fink, D.P. Mitchell \& E. Segre Phys. Rev. 48(1935)704.

[4] L. Passell in Proceedings of the Workshop on Methods for Neutrons Scattering Instrumentation design LBNL-40816 (1996).

[5] E. Fermi, J. Marshall \& L. Marshall, Phys. Rev. 72(1947)193. Un brevet sur le "sélecteur de vitesse" de Fermi fut aussi déposé en 1950 aux États Unis.

[6] Voir par exemple : G. Tagliente and n_ToF Collaboration, Brazilian Journal of Physics, 34(2004)1033.

[7] K. Asai, N. Naoi, T. Iguchi,T. Nishitani, K. Watanabe, \& J. Kawarabayashi, Rev. Sci. Instr. 77(2006)10E721.

[8] T. Perring, Exposé à l'Oxford Neutron School (2007).

[9] P. Ageron, Bulletin d'Informations scientifiques et Techniques du CEA (BIST) janvier 1972.

[10] G. L. Squires, Introduction to the theory of thermal neutron scattering, Cambridge University Press (1978).

[11] R.E. Lechner, F. Volino, A.J. Dianoux, F. Douchin, H. Hervet, G.C. Stirling, ILL Internal Scientific Report 73L8S (1973).

[12] R.E. Lechner, Proceedings of the Workshop on Neutron Instrumentation for SNQ (1984)202.

[13] R.E. Lechner, Proceedings of the International Collaboration on Advanced Neutron Sources ICANS-XI, KEK-Tsukuba (1990)717.

[14] V. Simonet, R. Ballou, résultats non-publiés obtenus sur IN5(2008-2009).

[15] Voir, par exemple : http ://www.jcns.info/src/ws08/presentations/Bernried_Voigt.pdf

[16] R. Lechner, personnal communication.

[17] MD. Lumsden, Jour. Appl. Cryst. 38(2005)405.

[18] F. Rieutord, "INX Programme for Time-of-Flight data reduction" ILL report N 90RI17T (1990).

[19] VF. Sears, Advance Phys. 24(1975)1. 
[20] HH. Paalman, CJ. Pings, Jour. Appl. Phys. 33(1962)2635.

[21] E.O. Wollan and C. G. Shull, Phys.Rev. 73(1948)830.

[22] G.H. Vineyard, Phys. rev. 91(1953)239.

[23] F.G. Bischoff et al. (MSC) Nucl. Sci. Eng. 48(1972)266.

[24] J.R.D. Copley, Computer Phys. Comm. 7(1974)289.

[25] M.W. Johnson, "DISCUS - A Computer Program for the Calculation of Multiple Scattering Effects in Inelastic Neutron Scattering Experiments" AERE-R7682 (1974).

[26] M. Bée, JP. Amoureux, Mol. Phys. 41(1980)287.

[27] R. Scherm, T. Springer, "Proposal of a Multiple Chopper for the Investigation of Very Small Energy Transfert", IFF Jülilch (1967).

[28] R.J. Royston Nucl. Instr. Methods 30(1964)184.

[29] R. Scherm, C. Carlile, A.J. Dianoux, J.B. Suck, J. White, ILL Scientific Report 76S235 (1976).

[30] J. Carpenter, Argonne National Laboratory report $N^{\circ}$ ANL-78-88 (1978).

[31] J. Kalus, B. Dorner, Acta Cryst. A 29(1973)526.

[32] H. Mutka Nucl. Intr. Meth. Phys. Res. A 338(1994)144.

[33] J. Mesot, PSI-SINQ Report Nº95/MJ33-401 (1994).

[34] J. Mesot, S. Jenssen, L. Holitzner, R. Hempelmann, Jour. Neutr, Res. 3(1996)293.

[35] R. Scherm, E. Krüger, Nucl. Instr. Meth. Phys. Res. A 338(1994)1.

[36] C.J. Carlile, D.K. Ross, Jour. Appl. Cryst. 292(1975)292.

[37] S. Janssen, D. Rubio-Temprano, A. Furrer, Physica B 283(200)355.

[38] C.J. Carlile, M.A. Adams, Physica B 182 (1992)431.

[39] P. Allenspach, "Inverted time-of-flight backscattering instrument MARS", PSI-SINQ Technical Report (2000).

[40] H. Mutka, résultats non publiés (2008). 